\title{
Estudo de equações do tipo Navier-Stokes com retardo
}

\section{Sandro Marcos Guzzo}

Orientador : : Eduardo Alex Hernández Morales

Co-orientadora : Gabriela del Valle Planas

Tese apresentada ao Instituto de Ciências Matemáticas e de Computação - ICMC

- USP, como parte dos requisitos para obtenção do título de Doutor em Ciências Área Matemática.

USP - São Carlos

Abril / 2009 

Se $A$ é o sucesso então $A=x+y+z$. $x$ é o trabalho, $y$ é o lazer, e $z$ é manter a boca fechada.

Albert Einstein. 



\section{Agradecimentos}

Ao orientador Professor Dr. Eduardo Hernandez, pela orientação e a colaboração na construção do meu conhecimento. Os ensinamentos do Professor Eduardo me fizeram pensar de forma diferente e interrogativa, contribuindo muito para minha formação como professor e pesquisador.

À co-orientadora Professora Dra. Gabriela Planas, pela orientação e pela paciência. Sua disponibilidade e atenção foram fundamentais, principalmente na redação desta tese.

À minha mulher Sandra Maria Tieppo, pelo apoio e companheirismo. Nesta etapa de cheia de altos e baixos, sua presença tornou-se um forte alicerce.

Aos meus familiares, que acreditaram (e acreditam) em mim, não somente nesta etapa, mas durante toda a minha vida.

Aos professores e amigos da UNIOESTE - Cascavel - Pr, por terem assumido compromissos para que eu pudesse cumprir esta etapa.

Aos amigos do ICMC - USP, alunos ou professores, pela união e ajuda nos momentos de estudo e a descontração nos momentos de lazer.

Aos funcionários do ICMC - USP, pela disposição e empenho. Sua ajuda nos diversos setores como biblioteca, gráfica, secretaria, manutenção, portaria, café, entre outros, não serão esquecidos.

E por fim, mas não menos importante, ao programa PICDT-Capes, pelo apoio financeiro. 



\section{Abstract}

In this work we study the existence of solutions for a Navier-Stokes type equations with delay in the external force and in the nonlinear term. Using the semi-group theory we study the existence of solution for a problem in the form

$$
\begin{aligned}
\frac{d}{d t} u(t) & -\nu \Delta u(t)+\left(F\left(t, u_{t}\right) \cdot \nabla\right) u(t)+\nabla p=g\left(t, u_{t}\right), \quad \text { in } \quad \Omega \times(0, T), \\
\operatorname{div} u(t) & =0, \quad \text { in } \quad \Omega \times(0, T), \\
u(0, x) & =u^{0}(x) \quad x \in \Omega, \\
u(t, x) & =0 \quad t>0, \quad x \in \partial \Omega, \\
u(t, x) & =\phi(t, x) \quad t \in(-\infty, 0), \quad x \in \Omega,
\end{aligned}
$$

where

$$
F\left(t, u_{t}\right)=\int_{-\infty}^{t} \alpha(s-t) u(s) d s+u(t-r)
$$

and

$$
g\left(t, u_{t}\right)=\int_{-\infty}^{t} \beta(s-t) u(s) d s .
$$

On another hand, using the Galerkin approximations method we study the same with $F(\cdot)$ and $g(\cdot)$ given by

$$
F\left(t, u_{t}\right)=u(t-\tau(t)),
$$

and

$$
g\left(t, u_{t}\right)=G(u(t-\rho(t))),
$$

for some $G$ appropriated. In this case, we study also the stability of stationary solutions. 



\section{Resumo}

Neste trabalho estudamos a existência de soluções de equações do tipo Navier-Stokes com retardo na força externa e no termo não linear. Usando a teoria de semigrupos estudamos a existência de soluções para um problema da forma

$$
\begin{aligned}
\frac{d}{d t} u(t) & -\nu \Delta u(t)+\left(F\left(t, u_{t}\right) \cdot \nabla\right) u(t)+\nabla p=g\left(t, u_{t}\right), \quad \text { em } \quad \Omega \times(0, T), \\
\operatorname{div} u(t) & =0, \quad \text { em } \quad \Omega \times(0, T), \\
u(0, x) & =u^{0}(x) \quad x \in \Omega, \\
u(t, x) & =0 \quad t>0, \quad x \in \partial \Omega, \\
u(t, x) & =\phi(t, x) \quad t \in(-\infty, 0), \quad x \in \Omega,
\end{aligned}
$$

onde

$$
F\left(t, u_{t}\right)=\int_{-\infty}^{t} \alpha(s-t) u(s) d s+u(t-r)
$$

e

$$
g\left(t, u_{t}\right)=\int_{-\infty}^{t} \beta(s-t) u(s) d s .
$$

Similarmente, usando a técnica de aproximações de Galerkin, estudamos o problema anterior com $F(\cdot)$ e $g(\cdot)$ dadas por

$$
f\left(t, u_{t}\right)=u(t-\tau(t)),
$$

$\mathrm{e}$

$$
g\left(t, u_{t}\right)=G(u(t-\rho(t))),
$$

para alguma função $G$ apropriada. Neste caso, também estudamos a estabilidade de soluções estacionárias. 



\section{Sumário}

Abstract vii

Resumo ix

Introdução $\quad$ xiii

1 Preliminares 1

1.1 Semigrupos de operadores lineares . . . . . . . . . . . . . . . . . . . . 1

1.2 Potências fracionárias de operadores lineares fechados . . . . . . . . . . . 4

1.3 Espaços de fase abstratos . . . . . . . . . . . . . . . . . . 6

$1.4 \quad \mathrm{O}$ operador de Stokes em $\Omega \subset \mathbb{R}^{n} \ldots \ldots \ldots \ldots \ldots$

2 Soluções em $L^{p}$ para sistemas do tipo Navier-Stokes com memória não $\begin{array}{ll}\text { limitada } & 9\end{array}$

2.1 Existência de soluções para um modelo abstrato . . . . . . . . . . . 9

2.2 Existência de soluções para uma equação de Navier-Stokes com retardamento não limitado . . . . . . . . . . . . . . . . . . . . . . . . . . . . . . . . 18

3 Problema de Navier-Stokes com retardo para dimensão $3 \quad 25$

3.1 Formulação do problema e hipóteses . . . . . . . . . . . . . . . . . 25

3.2 Existência de soluções . . . . . . . . . . . . . . . . . . . . 27

3.3 Estimativa de energia para a solução fraca . . . . . . . . . . . . . . . . 42

3.4 Estabilidade de soluções estacionárias . . . . . . . . . . . . . . . . . . 43

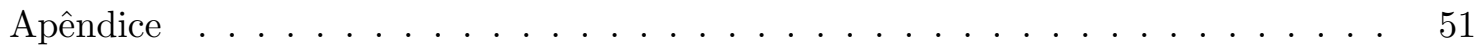

Referências $\quad 59$ 


\section{Introdução}

As equações de Navier-Stokes (NS) são equações que governam o movimento de um fluido como água, óleo, ar, ou plasma, sob condições gerais. Estas equações aparecem no estudo de vários fenômenos importantes e podem ainda ser acopladas a outras equações, como a equação do calor. Para a apresentação das equações de Navier-Stokes, consideremos que um fluido preenche uma região $\Omega \subset \mathbb{R}^{n}$, e que $u=u(t, x)=\left(u_{1}(t, x), \ldots, u_{n}(t, x)\right)$ representa a velocidade, $\rho=\rho(t, x)$ é a densidade e $p=p(t, x)$ é a pressão exercida sobre o fluido, no ponto $x=\left(x_{1}, \ldots, x_{n}\right) \in \Omega$ e no instante $t \in \mathbb{R}$. Então $u, \rho$ e $p$ satisfazem

$$
\begin{aligned}
& \rho\left(\frac{\partial u}{\partial t}+\sum_{i=1}^{n} u_{i} \frac{\partial u}{\partial x_{i}}\right)-\mu \Delta u-(3 \lambda+\mu) \nabla \operatorname{div} u+\nabla p=f \\
& \frac{\partial \rho}{\partial t}+\operatorname{div}(\rho u)=0
\end{aligned}
$$

onde $\mu>0$ é a viscosidade cinemática do fluido, $\nabla=\left(\frac{\partial}{\partial x_{1}}, \ldots, \frac{\partial}{\partial x_{n}}\right)$ é o operador derivada (vetor gradiente) e $f=f(t, x)$ é a força externa agindo sobre o fluido. A segunda equação, a qual é deduzida a partir do princípio da conservação da massa, é conhecida como equação de continuidade. Se o fluido é considerado homogêneo e incompressível, isto é, $\rho$ é uma constante independente de $t$ e $x$, então usualmente é usado que $\rho=1$, e as equações se reduzem a

$$
\begin{aligned}
& \frac{\partial u}{\partial t}-\nu \Delta u+(u \cdot \nabla) u+\nabla p=f \\
& \operatorname{div} u=0
\end{aligned}
$$

onde $(u \cdot \nabla)$ é o operador $\sum_{i=1}^{n} u_{i} \frac{\partial}{\partial x_{i}}$, e $\nu$ é a constante cinemática do fluido. A estas equações, ainda são acrescidas as equações relativas às condições iniciais e de fronteira, dadas por

$$
\begin{aligned}
& u(0, x)=u_{0}(x), \quad x \in \Omega, \\
& u(t, x)=0, \quad t>0, \quad x \in \partial \Omega,
\end{aligned}
$$

onde $\partial \Omega$ é a fronteira de $\Omega$.

Embora as aplicações de interesse pedem $\Omega \subset \mathbb{R}^{3}$, as equações de Navier-Stokes fazem sentido matematicamente para $\Omega \subset \mathbb{R}^{2}$. O estudo de soluções em duas dimensões, 2D, nos 
traz idéias para o tratamento das equações no caso de três dimensões, 3D. Pela mesma razão, o caso linear

$$
\frac{\partial u}{\partial t}-\nu \Delta u+\nabla p=f
$$

também é considerado em alguns estudos.

O modelo físico que leva às equações (NS) é baseado em idéias simples como a lei de conservação de energia e de massa. Por outro lado, sob o ponto de vista matemático, as equações não são simples, uma vez que não são equações lineares. O termo não linear $(u \cdot \nabla) u$ é proveniente de considerações cinemáticas e não das condições físicas do problema. Consequentemente, este termo não pode ser eliminado por remodelamento.

Muitos resultados foram conseguidos a respeito destas equações nos últimos anos, depois dos trabalhos pioneiros de Leray [18] e Hopf [15], que provaram existência de solução fraca para o problema (NS) com $n=3$. Para ser mais preciso, Leray $\left(\Omega=\mathbb{R}^{3}\right)$ e Hopf ( $\Omega$ limitado) provaram que existe $(u, p)$ solução fraca do problema (NS), com as condições iniciais e de fronteira, para $0<t<T$. Todavia, não é conhecido quando esta solução é única.

Para dimensão $n=2$, tem-se existência e unicidade de solução forte para o problema de Navier-Stokes local e global (no tempo). Já para dimensão $n=3$ tem-se existência e unicidade de solução local (no tempo) e para dados iniciais suficientemente pequenos tem-se existência e unicidade global. Podemos citar como referência os trabalhos de Temam [26, 27], Robinson [24], Constantin \& Foias [6], Lions [19].

Duas das principais técnicas usadas para tratar o problema (NS) são o método de aproximações de Galerkin e a teoria de semigrupos.

No método de Galerkin é construída uma solução aproximada para o problema e provase que tal solução aproximada converge para a solução procurada. O método envolve a obtenção de estimativas a priori da solução aproximada. Uma das dificuldades é que tais estimativas são diferentes entre as dimensões 2 e 3 , o que exige, para $n=3$, mais regularidade da solução. Nesta abordagem, citamos os trabalhos de Teman [26, 27] e Constantin \& Foias $[6]$.

O método de semigrupo utiliza estimativas das potências fracionárias do operador de Stokes em espaços $L^{p}$, e o fato de que o semigrupo gerado pelo operador de Stokes é um $C_{0}$-semigrupo analítico. Fato este mostrado por Giga [11]. Antes do trabalho de Giga, este fato era conhecido apenas para espaços de Hilbert. Nesta abordagem, citamos os trabalhos de Bridges [1], Giga [11, 12], Giga \& Miyakawa [13], Cheban \& Duan [7], Rankin [23], Fujita \& Kato [8, 17], Kato [16].

Trabalhos recentes consideram termos com retardo em equações do tipo Navier-Stokes. São considerados vários tipos de retardo, entre limitados, fixos, distribuídos, na força externa e no termo não linear. A motivação para o retardo na força externa, segundo Caraballo $[2,3]$, é que algumas vezes queremos controlar o sistema aplicando uma força que leva em 
consideração não só o estado atual do sistema mas também a sua história. A motivação para o retardo no termo não linear, segundo Liu [20], é que a taxa de variação da velocidade ao seguir o fluido, considerada como a derivada material da velocidade, pode sofrer atraso.

No estudo de equações do tipo de Navier-Stokes com retardo, podemos citar os trabalhos de Caraballo \& Real [3, 2, 4], Garrido-Atienza [10], Taniguchi [25], Planas \& Hernández [22], Liu [20]. Mais precisamente, a equação de Burger, que pode ser considerara como o caso unidimensional, foi estudada por Liu em [20] que a considerou com retardo da forma $u(t-\tau, x) u_{x}(t, x)$ no termo não linear. No caso das equações de Navier-Stokes, Caraballo \& Real [2], estudaram o problema em dimensão $n=2,3$ considerando diferentes tipos de retardo na força externa, e obtiveram existência e unicidade (para $n=2$ ) de solução fraca. Caraballo \& Real [3], obtiveram para $n=2$ a convergência da solução fraca para a solução da equação estacionária, quando $t \rightarrow \infty$. Taniguchi [25] também considerou retardo somente na força externa, e mostrou existência e unicidade de solução fraca global (no tempo), e de solução forte local (no tempo) para $n=3$. Além disso, estudou o comportamento assintótico quando o tempo tende a infinito. Planas \& Hernández [22] estudaram o problema para $n=2$, considerando retardo na força externa e no termo não linear, e provaram a existência de soluções fraca e forte (local no tempo) e a unicidade de solução forte. Também estudaram o comportamento da solução para tempos grandes e a convergência das soluções fracas para a solução da equação estacionária correspondente.

Nesta tese estamos interessados no estudo do problema de Navier-Stokes onde a força externa e o termo não linear contêm termos hereditários. Mais precisamente consideramos o problema,

$$
\begin{aligned}
& \frac{\partial u}{\partial t}-\nu \Delta u+\left(F\left(t, u_{t}\right) \cdot \nabla\right) u+\nabla p=g\left(t, u_{t}\right), \quad t>0, \quad x \in \Omega, \\
& \operatorname{div} u(t, x)=0, \quad t>0, \quad x \in \Omega, \\
& u(t, x)=0, \quad t>0, \quad x \in \partial \Omega, \\
& u(0, x)=u^{0}(x), \quad x \in \Omega, \\
& u(t, x)=\phi(t, x), \quad t \in(-h, 0), \quad x \in \Omega,
\end{aligned}
$$

onde $u_{t}$ representa o retardo $u(t+\theta)$ para $\theta \in(-h, 0), u^{0}$ e $\phi$ são os dados iniciais, $F\left(t, u_{t}\right)$ e $g\left(t, u_{t}\right)$ são expressões com retardo e $h$ pode ser um número positivo finito ou infinito.

Para o estudo deste problema, esta tese está dividida em três capítulos. No primeiro capítulo, consideramos algumas preliminares da teoria básica que será utilizada nos capítulos seguintes. Apresentamos resultados a respeito de semigrupos analíticos, potências fracionárias de um operador, espaços de fase e sobre o operador de Stokes.

No Capítulo 2 estudaremos um problema do tipo (NSR) em dimensão $n$, utilizando abordagem por teoria de semigrupos lineares. O retardo considerado será do tipo distribuído, 
não limitado $(h=\infty)$ e permitirá considerar funções como

$$
F\left(t, u_{t}\right)=\int_{-\infty}^{t} \alpha(s-t) u(s) d s+u(t-r)
$$

e

$$
g\left(t, u_{t}\right)=\int_{-\infty}^{t} \beta(s-t) u(s) d s .
$$

Colocaremos o sistema do tipo Navier-Stokes na forma de um problema abstrato. Após a formulação deste problema abstrato, bem como das hipóteses, mostraremos a existência de (uma única) solução fraca (Teorema 2.1.4) e também estudaremos a regularidade desta solução, (Lemas 2.1.6, 2.1.7 e 2.1.8 e Proposição 2.1.9). Em particular assumindo hipóteses sobre as condições de retardo, mostraremos que esta solução é uma solução clássica (Teorema 2.1.10). Os resultados apresentados neste capítulo estendem os resultados sobre as equações de Navier-Stokes com retardo pois consideram retardos do tipo não limitado.

No Capítulo 3 estudaremos o sistema de Navier-Stokes (NSR) em dimensão 3, utilizando o método de Faedo-Galerkin. O retardo considerado aqui é do tipo pontual e limitado ( $h$ finito):

$$
F\left(t, u_{t}\right)=u_{t}(-\tau(t))=u(t-\tau(t))
$$

e

$$
g\left(t, u_{t}\right)=G(u(t-\rho(t))),
$$

para alguma função $G$ satisfazendo certas condições. Após a formulação adequada do problema, provaremos resultados sobre a existência de solução fraca (Teorema 3.2.1) e forte (Teorema 3.2.2). Como complemento, mostramos que a solução forte define um conjunto absorvente (Corolário 3.2.5). Obtemos uma estimativa de energia para as soluções fracas construídas, e mostraremos que qualquer solução fraca que satisfaz tal estimativa de energia, converge exponencialmente para a solução estacionária quando $t \rightarrow \infty$ (Teorema 3.4.2). Os resultados apresentados neste capítulo estendem o caso bidimensional estudado por Hernández \& Planas em [22] e o caso sem retardo no termo não linear estudado por Caraballo em [2] e em [3]. 


\title{
Capítulo 1
}

\section{Preliminares}

\begin{abstract}
Resumo do capítulo: Neste capítulo introduzimos alguns conceitos, definições e resultados que serão necessários nos capítulos seguintes. Em particular, consideramos algumas questões básicas relativas a teoria de semigrupos lineares, potências fracionárias de operadores lineares fechados e elementos básicos da teoria de equações de Navier-Stokes. Neste capítulo, $(X,\|\cdot\|)$ é um espaço de Banach e para espaços normados $\left(Z,\|\cdot\|_{Z}\right)$ e $\left(W,\|\cdot\|_{W}\right)$ representamos por $\mathcal{L}(Z, W)$ o espaço dos operadores lineares contínuos de $Z$ em $W$ munido da norma de operadores $\|\cdot\|_{\mathcal{L}(Z, W)}$. Quando $Z=W$ escrevemos simplesmente $\mathcal{L}(Z)$ e $\|\cdot\|_{\mathcal{L}(Z)}$
\end{abstract}

\subsection{Semigrupos de operadores lineares}

Nesta seção apresentaremos alguns conceitos e resultados básicos sobre a teoria de semigrupos analíticos de operadores lineares. As demonstrações dos resultados desta seção podem ser encontradas em [21].

Definição 1.1.1. Uma família $(T(t))_{t \geq 0}$ de operadores lineares em $\mathcal{L}(X)$ é um semigrupo de operadores lineares limitados em $X$, se

(i) $T(0)=I$,

(ii) $T(t+s)=T(t) T(s)$, para todos $t, s \geq 0$.

Definição 1.1.2. Um semigrupo de operadores lineares limitados, $(T(t))_{t \geq 0}$, é uniformemente contínuo se $\lim _{t \rightarrow 0^{+}}\|T(t)-I\|_{\mathcal{L}(X)}=0$.

É imediato da definição que se $(T(t))_{t \geq 0}$ é um semigrupo uniformemente contínuo de operadores lineares limitados, então $\lim _{s \rightarrow t}\|T(s)-T(t)\|=0$, para todo $t>0$.

O operador linear $A$ definido por

$$
\begin{aligned}
A: D(A) \subset X & \rightarrow X \\
x & \mapsto A x=\lim _{t \rightarrow 0^{+}} \frac{T(t) x-x}{t}=\left.\frac{d^{+}}{d t} T(t) x\right|_{t=0},
\end{aligned}
$$


sendo,

$$
D(A)=\left\{x \in X ; \quad \lim _{t \rightarrow 0^{+}} \frac{T(t) x-x}{t} \quad \text { existe }\right\},
$$

é chamado de gerador infinitesimal do semigrupo $(T(t))_{t \geq 0}$.

Definição 1.1.3. Um semigrupo de operadores lineares limitados $(T(t))_{t \geq 0}$ em $X$, é um semigrupo fortemente contínuo de operadores lineares limitados se $\lim _{t \rightarrow 0^{+}} T(t) x=x$, para todo $x \in X$. Um semigrupo fortemente contínuo de operadores lineares limitados é dito um semigrupo de classe $C_{0}$ ou simplesmente um $C_{0}$-semigrupo.

Teorema 1.1.4. [21, Teorema 1.2.2] Seja $(T(t))_{t>0}$ um $C_{0}$-semigrupo em $X$. Então existem constantes $\delta \geq 0$ e $M \geq 1$ tais que $\|T(t)\| \leq M e^{\delta t}$ para todo $t \geq 0$.

Se $\delta=0$ então $(T(t))_{t \geq 0}$ é dito uniformemente limitado e se, mais ainda, $M=1$, então $(T(t))_{t \geq 0}$ é chamado um $C_{0}$-semigrupo de contrações.

Teorema 1.1.5. [21, Teorema 1.2.4] Seja $(T(t))_{t \geq 0}$ um $C_{0}$-semigrupo e seja A o seu gerador infinitesimal. Então

(i) $\operatorname{Para} x \in X$,

$$
\lim _{h \rightarrow 0} \frac{1}{h} \int_{t}^{t+h} T(s) x d s=T(t) x
$$

(ii) Para $x \in X, \int_{0}^{t} T(s) x d s \in D(A) e$

$$
A\left(\int_{0}^{t} T(s) x d s\right)=T(t) x-x .
$$

(iii) Para $x \in D(A), T(t) x \in D(A) e$

$$
\frac{d}{d t} T(t) x=A T(t) x=T(t) A x .
$$

(iv) Para $x \in D(A)$

$$
T(t) x-T(s) x=\int_{s}^{t} T(\tau) A x d \tau=\int_{s}^{t} A T(\tau) x d \tau
$$

Vamos agora enunciar o Teorema de Hille-Yosida que caracteriza o gerador infinitesimal de um $C_{0}$-semigrupo de contrações.

Definição 1.1.6. Seja $A: D(A) \subset X \rightarrow X$ um operador linear. $O$ conjunto resolvente de $A$, denotado por $\rho(A)$, é o conjunto formado por todos os números complexos $\lambda$ para os quais $(\lambda I-A)$ é invertível e $(\lambda I-A)^{-1}$ é um operador linear limitado em $X$. A função $R(\cdot): \rho(A) \rightarrow \mathcal{L}(X)$ dada por $R(\lambda: A)=(\lambda I-A)^{-1}$, é chamada de resolvente de $A$. 
Teorema 1.1.7. (Hille-Yosida [21, Teorema 1.3.1]) Um operador linear $A: D(A) \subset X \rightarrow X$ é o gerador infinitesimal de um $C_{0}$-semigrupo de contrações $(T(t))_{t \geq 0}$ em $X$, se e somente se,

(i) A é fechado e $\overline{D(A)}=X$,

(ii) O conjunto resolvente $\rho(A)$ de $A$ contém $\mathbb{R}^{+}$e $\|R(\lambda: A)\| \leq \frac{1}{\lambda}$, para cada $\lambda>0$.

Corolário 1.1.8. [21, Teorema 1.5.3] Um operador linear $A: D(A) \subset X \rightarrow X$ é o gerador infinitesimal de um $C_{0}$-semigrupo $(T(t))_{t \geq 0}$, satisfazendo $\|T(t)\| \leq M e^{\omega t}$, se e somente se,

(i) A é fechado e $D(A)$ é denso em $X$,

(ii) O conjunto resolvente $\rho(A)$ de $A$, contém $(\omega, \infty)$ e $\left\|(R(\lambda: A))^{n}\right\| \leq \frac{M}{(\lambda-\omega)^{n}}$, para todo $\lambda>\omega$ e todo $n \in \mathbb{N}$.

Definição 1.1.9. Seja $(T(t))_{t \geq 0}$ um $C_{0}$-semigrupo em $X$. O semigrupo $(T(t))_{t \geq 0}$ é dito diferenciável para $t>t_{0}$ se, para todo $x \in X$, a função $t \mapsto T(t) x$ é diferenciável para $t>t_{0}$. $O$ semigrupo $(T(t))_{t \geq 0}$ é dito diferenciável, se $(T(t))_{t \geq 0}$ é diferenciável para todo $t>0$.

Definição 1.1.10. Sejam $\varphi_{1}<0<\varphi_{2}, \Delta=\left\{z ; \quad \varphi_{1}<\arg z<\varphi_{2}\right\}$ e $(T(z))_{z \in \Delta}$ uma família de operadores lineares limitados em $X$. A família $(T(z))_{z \in \Delta}$ é chamada semigrupo analítico em $\Delta$ se

(i) $z \mapsto T(z)$ é analítica em $\Delta$,

(ii) $T(0)=I$ e $\lim _{z \rightarrow 0} T(z) x=x$ para todo $x \in X$,

(iii) $T\left(z_{1}+z_{2}\right)=T\left(z_{1}\right) T\left(z_{2}\right)$ para $z_{1}, z_{2} \in \Delta$.

Claramente, a restrição de um semigrupo analítico ao eixo real não negativo é um $C_{0}$-semigrupo.

Teorema 1.1.11. [21, Teorema 2.5.2] Seja $(T(t))_{t \geq 0}$ um $C_{0}$-semigrupo uniformemente limitado, $A: D(A) \subset X \rightarrow X$ seu gerador infinitesimal e suponha que $0 \in \rho(A)$. Então as seguintes condições são equivalentes:

(a) $(T(t))_{t \geq 0}$ pode ser estendido a um semigrupo analítico em algum setor $\Delta_{\delta}=$ $\{z ;|\arg z|<\delta\}$ e $\|T(z)\|$ é uniformemente limitado em cada subsetor fechado $\bar{\Delta}_{\delta^{\prime}}$, $\delta^{\prime}<\delta$, de $\Delta_{\delta}$.

(b) Existe uma constante $C>0$ tal que $\|R(\sigma+i \tau: A)\| \leq \frac{C}{|\tau|}$ para cada $\sigma>0$ e $\tau \neq 0$.

(c) Existe $0<\delta<\frac{\pi}{2}$ e $M>0$ tal que

$$
\rho(A) \supset \Sigma=\left\{\lambda:|\arg \lambda|<\frac{\pi}{2}+\delta\right\} \cup\{0\}
$$

$e\|R(\lambda: A)\| \leq \frac{M}{|\lambda|}$ para todo $\lambda \in \Sigma \backslash\{0\}$.

(d) O semigrupo $(T(t))_{t \geq 0}$ é diferenciável para $t>0$ e existe $C>0$ tal que $\|A T(t)\| \leq \frac{C}{t}$ para todo $t>0$. 
Teorema 1.1.12. Seja $(T(t))_{t \geq 0}$ um $C_{0}$-semigrupo em $X$ tal que $\|T(t)\| \leq M e^{\delta t}$ para todo $t \geq 0$ e seja $A: D(A) \subset X \rightarrow X$ seu gerador infinitesimal. Se $0 \in \rho(A)$ e $(T(t))_{t \geq 0}$ é analítico, então $(T(t))_{t \geq 0}$ é diferenciável para $t>0$ e existe uma constante $C>0$, tal que $\|A T(t)\| \leq \frac{C e^{\delta t}}{t}$ para todo $t>0$.

\subsection{Potências fracionárias de operadores lineares fechados}

Estamos agora interessados em algumas propriedades envolvendo as potências fracionárias de um operador que seja gerador infinitesimal de um semigrupo analítico. As demonstrações dos resultados desta seção podem ser encontrados em [21, Seção 2.6]. Para iniciar, vamos supor que $A: D(A) \subset X \rightarrow X$ é um operador linear fechado e que $X=\overline{D(A)}$. Também assumiremos que

$(\mathbf{H} \rho)$ Existem números positivos $\omega$ e $M$, e uma vizinhança da origem $V$, tal que

$$
\rho(A) \supset \Sigma^{+} \cup V=\Sigma^{+}=\{\lambda ; \quad 0<\omega<|\arg \lambda| \leq \pi\} \cup V,
$$

e $\|R(\lambda: A)\| \leq \frac{M}{1+|\lambda|}$, para todo $\lambda \in \Sigma^{+} \cup V$.

Dos resultados da seção anterior, temos que se $M=1$ e $\omega=\frac{\pi}{2}$, então $-A$ é o gerador infinitesimal de um $C_{0}$ semigrupo. Se $\omega<\frac{\pi}{2}$, então $-A$ é o gerador infinitesimal de um semigrupo analítico.

Definição 1.2.1. Suponha que $A: D(A) \subset X \rightarrow X$ é um operador que satisfaz a hipótese $(H \rho)$ e $\theta \in(\omega, \pi)$. Para $\alpha>0$, definimos o operador

$$
A^{-\alpha}=-\frac{1}{2 \pi i} \int_{\Gamma} \lambda^{-\alpha} R(\lambda: A) d \lambda,
$$

onde $\Gamma$ é o caminho composto das curvas $\rho e^{-i \theta}$, $\rho e^{i \theta}$ e $\rho_{0} e^{i \varphi}$, com $\rho_{0}<\rho<\infty e-\theta \leq \varphi \leq \theta$, $e$ é orientado no sentido do crescimento de $\operatorname{Im} \lambda$.

A integral (1.1) converge para $\alpha>0$, e portanto, define um operador linear limitado em $X$. Além disso, se $\alpha=n(n \in \mathbb{N})$, então o integrando é analítico em $\Sigma^{+}-\{0\}$, e 0 é o único pólo de ordem $n$, do integrando. O teorema dos resíduos pode ser usado então para mostrar que quando $\alpha=n$, a definição acima coincide com a definição clássica de $\left(A^{-1}\right)^{n}$, isto é, $n$ cópias do operador $A^{-1}$.

Se $\omega<\frac{\pi}{2}$ então $-A$ é o gerador infinitesimal de um semigrupo analítico $(T(t))_{t \geq 0}$, e neste caso, podemos ainda obter outra representação para $A^{-\alpha}$.

Definição 1.2.2. Se $-A$ é o gerador infinitesimal de um semigrupo analítico $(T(t))_{t \geq 0}$ em $X$, definimos $A^{0}=I d$ e para $\alpha>0$,

$$
A^{-\alpha}=\frac{1}{\Gamma(\alpha)} \int_{0}^{\infty} t^{\alpha-1} T(t) d t
$$


onde $\Gamma(\cdot)$ é a função Gamma.

A integral em (1.2) converge na topologia do operador para todo $\alpha>0$, e define portanto um operador linear limitado de $X$ em $X$.

Lema 1.2.3. ([21, Lemas 2.6 .3 e 2.6.6]) Para todo $\alpha \geq 0, A^{-\alpha}$ é injetivo. Mais ainda, existe $C>0$ tal que $\left\|A^{-\alpha}\right\| \leq C$ para todo $\alpha \in[0,1]$.

A injetividade de $A^{-\alpha}$ motiva a seguinte definição.

Definição 1.2.4. Assuma que $\omega<\frac{\pi}{2}$. Para cada $\alpha>0$, definimos o operador $A^{\alpha}: R\left(A^{-\alpha}\right) \subset$ $X \rightarrow X$ por $A^{\alpha}=\left(A^{-\alpha}\right)^{-1}$.

Teorema 1.2.5. ([21, Teorema 2.6.8]) Seja $\alpha \geq 0$. As seguintes propriedades são válidas.

(a) $A^{\alpha}$ é um operador fechado com dominio $D\left(A^{\alpha}\right)=R\left(A^{-\alpha}\right)$.

(b) $S e \alpha \geq \beta>0$ então $D\left(A^{\alpha}\right) \subset D\left(A^{\beta}\right)$.

(c) Para cada $\alpha \geq 0, X=\overline{D\left(A^{\alpha}\right)}$.

(d) Se $\alpha, \beta \in \mathbb{R}$, então $A^{\alpha+\beta} x=A^{\alpha} A^{\beta} x$, para todo $x \in D\left(A^{\gamma}\right)$ com $\gamma=\max \{\alpha, \beta, \alpha+$ $\beta\}$.

Teorema 1.2.6. ([21, Teorema 2.6.13]) As seguintes propriedades são verificadas.

(a) $T(t) x \in D\left(A^{\alpha}\right)$ para todo $t>0$ e todo $\alpha \geq 0$.

(b) Para cada $x \in D\left(A^{\alpha}\right)$, temos que $T(t) A^{\alpha} x=A^{\alpha} T(t) x$.

(c) Para cada $t>0$, e todo $\alpha \geq 0$, o operador $A^{\alpha} T(t)$ é limitado e existem $M_{\alpha}>0$ e $\delta>0$ tais que $\left\|A^{\alpha} T(t)\right\|_{\mathcal{L}(X)} \leq \frac{M_{\alpha}}{t^{\alpha}} e^{-\delta t}$ para todo $t>0$.

(d) Para cada $\alpha \in[0,1]$, existe $C_{\alpha}>0$ tal que $\|T(t) x-x\| \leq C_{\alpha} t^{\alpha}\left\|A^{\alpha} x\right\|$, para cada $x \in D\left(A^{\alpha}\right)$ e todo $t>0$.

Enunciaremos agora alguns resultados sobre a existência e regularidade de soluções do problema de valor inicial

$$
\left\{\begin{array}{l}
u^{\prime}(t)-A u(t)=f(t) \quad t \in[0, a], \\
u(0)=u_{0},
\end{array}\right.
$$

onde $A: D(A) \subset X \rightarrow X$ é o gerador infinitesimal de um $C_{0}$-semigrupo $(T(t))_{t \geq 0}$ em $X$ e $f:[0, a] \rightarrow X$ é uma função.

Definição 1.2.7. Uma função $u:[0, a] \rightarrow X$ é chamada solução clássica do problema (1.3) se $u(\cdot)$ é contínua em $[0, a]$, continuamente diferenciável em $(0, a], u(t) \in D(A)$ para $0<t<a$ e u(t) satisfaz (1.3) em $[0, a]$.

Definição 1.2.8. Uma função $u:[0, a] \rightarrow X$ é chamada solução forte do problema de valor inicial (1.3) se $u(\cdot)$ é diferenciável para quase todo $t \in[0, a], u^{\prime}(t) \in L^{1}(0, a ; X), u(0)=u_{0} e$ $u^{\prime}(t)-A u(t)=f(t)$ para quase todo $t \in[0, a]$. 
Se $u(\cdot)$ é uma solução clássica de (1.3) então

$$
u(t)=T(t) u_{0}+\int_{0}^{t} T(t-s) f(s) d s, \quad t \in[0, a] .
$$

Isto motiva a próxima definição.

Definição 1.2.9. Uma função $u \in C([0, a]: X)$ é chamada de solução fraca do problema de valor inicial (1.3) se $u(\cdot)$ verifica $(1.4)$ em $[0, a]$.

Nos próximos resultados são consideradas algumas condições sob as quais uma solução fraca de (1.3) é de fato uma solução clássica ou uma solução forte.

Teorema 1.2.10. ([21, Corolário 4.2.5]) Seja A é o gerador infinitesimal de um $C_{0}$-semigrupo $(T(t))_{t \geq 0}$ em $X$. Se $f \in L^{1}(0, a ; X)$ é continuamente diferenciável em $[0, a]$ e $u_{0} \in D(A)$, então a solução fraca de (1.3) em [0,a) é uma solução clássica.

Teorema 1.2.11. ([21, Corolário 4.2.6]) Seja A é o gerador infinitesimal de um $C_{0}$-semigrupo $(T(t))_{t \geq 0}$ em $X$ e $f \in L^{1}(0, a ; X) \cap C((0, a]: X)$. Se $f(s) \in D(A)$ para todo $s \in(0, a)$, $A f(s) \in L^{1}(0, a ; X)$ e $u_{0} \in D(A)$, então a solução fraca de (1.3) é uma solução clássica de (1.3) em $[0, a]$.

Teorema 1.2.12. ([21, Corolário 4.2.10]) Seja $A$ é o gerador infinitesimal de um $C_{0}$ semigrupo $(T(t))_{t \geq 0}$. Se $f:[0, a] \rightarrow X$ é diferenciável quase sempre em $[0, a], f^{\prime} \in L^{1}(0, a ; X)$ e $u_{0} \in D(A)$, então a solução fraca de (1.3) é uma solução forte de (1.3) em [0,a].

Teorema 1.2.13. ([21, Corolário 4.2.11]) Seja $A$ é o gerador infinitesimal de um $C_{0^{-}}$ semigrupo $(T(t))_{t \geq 0}$ e suponha que $X$ é reflexivo. Se $f:[0, a] \rightarrow X$ é Lipschitz e $u_{0} \in D(A)$, então a solução fraca de (1.3) é uma solução forte de (1.3) em [0,a].

Teorema 1.2.14. ([21, Teorema 4.3.1]) Sejam A o gerador infinitesimal de um semigrupo analítico $(T(t))_{t \geq 0}$ e $f \in L^{p}(0, a ; X)$ com $1<p<\infty$. Se u(.) é a solução fraca de (1.3) em $[0, a]$ então $u \in C^{\frac{p-1}{p}}([\epsilon, a]: X)$ para todo $\epsilon>0$. Se além disso, $u_{0} \in D(A)$ então $u \in C^{\frac{p-1}{p}}([0, a]: X)$.

Teorema 1.2.15. ([21, Corolário 4.3.3]) Seja A é o gerador infinitesimal de um semigrupo analítico $(T(t))_{t \geq 0}$ em $X$. Se $f \in L^{1}(0, a ; X)$ é localmente Hölder contínua $(0, a]$, então para cada $u_{0} \in X$ o problema de valor inicial (1.3) possui uma única solução clássica em $[0, a]$.

\subsection{Espaços de fase abstratos}

Seja $\left(Z,\|\cdot\|_{Z}\right)$ um espaço de Banach. Neste trabalho, a notação $\mathcal{B}_{Z}$ (ou simplesmente $\mathcal{B}$ quando não houver possibilidade de confusão) representa um espaço linear formado por funções definidas de $(-\infty, 0]$ em $Z$ munido de uma seminorma $\|\cdot\|_{\mathcal{B}_{Z}}$ que verifica os seguintes axiomas. 
(A) Se $x:(-\infty, \sigma+b) \rightarrow Z, b>0, \sigma>0$, é contínua em $[\sigma, \sigma+b]$ e $x_{\sigma} \in \mathcal{B}_{Z}$, então para cada $t \in[\sigma, \sigma+b]$, as seguintes propriedades são verificadas

(a) $x_{t} \in \mathcal{B}_{Z}$,

(b) $\|x(t)\| \leq H_{Z}\left\|x_{t}\right\|_{\mathcal{B}_{Z}}$,

(c) $\left\|x_{t}\right\|_{\mathcal{B}_{Z}} \leq K_{Z}(t-\sigma) \sup _{s \in[\sigma, t]}\|x(s)\|_{Z}+M_{Z}(t-\sigma)\left\|x_{\sigma}\right\|_{\mathcal{B}_{Z}}$,

onde $H_{Z}>0$ é uma constante, $K_{Z}, M_{Z}:[0, \infty) \rightarrow[1, \infty), K_{Z}(\cdot)$ é contínua, $M_{Z}(\cdot)$ é localmente limitada e $H_{Z}, K_{Z}, M_{Z}$ são independentes de $x(\cdot), \sigma, b$ e $t \in[\sigma, \sigma+b]$.

(A1) Para a função $x(\cdot)$ em $(\mathbf{A})$, a função $t \mapsto x_{t}$ é contínua de $[0, \sigma+b)$ em $\mathcal{B}_{Z}$.

(B) O espaço $\mathcal{B}_{Z}$ é completo.

(C2) Se $\left(\varphi^{n}\right)_{n \in \mathbb{N}}$ é uma sequência uniformemente limitada em $C((-\infty, 0]: Z)$ formada por funções com suporte compacto e $\varphi^{n} \rightarrow \varphi$ na topologia compacto aberta, então $\varphi \in \mathcal{B}_{Z}$ e $\left\|\varphi^{n}-\varphi\right\|_{\mathcal{B}_{Z}} \rightarrow 0$ quando $n \rightarrow \infty$

Consideremos agora um exemplo de espaço de fase.

$\mathbf{O}$ espaço $\mathbf{C}_{\mathbf{r}} \times \mathbf{L}^{\mu}(\mathbf{g}, \mathbf{X}), \mathbf{r}>\mathbf{0}, \mu \geq \mathbf{1}$. O espaço $\mathcal{B}=C_{r} \times L^{\mu}(g, X)$, consiste das classes de funções $\phi:(-\infty, 0] \rightarrow X$ tais que $\phi(\cdot)$ é contínua em $[-r, 0]$, é mensurável no sentido de Lebesgue, e $g(\cdot)\|\phi(\cdot)\|^{\mu}$ é Lebesgue integrável sobre $(-\infty,-r)$. O espaço $\mathcal{B}$ é munido da seminorma definida por

$$
\|\phi\|_{\mathcal{B}}:=\sup _{-r \leq s \leq 0}\|\phi(s)\|_{X}+\left(\int_{-\infty}^{-r} g(s)\|\phi(s)\|^{\mu} d s\right)^{\frac{1}{\mu}} .
$$

O espaço $\mathcal{B}=C_{r} \times L^{\mu}(g, X)$ satisfaz os axiomas (A), (A-1), e (B), quando $g(\cdot)$ satisfaz as condições (g-5) e (g-6) seguintes.

(g-1) A aplicação $t \mapsto \sup \left\{\frac{g(s+t)}{g(s)} ; \quad-\infty<s \leq-t\right\}$ é localmente limitada para $t \geq 0$.

$(\mathrm{g}-2) \quad g(s) \rightarrow \infty$ quando $s \rightarrow-\infty$.

$(\mathrm{g}-3) a(t)=\lim _{s \rightarrow-\infty} \frac{g(s+t)}{g(s)}$ existe para cada $t$ e $a(t)$ é contínua em $t$.

(g-4) $\ln (g(s))$ é uniformemente contínua em $(-\infty, 0]$.

(g-5) $g(\cdot)$ é localmente integrável em $(-\infty,-r)$, isto é, $\int_{\theta}^{-r} g(s) d s<\infty$ para qualquer $\theta \in$ $(-\infty,-r)$

(g-6) Existe uma função $\gamma(\cdot)$, não negativa, localmente limitada em $(-\infty, 0]$ tal que $g(\xi+s) \leq$ $\gamma(\xi) g(s)$, para todo $\xi \leq 0$ e $\theta \in(-\infty,-r) \backslash N_{\xi}$, onde $N_{\xi} \subset(-\infty,-r)$ é um conjunto com medida nula. 
No caso particular onde $r=0$ e $\mu=2$, podemos tomar $H=1, M(t)=\gamma(-t)^{1 / 2}$ e $K(t)=$ $1+\left(\int_{-t}^{0} g(s) d s\right)^{\frac{1}{2}}$ para $t \geq 0$. Para maiores detalhes sobre a teoria de espaços de fase, citamos Hino [14].

Para finalizar nossas observações, sobre espaços de fase, observamos que se $(T(t))_{t \geq 0}$ é um $C_{0}$-semigrupo em $Z$ e $(W(t))_{t \geq 0}$ é a família de operadores definida por

$$
(W(t) \phi)(\theta):= \begin{cases}T(t+\theta) \phi(0), & -t \leq \theta \leq 0, \\ \phi(t+\theta), & -\infty<\theta<-t,\end{cases}
$$

então $(W(t))_{t \geq 0}$ é um $C_{0}$-semigrupo de operadores lineares limitados em $\mathcal{B}_{Z}$.

\subsection{O operador de Stokes em $\Omega \subset \mathbb{R}^{n}$}

Agora fazemos alguns breves comentários sobre o operador de Stokes. Seja $\Omega \subset \mathbb{R}^{n}$ um aberto limitado com fronteira $\partial \Omega$ de classe $C^{2}$, e seja $\nu(x)$ o vetor normal externo a $\Omega$ em $x \in \partial \Omega$. Definamos

$$
\begin{gathered}
\mathcal{V}=\left\{u \in\left(C_{0}^{\infty}(\Omega)\right)^{n} ; \quad \operatorname{div} u=0\right\} \\
C_{\sigma}^{\infty}(\Omega)=\left\{u \in\left(L^{2}(\Omega)\right)^{n} ; \quad \operatorname{div} u=0 \quad \text { em } \quad \Omega, \quad u \cdot \nu=0 \quad \text { em } \quad \partial \Omega\right\} .
\end{gathered}
$$

Sejam $H$ e $V$ os espaços definidos por

$$
H=\overline{\mathcal{V}}^{\left(L^{2}(\Omega)\right)^{n}} \quad \text { e } \quad V=\overline{\mathcal{V}}^{\left(H_{0}^{1}(\Omega)\right)^{n}} .
$$

É claro que $H$ munido do produto interno usual em $\left(L^{2}(\Omega)\right)^{n},\langle u, v\rangle=\sum_{i=1}^{n}\left\langle u_{i}, v_{i}\right\rangle_{L^{2}(\Omega)}$, é um espaço de Hilbert. Seja $P$ a projeção de $\left(L^{2}(\Omega)\right)^{n}$ em $H$ e $D(A)=\left(H^{2}(\Omega)\right)^{n} \cap\left(H_{0}^{1}(\Omega)\right)^{n} \cap H$. O Operador de Stokes em $\Omega \subset \mathbb{R}^{n}$ é o operador $A: D(A) \subseteq H \rightarrow H$ definido por

$$
A u=-P \Delta u=-P\left(\Delta u_{1}, \Delta u_{2}, \ldots, \Delta u_{n}\right)
$$

De Cattabriga [5] conhecemos o seguinte resultado.

Teorema 1.4.1. O operador de Stokes $A=-P \Delta$ é o gerador de um semigrupo analítico em $H$. 


\title{
Capítulo 2
}

\section{Soluções em $L^{p}$ para sistemas do tipo Navier-Stokes com memória não limitada}

\author{
Resumo do capítulo: Neste capítulo estudamos a existência de soluções para \\ uma classe de sistemas do tipo de Navier-Stokes com retardo não-limitado. Para obter \\ nossos resultados faremos uso da teoria de semigrupos analíticos em espaços de Banach e \\ assumiremos que o espaço de fase (o espaço das histórias) é definido de maneira axiomática.
}

\subsection{Existência de soluções para um modelo abstrato}

Neste capítulo estudamos a existência de soluções para uma classe de sistemas abstratos com retardamento não limitado descritos na forma

$$
\begin{aligned}
u^{\prime}(t) & =A u(t)+F\left(t, u_{t}, u(t)\right)+g\left(t, u_{t}\right), \quad t \in[0, a], \\
u_{0} & =\varphi \in \mathcal{B},
\end{aligned}
$$

onde $A$ é o gerador de um semigrupo analítico $(T(t))_{t \geq 0}$ definido sobre um espaço de Banach $(X,\|\cdot\|)$, a história $u_{t}:(-\infty, 0] \rightarrow X, u_{t}(\theta)=u(t+\theta)$, pertence a um espaço de Banach $\mathcal{B}$ definido de maneira axiomática e $F:[0, a] \times \mathcal{B} \times X \rightarrow X, g:[0, a] \times \mathcal{B} \rightarrow X$ são funções apropriadas.

O estudo da solubilidade do sistema (2.1)-(2.2) tem por finalidade o estudo da existência de soluções clássicas para sistemas de tipo Navier-Stokes com retardamento não limitado. Uma classe particular de sistemas sobre os quais será possível aplicar a teoria desenvolvida, podem ser descritos na forma 


\section{Capítulo 2. Soluções em $L^{p}$ para sistemas do tipo Navier-Stokes com memória

$$
\begin{aligned}
& u^{\prime}(t, \xi)=\nu \Delta u(t, \xi)-\left(\left(\int_{-\infty}^{t} \alpha(s-t) u(s, \xi) d s+u(t-r, \xi)\right) \cdot \nabla\right) u(t, \xi) \\
& \quad+\nabla p(t, \xi)+\int_{-\infty}^{t} \beta(s-t) u(s, \xi) d s, \quad(t, \xi) \in[0, a] \times \Omega, \\
& \operatorname{div} u(t)=\sum_{i=1}^{n} \frac{\partial}{\partial \xi_{i}} u(t, \xi)=0, \quad(t, \xi) \in[0, a] \times \Omega, \\
& u(t, \xi)=0, \quad(t, \xi) \in[0, a] \times \partial \Omega, \\
& u(t, \xi)=\varphi(t, \xi), \quad(t, \xi) \in(-\infty, 0] \times \Omega .
\end{aligned}
$$

Introduzimos agora algumas notações e o esquema técnico geral usado neste capítulo. No que segue deste capítulo, $(X,\|\cdot\|)$ e $\left(Y,\|\cdot\|_{Y}\right)$ são espaços de Banach, $Y \subset X$ e assumiremos que a inclusão $i:\left(Y,\|\cdot\|_{Y}\right) \rightarrow(X,\|\cdot\|)$ é contínua. Adotaremos também as notações introduzidas no Capítulo 1. O conjunto $B_{r}\left(z_{0}, Z\right)$ é a bola de raio $r$ e centro $z_{0}$ de $Z$. Em particular, $\mathcal{B}_{X}$ e $\mathcal{B}_{Y}$ serão espaços de fase como aqueles introduzidos na seção 1.3, e nós sempre assumiremos que $\mathcal{B}_{Y} \subset \mathcal{B}_{X}$ e que a inclusão de $\mathcal{B}_{Y}$ em $\mathcal{B}_{X}$ é contínua. Além do anterior, para $Z=X$ ou $X=Y$, definimos as constantes $K_{a, Z}=\sup _{s \in[0, a]} K_{Z}(s)$ e $M_{a, Z}=\sup _{s \in[0, a]} M_{Z}(s)$, onde $K_{Z}(\cdot)$ e $M_{Z}(\cdot)$ são as funções nos axiomas de espaço de fase.

Para espaços de Banach $\left(Z,\|\cdot\|_{Z}\right)$ e $\left(W,\|\cdot\|_{W}\right)$ usaremos a notação $\mathcal{L}(Z, W)$ para o espaço dos operadores lineares contínuos de $Z$ em $W$ munido da topologia da convergência uniforme e escreveremos simplesmente $\mathcal{L}(Z)$ quando $Z=W$. A notação $Z \hookrightarrow W$ indica que $Z \subset W$ e que a inclusão $i_{c}: Z \rightarrow W$ é contínua. Finalmente para um operador fechado $S: D(S) \subset Z \rightarrow W$, denotaremos por $[S]$ o espaço $D(S)$ munido da norma do gráfico, $\|x\|_{[S]}=\|x\|+\|S x\|, x \in D(S)$.

No que segue do capítulo sempre assumiremos que as seguintes propriedades são satisfeitas.

(i) A é o gerador infinitesimal de um semigrupo analítico $(T(t))_{t \geq 0}$ em $X$. O semigrupo $(T(t))_{t \geq 0}$ é um $C_{0}$-semigrupo em $Y$, e $T(\cdot) x \in C((0, \infty), Y)$ para cada $x \in X$.

(ii) Existe $r>0$ tal que $(-A)^{\alpha} T(\cdot) \in C((0, \infty) ; \mathcal{L}(X, Y))$ e $D\left((-A)^{\alpha}\right) \hookrightarrow Y$ para todo $\alpha \in\left[\frac{1}{2}, \frac{1}{2}+r\right]$. Para todo $\beta>0$ e $\alpha \in\left[\frac{1}{2}, \frac{1}{2}+r\right]$ existem constantes positivas $C_{\beta}, D_{\alpha}$, e $\sigma(\alpha)=\sigma(\alpha, X, Y)<1-\alpha$ tais que

$$
\left\|(-A)^{\beta} T(t)\right\|_{\mathcal{L}(X)} \leq \frac{C_{\beta}}{t^{\beta}} \quad \text { e } \quad\left\|(-A)^{\alpha} T(t)\right\|_{\mathcal{L}(X, Y)} \leq \frac{D_{\alpha}}{t^{\sigma(\alpha)+\alpha}}
$$

para todo $t \in(0, a]$.

(iii) Existe uma função $\widetilde{F}:[0, a] \times \mathcal{B}_{Y} \times Y \rightarrow X$ tal que $F(s, \psi, w)=(-A)^{\frac{1}{2}} \widetilde{F}(s, \psi, w)$ para todo $(s, \psi, w) \in[0, a] \times \mathcal{B}_{Y} \times Y$. Existem funções crescentes $L_{\widetilde{F}}^{i} \in C([0, \infty),[0, \infty))$, 
$i=0,1,2$, e $\gamma_{1} \in(0,1)$ tais que

$$
\begin{aligned}
\| \widetilde{F}\left(t, \psi_{1}, w_{1}\right) & -\widetilde{F}\left(s, \psi_{2}, w_{2}\right) \| \\
& \leq L_{\widetilde{F}}^{0}(R)|t-s|^{\gamma_{1}}+L_{\widetilde{F}}^{1}(R)\left\|\psi_{1}-\psi_{2}\right\|_{\mathcal{B}_{Y}}+L_{\widetilde{F}}^{2}(R)\left\|w_{1}-w_{2}\right\|_{Y},
\end{aligned}
$$

para todo $\left(\psi_{i}, w_{i}\right) \in B_{R}\left(0, \mathcal{B}_{Y}\right) \times B_{R}(0, Y)$ e todo $t, s \in[0, a]$.

(iv) A função $g(\cdot)$ é contínua de $[0, a] \times \mathcal{B}_{Y}$ em $X$ e existe uma função crescente $L_{g} \in$ $C([0, \infty),(0, \infty))$ e $\gamma_{2} \in(0,1)$ tal que

$$
\left\|g\left(t, \varphi_{1}\right)-g\left(s, \varphi_{2}\right)\right\| \leq L_{g}(R)\left(|t-s|^{\gamma_{2}}+\left\|\varphi_{1}-\varphi_{2}\right\|_{\mathcal{B}_{Y}}\right)
$$

para todo $\varphi_{i} \in B_{R}\left(0, \mathcal{B}_{Y}\right)$ e todo $t, s \in[0, a]$.

Introduzimos agora os seguintes conceitos de solução para o sistema (2.1)-(2.2).

Definição 2.1.1. Uma função $u:(-\infty, b] \rightarrow Y, 0<b \leq a$, é chamada de solução $Y$ fraca do problema abstrato (2.1)-(2.2) em $[0, b]$, se $u_{0}=\varphi \in \mathcal{B}_{Y}, u \in C([0, b] ; Y)$, a função $s \mapsto(-A)^{\frac{1}{2}} T(t-s)\left[(-A)^{-\frac{1}{2}} g\left(s, u_{s}\right)+\widetilde{F}\left(s, u_{s}, u(s)\right)\right]$ pertence a $L^{1}([0, t] ; Y)$ e

$$
u(t)=T(t) \varphi(0)+\int_{0}^{t}(-A)^{\frac{1}{2}} T(t-s)\left[(-A)^{-\frac{1}{2}} g\left(s, u_{s}\right)+\widetilde{F}\left(s, u_{s}, u(s)\right)\right] d s, \quad t \in[0, b] .
$$

Definição 2.1.2. Uma função $u:(-\infty, b] \rightarrow X, 0<b \leq a$, é chamada de solução fraca do problema de Cauchy abstrato (2.1)-(2.2) se $u_{0}=\varphi$ e

$$
u(t)=T(t) \varphi(0)+\int_{0}^{t} T(t-s)\left[g\left(s, u_{s}\right)+F\left(s, u_{s}, u(s)\right)\right] d s, \quad t \in[0, b] .
$$

Definição 2.1.3. Uma função $u:(-\infty, b] \rightarrow X, 0<b \leq a$, é chamada de solução clássica de (2.1)-(2.2) em $[0, b]$, se $u_{0}=\varphi, u \in C^{1}((0, a] ; X) \cap C((0, a] ;[D(A)])$ e (2.1) é satisfeita para cada $t \in(0, b]$.

Estamos agora em condições de estabelecer nosso primeiro resultado sobre existência de soluções.

Teorema 2.1.4. Para cada $\varphi \in \mathcal{B}_{Y}$ existe $b \in(0, a]$ e uma única solução $Y$-fraca de (2.1)(2.2) em $[0, b]$.

Prova. Das propriedades das funções $g(\cdot)$ e $\widetilde{F}(\cdot)$, podemos escolher números positivos $R$, $C(R)$ tais que $\|\widetilde{F}(t, \psi, x)\| \leq C(R)$ e $\left\|(-A)^{-\frac{1}{2}} g(t, \psi)\right\| \leq C(R)$ para todo $t \in[0, R]$ e todo $(\psi, x) \in B_{R_{1}}\left(0, \mathcal{B}_{Y}\right) \times B_{R_{1}}(0, X) \operatorname{com} R_{1}=K_{a, Y} R+\left(K_{a, Y} H_{Y}+M_{a, Y}\right)\|\varphi\|_{\mathcal{B}_{Y}}$. Fixemos agora $0<b \leq a$ tal que

$$
\sup _{s \in[0, b]}\|T(t) \varphi(0)-\varphi(0)\|_{Y}+\frac{4 C(R) D_{\frac{1}{2}}}{1-2 \sigma\left(\frac{1}{2}\right)} b^{\frac{1}{2}-\sigma\left(\frac{1}{2}\right)}<R,
$$




$$
K_{a, Y}\left(\left\|(-A)^{-\frac{1}{2}}\right\|_{\mathcal{L}(X)} L_{g}\left(R_{1}\right)+L_{F}^{1}\left(R_{1}\right)+L_{F}^{2}\left(R_{1}\right)\right) \frac{2 D_{\frac{1}{2}}}{1-2 \sigma\left(\frac{1}{2}\right)} b^{\frac{1}{2}-\sigma\left(\frac{1}{2}\right)}<1 .
$$

Seja $\mathcal{F}$ o espaço $\mathcal{F}=\left\{u:(-\infty, b] \rightarrow Y ; u \in C([0, b], Y), u_{0}=\varphi\right\}$ munido da métrica $d(u, v)=\sup _{t \in[0, a]}\|u(t)-v(t)\|_{Y}$, e o sub-espaço

$$
\mathcal{F}_{R}=\left\{u \in \mathcal{F}: \quad \sup _{t \in[0, a]}\|u(t)-\varphi(0)\|_{Y} \leq R\right\} .
$$

Sobre o espaço $\mathcal{F}_{R}$ definimos a função $\mathcal{T}: \mathcal{F}_{R} \rightarrow \mathcal{F}_{R}$ por $(\mathcal{T} u)_{0}=\varphi$ e

$$
\mathcal{T} u(t)=T(t) \varphi(0)+\int_{0}^{t}(-A)^{\frac{1}{2}} T(t-s)\left[(-A)^{-\frac{1}{2}} g\left(s, u_{s}\right)-\widetilde{F}\left(s, u_{s}, u(s)\right)\right] d s, \quad t \in[0, b] .
$$

No que segue mostraremos que $\mathcal{T}$ é uma contração em $\mathcal{F}_{R}$, e como conseqüência, que existe uma única solução $Y$-fraca de (2.1)-(2.2) em $[0, b]$. Para começar, mostraremos que $\mathcal{T} u \in \mathcal{F}$ para cada $u \in \mathcal{F}_{R}$. Seja $u \in \mathcal{F}_{R}$. Fazendo uso dos axiomas do espaço $\mathcal{B}_{Y}$ vemos que para $s \in[0, b]$,

$$
\begin{aligned}
\left\|u_{s}\right\|_{\mathcal{B}_{Y}} & \leq K_{a, Y} \sup _{0 \leq \theta \leq s}\|u(\theta)\|_{Y}+M_{a, Y}\|\varphi\|_{\mathcal{B}_{Y}} \\
& \leq K_{a, Y} \sup _{0 \leq \theta \leq s}\|u(\theta)-\varphi(0)\|_{Y}+K_{a, Y}\|\varphi(0)\|_{Y}+M_{a, Y}\|\varphi\|_{\mathcal{B}_{Y}} \\
& \leq K_{a, Y} R+K_{a, Y} H_{Y}\|\varphi\|_{\mathcal{B}_{Y}}+M_{a, Y}\|\varphi\|_{\mathcal{B}_{Y}} \\
& \leq R
\end{aligned}
$$

de onde obtemos que $\left\|\widetilde{F}\left(s, u_{s}, u(s)\right)\right\|_{Y} \leq C(R)$ e $\left.\|(-A)^{-\frac{1}{2}} g\left(s, u_{s}\right)\right) \|_{Y} \leq C(R)$ para todo $s \in[0, b]$. Para reduzir as notações, $G:[0, b] \rightarrow Y$ será a função definida por $G(s)=(-A)^{-\frac{1}{2}} g\left(s, u_{s}\right)+\widetilde{F}\left(s, u_{s}, u(s)\right)$. Veja que $\|G(s)\| \leq 2 C(R)$ para todo $s \in[0, b]$. Das observações anteriores, para $t \in[0, b)$ e $h>0$ tais que $t+h \in[0, b]$, vemos que

$$
\begin{aligned}
& \|\mathcal{T} u(t+h)-\mathcal{T} u(t)\|_{Y} \\
& \leq\|T(t+h) \varphi(0)-T(t) \varphi(0)\|_{Y} \\
& +\int_{t}^{t+h}\left\|(-A)^{\frac{1}{2}} T(t+h-s)\right\|_{\mathcal{L}(X, Y)}\left\|(-A)^{-\frac{1}{2}} g\left(s, u_{s}\right)+\widetilde{F}\left(s, u_{s}, u(s)\right)\right\| d s \\
& +\int_{0}^{t}\left\|(-A)^{\frac{1}{2}} T(t-s)(T(h)-I)\left[(-A)^{-\frac{1}{2}} g\left(s, u_{s}\right)+\widetilde{F}\left(s, u_{s}, u(s)\right)\right]\right\|_{Y} d s \\
& \leq\|T(t+h) \varphi(0)-T(t) \varphi(0)\|_{Y}+2 C(R) \int_{t}^{t+h} \frac{D_{\frac{1}{2}}}{(t+h-s)^{\frac{1}{2}+\sigma\left(\frac{1}{2}\right)}} d s \\
& +\int_{0}^{t}\left\|(-A)^{\frac{1}{2}+\varepsilon} T(t-s)(T(h)-I)(-A)^{-\varepsilon} G(s)\right\|_{Y} d s \\
& \leq\|T(t+h) \varphi(0)-T(t) \varphi(0)\|_{Y}+\frac{2 C(R) D_{\frac{1}{2}}}{\frac{1}{2}-\sigma\left(\frac{1}{2}\right)} h^{\frac{1}{2}-\sigma\left(\frac{1}{2}\right)} \\
& +\int_{0}^{t}\left\|(-A)^{\frac{1}{2}+\varepsilon} T(t-s)\right\|_{\mathcal{L}(X, Y)}\left\|(T(h)-I)(-A)^{-\varepsilon} G(s)\right\| d s
\end{aligned}
$$




$$
\begin{gathered}
\leq\|T(t+h) \varphi(0)-T(t) \varphi(0)\|_{Y}+\frac{4 C(R) D_{\frac{1}{2}}}{1-2 \sigma\left(\frac{1}{2}\right)} h^{\frac{1}{2}-\sigma\left(\frac{1}{2}\right)} \\
\quad+\int_{0}^{t} \frac{D_{\frac{1}{2}+\varepsilon}}{(t-s)^{\frac{1}{2}+\varepsilon+\sigma\left(\frac{1}{2}+\varepsilon\right)}} \frac{C_{1-\varepsilon}}{\varepsilon} h^{\varepsilon}\|G(s)\| d s \\
\leq\|T(t+h) \varphi(0)-T(t) \varphi(0)\|_{Y}+\frac{4 C(R) D_{\frac{1}{2}}}{1-2 \sigma\left(\frac{1}{2}\right)} h^{\frac{1}{2}-\sigma\left(\frac{1}{2}\right)} \\
+\frac{4 C(R) D_{\frac{1}{2}+\varepsilon}}{1-2 \varepsilon-2 \sigma\left(\frac{1}{2}+\varepsilon\right)} \frac{C_{1-\varepsilon}}{\varepsilon} t^{\frac{1}{2}-\varepsilon-\sigma\left(\frac{1}{2}+\varepsilon\right)} h^{\varepsilon},
\end{gathered}
$$

onde $0<\varepsilon<r$. Da desigualdade acima obtemos que

$$
\begin{gathered}
\|\mathcal{T} u(t+h)-\mathcal{T} u(t)\|_{Y} \leq \| \\
T(t+h) \varphi(0)-T(t) \varphi(0) \|_{Y}+\frac{4 C(R) D_{\frac{1}{2}}}{1-2 \sigma\left(\frac{1}{2}\right)} h^{\frac{1}{2}-\sigma\left(\frac{1}{2}\right)} \\
+\frac{4 C(R) D_{\frac{1}{2}+\varepsilon}}{1-2 \varepsilon-2 \sigma\left(\frac{1}{2}+\varepsilon\right)} \frac{C_{1-\varepsilon}}{\varepsilon} b^{\frac{1}{2}-\varepsilon-\sigma\left(\frac{1}{2}+\varepsilon\right)} h^{\varepsilon},
\end{gathered}
$$

o que prova a continuidade à direita em $t \in[0, b]$, pois $T(t) \varphi(0) \in C([0, a], Y)$. De maneira similar, podemos mostrar a continuidade à esquerda para $t \in(0, b]$. Portanto $\mathcal{T} u \in C([0, b], Y)$.

Vejamos agora que $\mathcal{T F}_{R} \subset \mathcal{F}_{R}$. Note que para $u \in \mathcal{F}_{R}$ e $t \in[0, b]$,

$$
\begin{aligned}
\| \mathcal{T} u(t) & -\varphi(0) \|_{Y} \\
& \leq\|T(t) \varphi(0)-\varphi(0)\|_{Y}+\int_{0}^{t}\left\|(-A)^{\frac{1}{2}} T(t-s)\right\|_{\mathcal{L}(X, Y)}\|G(s)\| d s \\
& \leq\|T(t) \varphi(0)-\varphi(0)\|_{Y}+\int_{0}^{t} \frac{D_{\frac{1}{2}}}{(t-s)^{\frac{1}{2}+\sigma\left(\frac{1}{2}\right)}} 2 C(R) d s \\
& \leq\|T(t) \varphi(0)-\varphi(0)\|_{Y}+\frac{4 C(R) D_{\frac{1}{2}}}{1-2 \sigma\left(\frac{1}{2}\right)} b^{\frac{1}{2}-\sigma\left(\frac{1}{2}\right)},
\end{aligned}
$$

o que por (2.3) mostra que $\mathcal{T} u \in \mathcal{F}_{R}$. Como $u(\cdot)$ é arbitrário, segue-se que $\mathcal{T F}_{R} \subset \mathcal{F}_{R}$.

Para finalizar a demonstração, mostraremos que $\mathcal{T}$ é uma contração. Para $u, v \in \mathcal{F}_{R} \mathrm{e}$ $t \in[0, b]$, vemos que

$$
\begin{aligned}
\| \mathcal{T} u(t)- & \mathcal{T} v(t) \|_{Y} \\
\leq & \int_{0}^{t}\left\|(-A)^{\frac{1}{2}} T(t-s)\right\|_{\mathcal{L}(X, Y)}\left\|(-A)^{-\frac{1}{2}} g\left(s, u_{s}\right)-(-A)^{-\frac{1}{2}} g\left(s, v_{s}\right)\right\| d s \\
& \quad+\int_{0}^{t}\left\|(-A)^{\frac{1}{2}} T(t-s)\right\|_{\mathcal{L}(X, Y)}\left\|\widetilde{F}\left(s, u_{s}, u(s)\right)-\widetilde{F}\left(s, v_{s}, v(s)\right)\right\| d s \\
\leq & \left\|(-A)^{-\frac{1}{2}}\right\|_{\mathcal{L}(X)} L_{g}\left(R_{1}\right) K_{a, Y} \int_{0}^{t} \frac{D_{\frac{1}{2}}}{(t-s)^{\frac{1}{2}+\sigma\left(\frac{1}{2}\right)}} \sup _{\theta \in[0, s]}\|u(\theta)-v(\theta)\|_{Y} d s \\
& \quad+\left(L_{\widetilde{F}}^{1}\left(R_{1}\right) K_{a, Y}+L_{\widetilde{F}}^{2}\left(R_{1}\right)\right) \int_{0}^{t} \frac{D_{\frac{1}{2}}}{(t-s)^{\frac{1}{2}+\sigma\left(\frac{1}{2}\right)}} \sup _{\theta \in[0, s]}\|u(\theta)-v(\theta)\|_{Y} d s
\end{aligned}
$$




$$
\begin{aligned}
\leq \|( & -A)^{-\frac{1}{2}} \|_{\mathcal{L}(X)} L_{g}\left(R_{1}\right) K_{a, Y} \frac{2 D_{\frac{1}{2}}}{1-2 \sigma\left(\frac{1}{2}\right)} b^{\frac{1}{2}-\sigma\left(\frac{1}{2}\right)} d(u, v) \\
& +\left(L_{\widetilde{F}}^{1}\left(R_{1}\right) K_{a, Y}+L_{\widetilde{F}}^{2}\left(R_{1}\right)\right) \frac{2 D_{\frac{1}{2}}}{1-2 \sigma\left(\frac{1}{2}\right)} b^{\frac{1}{2}-\sigma\left(\frac{1}{2}\right)} d(u, v),
\end{aligned}
$$

o que segundo (2.4) mostra que existe $0<\Theta<1$ tal que $d(\mathcal{T} u, \mathcal{T} v) \leq \Theta d(u, v)$ para todo $u, v \in \mathcal{F}_{R}$. Isto completa a prova que $\mathcal{T}$ é uma contração em $\mathcal{F}_{R}$.

A existência de uma solução $Y$-fraca para o sistema (2.1)-(2.2) sobre [0, b], é agora uma conseqüência direta do princípio de ponto fixo para contrações. A prova está completa.

Nota 2.1.5. Nos próximos Lemas estudaremos diversas propriedades de regularidade da solução $Y$-fraca garantida pelo Teorema 2.1.4. Isto nos permitirá mostrar no Teorema 2.1.10 a existência de uma solução clássica para (2.1)-(2.2). No que segue, sempre assumiremos que as hipóteses do Teorema 2.1.4 são verificadas e que $u(\cdot)$ é a solução $Y$-fraca de (2.1)-(2.2) em $[0, b]$ para algum $0<b \leq a$. Além do anterior, $G:[0, b] \rightarrow Y$ será a função definida por $G(s)=(-A)^{-\frac{1}{2}} g\left(s, u_{s}\right)+\widetilde{F}\left(s, u_{s}, u(s)\right)$ e $R>0$ será tal que $\|G(s)\| \leq R$ para todo $s \in[0, b]$.

Lema 2.1.6. Se $\varphi(0) \in D\left((-A)^{\frac{1}{2}}\right)$ ent $\tilde{a} o \quad u \in C^{\min \left\{\frac{1}{2}-\sigma\left(\frac{1}{2}\right), \varepsilon\right\}}([0, b], Y)$ para todo $\varepsilon \in[0, r]$.

Prova. Se $t \in[0, b)$ e $h>0$ são tais que $t+h \leq b$, então

$$
\begin{aligned}
& \|u(t+h)-u(t)\|_{Y} \\
& \leq\|T(t+h) \varphi(0)-T(t) \varphi(0)\|_{Y} \\
& +\left\|\int_{0}^{t+h}(-A)^{\frac{1}{2}} T(t+h-s) G(s) d s-\int_{0}^{t}(-A)^{\frac{1}{2}} T(t-s) G(s) d s\right\|_{Y} \\
& \leq\left\|A \int_{0}^{h} T(t+s) \varphi(0) d s\right\|_{Y}+\int_{t}^{t+h}\left\|(-A)^{\frac{1}{2}} T(t+h-s) G(s)\right\|_{Y} d s \\
& +\int_{0}^{t}\left\|\left((-A)^{\frac{1}{2}} T(t+h-s)-(-A)^{\frac{1}{2}} T(t-s)\right) G(s)\right\|_{Y} d s \\
& \leq \int_{0}^{h}\left\|(-A)^{\frac{1}{2}} T(t+s)(-A)^{\frac{1}{2}} \varphi(0)\right\|_{Y} d s+R \int_{t}^{t+h} \frac{D_{\frac{1}{2}}}{(t+h-s)^{\frac{1}{2}+\sigma\left(\frac{1}{2}\right)}} d s \\
& +\int_{0}^{t}\left\|(-A)^{\frac{1}{2}+\varepsilon} T(t-s)(T(h)-I)(-A)^{-\varepsilon} G(s)\right\|_{Y} d s \\
& \leq\left\|(-A)^{\frac{1}{2}} \varphi(0)\right\| \int_{0}^{h}\left\|(-A)^{\frac{1}{2}} T(t+s)\right\|_{\mathcal{L}(X, Y)} d s+\frac{2 R D_{\frac{1}{2}}}{1-2 \sigma\left(\frac{1}{2}\right)} h^{\frac{1}{2}-\sigma\left(\frac{1}{2}\right)} \\
& +\int_{0}^{t}\left\|(-A)^{\frac{1}{2}+\varepsilon} T(t-s)\right\|_{\mathcal{L}(X, Y)} \int_{0}^{h}\left\|(-A)^{1-\varepsilon} T(\tau) G(s)\right\|_{Y} d \tau d s \\
& \leq\left\|(-A)^{\frac{1}{2}} \varphi(0)\right\| \int_{0}^{h} \frac{D_{\frac{1}{2}}}{(t+s)^{\frac{1}{2}+\sigma\left(\frac{1}{2}\right)}} d s+\frac{2 R D_{\frac{1}{2}}}{1-2 \sigma\left(\frac{1}{2}\right)} h^{\frac{1}{2}-\sigma\left(\frac{1}{2}\right)} \\
& +\int_{0}^{t} \frac{D_{\frac{1}{2}+\varepsilon}}{(t-s)^{\frac{1}{2}+\varepsilon+\sigma\left(\frac{1}{2}+\varepsilon\right)}} \int_{0}^{h} \frac{C_{1-\varepsilon}}{\tau^{1-\varepsilon}} R d \tau d s
\end{aligned}
$$




$$
\begin{aligned}
\leq & \frac{2 D_{\frac{1}{2}}\left\|(-A)^{\frac{1}{2}} \varphi(0)\right\|}{1-2 \sigma\left(\frac{1}{2}\right)} h^{\frac{1}{2}-\sigma\left(\frac{1}{2}\right)}+\frac{2 R D_{\frac{1}{2}}}{1-2 \sigma\left(\frac{1}{2}\right)} h^{\frac{1}{2}-\sigma\left(\frac{1}{2}\right)} \\
& +\frac{2 R b^{\frac{1}{2}-\varepsilon-\sigma\left(\frac{1}{2}+\varepsilon\right)} D_{\frac{1}{2}+\varepsilon} C_{1-\varepsilon}}{\left(1-2 \varepsilon-2 \sigma\left(\frac{1}{2}+\varepsilon\right)\right) \varepsilon} h^{\varepsilon},
\end{aligned}
$$

o qual implica que $u \in C^{\min \left\{\frac{1}{2}-\sigma\left(\frac{1}{2}\right), \varepsilon\right\}}([0, b], Y)$, pois $t$ e $h$ são arbitrários. A prova está completa.

No próximo Lema, $W(\cdot) \varphi$ é definida na seção 1.3 e $G(\cdot), R$ são como antes.

Lema 2.1.7. Assuma que as hipóteses do Lema 2.1.6 são satisfeitas e que existe $\gamma \in(0,1)$ tal que $W(\cdot) \varphi \in C^{\gamma}\left([0, b], \mathcal{B}_{Y}\right)$. Então a função $s \mapsto u_{s}$ pertence a

$$
C^{\min \left\{\frac{1}{2}-\sigma\left(\frac{1}{2}\right), \varepsilon, \gamma\right\}}\left([0, b], \mathcal{B}_{Y}\right) .
$$

Prova. Para mostrar o resultado introduzimos a decomposição $u(\cdot)=x(\cdot)+y(\cdot)$ onde $x, y$ : $(-\infty, b] \rightarrow Y$ são tais que $y_{0}=\varphi, x_{0}=0, y(t)=T(t) \varphi(0)$ para $t \in[0, b] \mathrm{e}$

$$
x(t)=\int_{0}^{t}(-A)^{\frac{1}{2}} T(t-\tau)\left((-A)^{\frac{1}{2}} g\left(\tau, u_{\tau}\right)+\widetilde{F}\left(\tau, u_{\tau}, u(\tau)\right)\right) d \tau, \quad t \in[0, b] .
$$

Da definição de $y(\cdot)$ é claro que $y_{t}=W(t) \varphi$ e que $u_{t}=y_{t}+x_{t}$ para cada $t \in[0, b]$.

Fazendo uso do Lema 2.1.6 e dos axiomas do espaço $\mathcal{B}_{Y}$, para $t \in[0, b)$ e $h>0$ tal que $t+h \leq b$ temos que

$$
\begin{aligned}
\left\|u_{t+h}-u_{t}\right\|_{\mathcal{B}_{Y}} & \leq K_{Y}(t) \sup _{0 \leq \theta \leq t}\|u(\theta+h)-u(\theta)\|_{Y}+M(t)\left\|u_{h}-\varphi\right\|_{\mathcal{B}_{Y}} \\
& =K_{a, Y} \sup _{0 \leq \theta \leq t}\|u(\theta+h)-u(\theta)\|_{Y}+M_{a, Y}\left\|x_{h}\right\|_{\mathcal{B}_{Y}}+M_{a, Y}\left\|y_{h}-\varphi\right\|_{\mathcal{B}_{Y}} \\
& \leq K_{a, Y} c_{1} h^{\min \left\{\frac{1}{2}-\sigma\left(\frac{1}{2}\right), \varepsilon\right\}}+M_{a, Y}\left\|x_{h}\right\|_{\mathcal{B}_{Y}}+M_{a, Y}\|W(h) \varphi-\varphi\|_{\mathcal{B}_{Y}} \\
& \leq c_{2} h^{\min \left\{\frac{1}{2}-\sigma\left(\frac{1}{2}\right), \varepsilon, \gamma\right\}}+M_{a, Y}\left\|x_{h}\right\|_{\mathcal{B}_{Y}} \\
& \leq c_{2} h^{\min \left\{\frac{1}{2}-\sigma\left(\frac{1}{2}\right), \varepsilon, \gamma\right\}}+M_{a, Y} K_{a, Y} \sup _{0 \leq \theta \leq h}\|x(\theta)\|_{Y} .
\end{aligned}
$$

Assim para completar a prova, é suficiente mostrar que existe $c>0$ (independente de $h \mathrm{e}$ $t \in[0, b])$ tal que $\sup _{0 \leq \theta \leq h}\|x(\theta)\|_{Y} \leq \operatorname{ch}^{\frac{1}{2}-\sigma\left(\frac{1}{2}\right)}$. Veja que para $\theta \in[0, h]$

$$
\begin{aligned}
\|x(\theta)\|_{Y} & \leq \int_{0}^{\theta}\left\|(-A)^{\frac{1}{2}} T(\theta-\tau)\right\|_{\mathcal{L}(X, Y)}\|G(\tau)\| d \tau \\
& \leq \int_{0}^{\theta} \frac{R D_{\frac{1}{2}}}{(\theta-\tau)^{\frac{1}{2}+\sigma\left(\frac{1}{2}\right)}} d \tau \\
& \leq \frac{R D_{\frac{1}{2}}}{\frac{1}{2}-\sigma\left(\frac{1}{2}\right)} \theta^{\frac{1}{2}-\sigma\left(\frac{1}{2}\right)} \\
& \leq \frac{2 R D_{\frac{1}{2}}}{1-2 \sigma\left(\frac{1}{2}\right)} h^{\frac{1}{2}-\sigma\left(\frac{1}{2}\right)},
\end{aligned}
$$




\section{Capítulo 2. Soluções em $L^{p}$ para sistemas do tipo Navier-Stokes com memória 16 não limitada}

o qual mostra que $\sup _{0 \leq \theta \leq h}\|x(\theta)\|_{Y} \leq \frac{2 R D_{\frac{1}{2}}}{1-2 \sigma\left(\frac{1}{2}\right)} h^{\frac{1}{2}-\sigma\left(\frac{1}{2}\right)}$. Isto completa a prova que $s \mapsto u_{s}$ é Hölder. Mais ainda, é fácil ver que $s \mapsto u_{s} \in C^{\min \left\{\frac{1}{2}-\sigma\left(\frac{1}{2}\right), \varepsilon, \gamma\right\}}\left([0, b], \mathcal{B}_{Y}\right)$. A prova está completa.

Lema 2.1.8. Suponha que as hipóteses do Lema 2.1.7 são verificadas. Então $u(t) \in$ $\left[D\left((-A)^{\frac{1}{2}}\right)\right]$ para cada $t \in[0, b]$.

Prova. Dos Lemas 2.1.6, 2.1.7 e das condições (iii) e (iv) segue que $G \in C^{\delta}([0, b] ; X)$ para algum $\delta \in(0,1)$. Reescrevamos agora $u(\cdot)$ na forma

$u(t)=T(t) \varphi(0)+\int_{0}^{t}(-A)^{\frac{1}{2}} T(t-s) G(t) d s+\int_{0}^{t}(-A)^{\frac{1}{2}} T(t-s)[G(s)-G(t)] d s, \quad t \in[0, b]$.

Como $(T(t))_{t \geq 0}$ é analítico e $\varphi(0) \in D\left((-A)^{\frac{1}{2}}\right)$, obtemos que $T(t) \varphi(0) \in D\left((-A)^{\frac{1}{2}}\right)$ e $(-A)^{\frac{1}{2}} T(t) \varphi(0)=T(t)(-A)^{\frac{1}{2}} \varphi(0)$ para todo $t \in[0, b]$. Mais ainda, do item (ii) do Teorema 1.1.5 sabemos que $\int_{0}^{t} T(t-s) G(t) d s \in D(A)$ e que

$$
A \int_{0}^{t} T(t-s) G(t) d s=T(t) G(t)-G(t)
$$

para todo $t \in[0, a]$. Usando agora que $(-A)^{\frac{1}{2}}$ é fechado, obtemos que

$$
(-A)^{\frac{1}{2}} \int_{0}^{t}(-A)^{\frac{1}{2}} T(t-s) G(t) d s=-A \int_{0}^{t} T(t-s) G(t) d s=G(t)-T(t) G(t),
$$

o que mostra que $\int_{0}^{t}(-A)^{\frac{1}{2}} T(t-s) G(t) d s \in D\left((-A)^{\frac{1}{2}}\right)$. Assim para completar a prova, é necessário mostrar que

$$
\int_{0}^{t}(-A)^{\frac{1}{2}} T(t-s)[G(s)-G(t)] d s \in D\left((-A)^{\frac{1}{2}}\right),
$$

para todo $t \in[0, b]$. Para mostrar isto, observamos que para $t \in[0, b]$ e $s \in[0, t]$

$$
\begin{aligned}
\left\|(-A)^{\frac{1}{2}}(-A)^{\frac{1}{2}} T(t-s)[G(t)-G(s)]\right\| & \leq\|A T(t-s)\|_{\mathcal{L}(X)}\|G(t)-G(s)\| \\
& \leq \frac{C_{1}}{(t-s)} c(t-s)^{\delta} \leq \frac{C_{1} c}{(t-s)^{1-\delta}}
\end{aligned}
$$

onde $c>0$ é independente de $t, s \in[0, a]$. Esta desigualdade e o fato que a função $s \mapsto$ $(-A)^{\frac{1}{2}}(-A)^{\frac{1}{2}} T(t-s)[G(t)-G(s)] \in C([0, t), X)$, mostra que $s \mapsto A T(t-s)[G(t)-G(s)]$ é uma função integrável sobre $[0, t)$ para todo $t \in[0, b]$. Mais ainda, como $(-A)^{\frac{1}{2}}$ é fechado segue que $\int_{0}^{t}(-A)^{\frac{1}{2}} T(t-s)[G(t)-G(s)] d s \in D\left((-A)^{\frac{1}{2}}\right)$ para cada $t \in[0, b)$. Isto completa a prova.

Proposição 2.1.9. Assuma que as hipóteses do Lema 2.1.8 são verificadas e que $\varphi(0) \in D(A)$. Então $u(\cdot)$ é uma solução fraca de $(2.1)-(2.2)$ em $[0, b]$ e u(.) pertence a $C^{\beta}\left([0, b],\left[D\left((-A)^{\frac{1}{2}}\right)\right]\right)$ para $\beta \in\left(0, \frac{1}{2}\right)$. 
Prova. Do Lema 2.1 .8 sabemos que $u(t) \in D\left((-A)^{\frac{1}{2}}\right)$ para todo $t \in[0, b]$. Assim, $F\left(s, u_{s}, u(s)\right)=(-A)^{\frac{1}{2}} \widetilde{F}\left(s, u_{s}, u(s)\right) \mathrm{e}$

$$
u(t)=T(t) \varphi(0)+\int_{0}^{t} T(t-s)\left[g\left(s, u_{s}\right)+F\left(s, u_{s}, u(s)\right)\right] d s,
$$

para todo $t \in[0, b]$. Isto mostra que $u(\cdot)$ é uma solução fraca de $(2.1)-(2.2)$ em $[0, b]$.

Agora mostraremos que $u(\cdot)$ é localmente Hölder contínua com respeito à norma em $\left[D\left((-A)^{\frac{1}{2}}\right)\right]$. Para $t \in[0, b)$ e $h>0$ tal que $t+h \in(0, b]$ vemos que

$$
\begin{aligned}
& \|u(t+h)-u(t)\|_{\frac{1}{2}} \\
& \leq\left\|(-A)^{\frac{1}{2}} T(t+h) \varphi(0)-(-A)^{\frac{1}{2}} T(t) \varphi(0)\right\|+\left\|(-A)^{\frac{1}{2}} \int_{t}^{t+h} T(t+h-s) G(s) d s\right\| \\
& +\left\|(-A)^{\frac{1}{2}} \int_{0}^{t}(T(h)-I) T(t-s) G(s) d s\right\| \\
& \leq\left\|A \int_{0}^{h}(-A)^{\frac{1}{2}} T(t+s) \varphi(0) d s\right\|+\int_{t}^{t+h}\left\|(-A)^{\frac{1}{2}} T(t+h-s)\right\|_{\mathcal{L}(X)}\|G(s)\| d s \\
& +\left\|\int_{0}^{t}(-A)^{\frac{1}{2}+\varepsilon} T(t-s)(T(h)-I)(-A)^{-\varepsilon} G(s) d s\right\| \\
& \leq \int_{0}^{h}\left\|(-A)^{\frac{1}{2}} T(t+s)\right\|_{\mathcal{L}(X)}\|A \varphi(0)\| d s+R \int_{t}^{t+h} \frac{C_{\frac{1}{2}}}{(t+h-s)^{\frac{1}{2}}} d s \\
& +\int_{0}^{t}\left\|(-A)^{\frac{1}{2}+\varepsilon} T(t-s)\right\|_{\mathcal{L}(X)} \int_{0}^{h}(-A)^{1-\varepsilon} T(\tau) G(s) \| d \tau d s \\
& \leq\|A \varphi(0)\| \int_{0}^{h} \frac{C_{\frac{1}{2}}}{(t+s)^{\frac{1}{2}}} d s+2 R C_{\frac{1}{2}} h^{\frac{1}{2}} \\
& +\int_{0}^{t} \frac{C_{\frac{1}{2}+\varepsilon}}{(t-s)^{\frac{1}{2}+\varepsilon}} \frac{C_{1-\varepsilon}}{\varepsilon} h^{\varepsilon} R d s \\
& \leq 2 C_{\frac{1}{2}}\|A \varphi(0)\| h^{\frac{1}{2}}+2 R C_{\frac{1}{2}} h^{\frac{1}{2}}+\frac{2 C_{1-\varepsilon} b^{\frac{1}{2}-\varepsilon} R C_{\frac{1}{2}+\varepsilon}}{\varepsilon(1-2 \varepsilon)} h^{\varepsilon},
\end{aligned}
$$

para $\varepsilon \in\left(0, \frac{1}{2}\right)$, o qual completa a demonstração pois $t$ e $h$ são arbitrários.

Como consequência dos resultados anteriores, no próximo Teorema estabelecemos condições sob as quais a $Y$-solução fraca $u(\cdot)$ de (2.1)-(2.2) é uma solução clássica de (2.1)-(2.2) em $[0, b]$.

Teorema 2.1.10. Seja $\varphi \in \mathcal{B}_{Y}$ e assuma que as condições $(i)-(i v)$ são verificadas. Se $\varphi(0) \in D(A)$ e existe $\gamma \in(0,1)$ tal que $W(\cdot) \varphi \in C^{\gamma}\left([0, b], \mathcal{B}_{Y}\right)$ então $u(\cdot)$ é uma solução clássica de (2.1)-(2.2) em [0,b].

Prova. Como conseqüência dos Lemas 2.1.6, 2.1.7, 2.1.8 e da Proposição 2.1.9, sabemos que $u \in C^{\beta}\left([0, b],\left[(-A)^{\frac{1}{2}}\right]\right)$ e que existe $\delta \in(0,1)$ tal que a função $s \mapsto u_{s}$ pertence a $C^{\min \left\{\frac{1}{2}-\sigma\left(\frac{1}{2}\right), \varepsilon, \delta\right\}}\left([0, b], \mathcal{B}_{Y}\right)$. Agora, das propriedades de $g(\cdot)$ e $F(\cdot)$, segue que 
$G(\cdot) \in C^{\min \left\{\frac{1}{2}-\sigma\left(\frac{1}{2}\right), \gamma, \gamma_{1}, \gamma_{2}\right\}}([0, b], X) . \quad$ O resultado segue então diretamente do Teorema 1.2.15.

\subsection{Existência de soluções para uma equação de Navier-Stokes com retardamento não limitado}

Nesta seção aplicamos os resultados da seção anterior no estudo da existência de soluções para uma classe de equações do tipo Navier Stokes com retardamento não limitado descritas na forma

$$
\begin{aligned}
u^{\prime}(t, x) & =\nu \Delta u(t, x)-\left(\left(\int_{-\infty}^{t} \alpha(s-t) u(s, x) d s+\gamma u(t-r, x)\right) \cdot \nabla\right) u(t, x) \\
& \quad+\nabla p(t, x)+\int_{-\infty}^{t} \beta(s-t) u(s, x) d s, \quad(t, x) \in[0, a] \times \Omega, \\
\operatorname{div} u(t) & =0, \quad t \in[0, a], \\
u(t, x) & =0, \quad(t, x) \in[0, a] \times \partial \Omega, \\
u_{0}(t, x) & =\varphi(t, x), \quad(t, x) \in(-\infty, 0] \times \Omega,
\end{aligned}
$$

sendo $\Omega \subset \mathbb{R}^{n}$ um aberto com fronteira $\partial \Omega$ de classe $C^{2}, \gamma \in \mathbb{R}$ e $\alpha(\cdot), \beta(\cdot), \varphi(\cdot)$ funções apropriadas, não negativas, que satisfazem

$$
\int_{-\infty}^{0} \frac{\alpha(s)^{q^{\prime}}}{\rho(s)^{\frac{q^{\prime}}{q}}} d s<\infty \quad \text { e } \quad \int_{-\infty}^{0} \frac{\beta(s)^{p^{\prime}}}{\rho(s)^{\frac{p^{\prime}}{p}}} d s<\infty,
$$

onde $q^{\prime}$ e $p^{\prime}$ são os expoentes conjugados de $q$ e $p$ respectivamente.

No que segue, introduzimos diferentes condições técnicas de maneira a poder aplicar os resultados abstratos da seção 2.1 no estudo do sistema hereditário (2.5)-(2.8). Para $n \in \mathbb{N}$ e $p>1$ introduzimos os espaços $E_{p}=\left(L^{p}(\Omega)\right)^{n} \mathrm{e}$

$$
\mathcal{V}=\left\{u \in\left(C_{0}^{\infty}(\Omega)\right)^{n} ; \operatorname{div} u=0\right\}
$$

Sejam agora $X_{p}=\overline{\mathcal{V}}^{E_{p}}, V_{p}=\overline{\mathcal{V}}^{\left(W_{0}^{1, p}(\Omega)\right)^{n}}$ e $P$ a projeção de $E_{p}$ sobre $X_{p}$. É conhecido da teoria clássica de equações de Navier-Stokes (veja, por exemplo, $[9,11]$ ) que

$$
E_{p}=X_{p} \oplus\left\{\nabla \phi ; \phi \in\left(W^{1, p}(\Omega)\right)^{n}\right\}
$$

e que $A_{p}=\nu P \Delta$ é o gerador infinitesimal de um semigrupo analítico $(T(t))_{t \geq 0}$ em $X_{p}$. Se aplicarmos a projeção $P$ sobre o sistema (2.5)-(2.8) obtemos

$$
\begin{aligned}
\frac{d}{d t} u(t) & =\nu P \Delta u(t)+F\left(t, u_{t}, u(t)\right)+g\left(t, u_{t}\right), \quad t \geq 0, \\
u_{0} & =P \varphi
\end{aligned}
$$


onde temos introduzido a notação

$$
\begin{aligned}
F\left(t, u_{t}, u(t)\right) & =-P\left(\left(\int_{-\infty}^{t} \alpha(s-t) u(s) d s+\gamma u(t-r)\right) \cdot \nabla\right) u(t) \\
& =-P\left(\left(\int_{-\infty}^{0} \alpha(s) u_{t}(s) d s+\gamma u_{t}(-r)\right) \cdot \nabla\right) u(t),
\end{aligned}
$$

e

$$
g\left(t, u_{t}\right)=P \int_{-\infty}^{t} \beta(s-t) u(s) d s=P \int_{-\infty}^{0} \beta(s) u_{t}(s) d s
$$

Para mostrar a existência de soluções para (2.5)-(2.8) precisamos do seguinte Lema o qual resume várias propriedades de teoria de semigrupos associadas a os operadores $A_{p}$.

Lema 2.2.1. As seguintes propriedades são verificadas.

1. $A_{p}=\nu P \Delta$ é o gerador infinitesimal de um semigrupo analítico $\left(T_{p}(t)\right)_{t \geq 0}$ em $X_{p}$.

2. Para $1<p<q<\infty, T_{q}(t)=\left.T_{p}(t)\right|_{X_{q}} e(-A)_{p}^{\frac{1}{2}} T_{p}(t) \in \mathcal{L}\left(X_{p}, X_{q}\right)$ para todo $t>0$. Mais ainda, existe $C_{p, q}>0$ tal que $\left\|(-A)^{\alpha} T_{p}(t)\right\|_{\mathcal{L}\left(X_{p}, X_{q}\right)} \leq \frac{C_{p, q}}{t^{\alpha+n\left(\frac{1}{p}-\frac{1}{q}\right)}}$ para todo $t>0$ $e \alpha \geq 0$.

3. Para cada $0<k \leq n$, existe $B_{k} \in \mathcal{L}\left(E_{p}, X_{p}\right)$ tal que $P \frac{\partial}{\partial x_{k}}=(-A)_{p}^{\frac{1}{2}} B_{k}$.

Prova. As propriedades seguem diretamente de [11], e [1] respectivamente.

Note que, se no item 2 do teorema anterior $q=2 p$ e $p>n$ então $\sigma=\sigma\left(X_{p}, X_{q}\right)=$ $n\left(\frac{1}{p}-\frac{1}{q}\right)<\frac{1}{2}$. Assim, podemos escolher $0<r<\frac{1}{2}-\sigma$, de forma que $\alpha \leq \frac{1}{2}+r<1-\sigma$ e a condição $\sigma<1-\alpha$ da hipótese (ii) fica cumprida. Deste ponto em diante consideramos então sempre $q=2 p$.

É agora necessário introduzir espaços de fase (espaços das histórias) apropriados, $\mathcal{B}_{q}$, de modo de transformar o sistema (2.5)-(2.8) na forma abstrata (2.1)-(2.2). Notamos que a escolha de $\mathcal{B}_{q}$ tem relação direta com as propriedades que desejamos para as funções $F(\cdot)$, $g(\cdot)$. Neste caso, por agora apenas formalmente, estas funções são

$$
\begin{aligned}
F(t, \psi, w) & =-P\left(\left(\int_{-\infty}^{0} \alpha(s) \psi(s) d s+\gamma \psi(-r)\right) \cdot \nabla\right) w \\
g(t, \psi) & =P \int_{-\infty}^{0} \beta(s) \psi(s) d s,
\end{aligned}
$$

para $\psi=\left(\psi_{1}, \ldots, \psi_{n}\right) \in \mathcal{B}_{X_{p}}, w=\left(w_{1}, \ldots, w_{n}\right) \in X_{p}$. Considerando os termos integrais que aparecem na definição das funções anteriores, parece razoável escolher espaços do tipo $\mathcal{B}_{X_{q}}=\left(C_{r} \times L^{q}\left(\rho ; X_{q}\right)\right)^{n}, q>1, r>0$, como espaços de fase para modelar o problema. No que segue, $F_{j}$ representa a $j$-ésima coordenada do vetor $F \in \mathbb{R}^{n}$. Assumindo que $\psi \in \mathcal{B}_{X_{q}}$ 
e $w \in X_{q}$ são suficientemente regulares de modo que $\operatorname{div}\left(\psi_{1}, \ldots, \psi_{n}\right)=0$ e $\operatorname{div} w=0$, segue que

$$
\begin{aligned}
F_{j}(t, \psi, w) & =-\sum_{i=1}^{n} \int_{-\infty}^{0} \alpha(s) \psi_{i}(s) d s \frac{\partial}{\partial x_{i}} w_{j}+\sum_{i=1}^{n} \gamma \psi_{i}(-r) \frac{\partial}{\partial x_{i}} w_{j} \\
& =-\sum_{i=1}^{n} \frac{\partial}{\partial x_{i}}\left(\int_{-\infty}^{0} \alpha(s) \psi_{i}(s) d s w_{j}\right)+\sum_{i=1}^{n} \frac{\partial}{\partial x_{i}}\left(\gamma \psi_{i}(-r) w_{j}\right) \\
& =-\sum_{i=1}^{n} \frac{\partial}{\partial x_{i}}\left(\int_{-\infty}^{0} \alpha(s) \psi_{i}(s) d s w_{j}+\gamma \psi_{i}(-r) w_{j}\right) .
\end{aligned}
$$

De acordo com [1], existem operadores limitados $B_{k}: E_{p} \rightarrow X_{p}$ tais que $(-A)^{\frac{1}{2}} B_{k}=P \frac{\partial}{\partial x_{k}}$. Mais ainda, estes operadores aplicam $X_{2 p}$ em $X_{p}$. O que nos leva a

$$
F(t, \psi, w)=(-A)^{\frac{1}{2}}\left(-\sum_{i=1}^{n} B_{i}\left(\int_{-\infty}^{0} \alpha(s) \psi_{i}(s) d s w_{j}+\gamma \psi_{i}(-r) w_{j}\right)\right)_{j}
$$

Definamos agora, $\widetilde{F}:[0, a] \times \mathcal{B}_{X_{q}} \times X_{q} \rightarrow X_{p}$ por $\widetilde{F}(t, \psi, w)=\left(\widetilde{F}_{1}(t, \psi, w), \ldots, \widetilde{F}_{n}(t, \psi, w)\right)$, onde

$$
\widetilde{F}_{j}(t, \psi, w)=-\sum_{i=1}^{n} B_{i}\left(\int_{-\infty}^{0} \alpha(s) \psi_{i}(s) d s w_{j}\right)-\sum_{i=1}^{n}\left(B_{i} \gamma \psi_{i}(-r) w_{j}\right) .
$$

Mostraremos a seguir que $\widetilde{F}$ satisfaz a condição de Lipschitz na hipótese (iii). Seja $b=$ $\max _{1 \leq i \leq n}\left\{\left\|B_{i}\right\|_{\mathcal{L}\left(E_{p}, X_{p}\right)}\right\}$, e assuma que $\rho(\cdot)$ é uma função que verifica as condições $\left(g_{1}\right)-\left(g_{5}\right)$ na nomenclatura de [14]. Dados então $(t, \psi, w),(t, \varphi, v) \in[0, a] \times \mathcal{B}_{X_{q}} \times X_{q}$, temos que

$$
\begin{aligned}
& \| \widetilde{F}_{j}(t, \psi, w)- \widetilde{F}_{j}(t, \varphi, v) \|_{p} \\
&=\left\|\widetilde{F}_{j}(t, \psi, w)-\widetilde{F}_{j}(t, \psi, v)+\widetilde{F}_{j}(t, \psi, v)-\widetilde{F}_{j}(t, \varphi, v)\right\|_{p} \\
&=\| \sum_{i=1}^{n}\left[B_{i}\left(\int_{-\infty}^{0} \alpha(s) \psi_{i}(s) d s\left(w_{j}-v_{j}\right)\right)+B_{i} \gamma \psi_{i}(-r)\left(w_{j}-v_{j}\right)\right] \\
& \quad+\sum_{i=1}^{n}\left[B_{i}\left(\int_{-\infty}^{0} \alpha(s)\left(\psi_{i}(s)-\varphi_{i}(s)\right) d s v_{j}\right)+B_{i} \gamma\left(\left(\psi_{i}(-r)-\varphi_{i}(-r)\right) v_{j}\right)\right] \|_{p} \\
& \leq b \sum_{i=1}^{n}\left\|\int_{-\infty}^{0} \alpha(s) \psi_{i}(s) d s\left(w_{j}-v_{j}\right)+\int_{-\infty}^{0} \alpha(s)\left(\psi_{i}(s)-\varphi_{i}(s)\right) d s v_{j}\right\|_{p} \\
& \quad+b \gamma \sum_{i=1}^{n}\left\|\left(\psi_{i}(-r)-\varphi_{i}(-r)\right) v_{j}+\psi_{i}(-r)\left(w_{j}-v_{j}\right)\right\|_{p} .
\end{aligned}
$$

A norma na primeira parcela desta soma satisfaz,

$$
\begin{aligned}
& \left\|\int_{-\infty}^{0} \alpha(s) \psi_{i}(s) d s\left(w_{j}-v_{j}\right)+\int_{-\infty}^{0} \alpha(s)\left(\psi_{i}(s)-\varphi_{i}(s)\right) d s v_{j}\right\|_{p} \\
& \leq\left\|\int_{-\infty}^{0} \alpha(s) \psi_{i}(s) d s\left(w_{j}-v_{j}\right)\right\|_{p}+\left\|\int_{-\infty}^{0} \alpha(s)\left(\psi_{i}(s)-\varphi_{i}(s)\right) d s v_{j}\right\|_{p}
\end{aligned}
$$




$$
\leq\left\|\int_{-\infty}^{0} \alpha(s) \psi_{i}(s) d s\right\|_{2 p}\left\|w_{j}-v_{j}\right\|_{2 p}+\left\|\int_{-\infty}^{0} \alpha(s)\left(\psi_{i}(s)-\varphi_{i}(s)\right) d s\right\|_{2 p}\left\|v_{j}\right\|_{2 p} .
$$

Sejam $q^{\prime}$ o expoente conjugado de $q=2 p$ e $C_{\rho}=\int_{-r}^{0} \rho(s) d s$ e assuma que

$$
C_{\alpha, \rho}=\left(\int_{-\infty}^{0} \frac{\alpha(s)^{q^{\prime}}}{\rho(s)^{\frac{q^{\prime}}{q}}} d s\right)^{\frac{1}{q^{\prime}}}<\infty
$$

Então vemos que

$$
\begin{aligned}
& \left\|\int_{-\infty}^{0} \alpha(s) \psi_{i}(s) d s\right\|_{q}^{q}=\left\|\int_{-\infty}^{0} \frac{\alpha(s)}{\rho(s)^{\frac{1}{q}}} \rho(s)^{\frac{1}{q}} \psi_{i}(s) d s\right\|_{q}^{q} \\
& =\left\|\left(\int_{-\infty}^{0} \frac{\alpha(s)^{q^{\prime}}}{\rho(s)^{\frac{q^{\prime}}{q}}} d s\right)^{\frac{1}{q^{\prime}}}\left(\int_{-\infty}^{0} \rho(s)\left(\psi_{i}(s)\right)^{q} d s\right)^{\frac{1}{q}}\right\|_{q}^{q} \\
& =\left(\int_{-\infty}^{0} \frac{\alpha(s)^{q^{\prime}}}{\rho(s)^{\frac{q^{\prime}}{q}}} d s\right)^{\frac{q}{q^{\prime}}}\left\|\left(\int_{-\infty}^{0} \rho(s)\left(\psi_{i}(s)\right)^{q} d s\right)^{\frac{1}{q}}\right\|_{q}^{q} \\
& \leq C_{\alpha, \rho}^{q} \int_{\Omega} \int_{-\infty}^{0} \rho(s)\left|\psi_{i}(s)\right|^{q} d s d x \\
& =C_{\alpha, \rho}^{q} \int_{-\infty}^{0} \rho(s) \int_{\Omega}\left|\psi_{i}(s)\right|^{q} d x d s \\
& =C_{\alpha, \rho}^{q} \int_{-\infty}^{0} \rho(s)\left\|\psi_{i}(s)\right\|_{q}^{q} d s \\
& =C_{\alpha, \rho}^{q}\left(\int_{-\infty}^{-r} \rho(s)\left\|\psi_{i}(s)\right\|_{q}^{q} d s+\int_{-r}^{0} \rho(s)\left\|\psi_{i}(s)\right\|_{q}^{q} d s\right) \\
& \leq C_{\alpha, \rho}^{q}\left(\sup _{-r \leq s \leq 0}\left\|\psi_{i}(s)\right\|_{q}^{q} \int_{-r}^{0} \rho(s) d s+\int_{-\infty}^{-r} \alpha(s)\left\|\psi_{i}(s)\right\|_{q}^{q} d s\right) \\
& \leq C_{\alpha, \rho}^{q}\left(C_{\rho} \sup _{-r \leq s \leq 0}\left\|\psi_{i}(s)\right\|_{q}^{q}+\int_{-\infty}^{-r} \rho(s)\left\|\psi_{i}(s)\right\|_{q}^{q} d s\right) \\
& \leq C_{\alpha, \rho}^{q} \max \left\{C_{\rho}, 1\right\}\left(\sup _{-r \leq s \leq 0}\left\|\psi_{i}(s)\right\|_{q}^{q}+\left(\int_{-\infty}^{-r} \rho(s)\left\|\psi_{i}(s)\right\|_{q}^{q} d s\right)^{\frac{q}{q}}\right) \\
& \leq C_{\alpha, \rho}^{q} \max \left\{C_{\rho}, 1\right\}\left(\sup _{-r \leq s \leq 0}\left\|\psi_{i}(s)\right\|_{q}+\left(\int_{-\infty}^{-r} \rho(s)\left\|\psi_{i}(s)\right\|_{q}^{q} d s\right)^{\frac{1}{q}}\right)^{q} \\
& =C_{\alpha, \rho}^{q} \max \left\{C_{\rho}, 1\right\}\left\|\psi_{i}\right\|_{C_{r} \times L^{q}\left(\rho ; X_{q}\right)}^{q},
\end{aligned}
$$

Procedendo de maneira análoga podemos mostrar que

$$
\left\|\int_{-\infty}^{0} \alpha(s)\left(\psi_{i}(s)-\varphi_{i}(s)\right) d s\right\|_{q} \leq C_{\alpha, \rho} C\left\|\psi_{i}-\varphi_{i}\right\|_{C_{r} \times L^{q}\left(\rho ; X_{q}\right)},
$$

onde $C=\max \left\{C_{\rho}, 1\right\}$. Das estimativas anteriores segue que

$$
\left\|\int_{-\infty}^{0} \alpha(s) \psi_{i}(s) d s\left(w_{j}-v_{j}\right)+\int_{-\infty}^{0} \alpha(s)\left(\psi_{i}(s)-\varphi_{i}(s)\right) d s v_{j}\right\|_{p}
$$




$$
\leq C_{\alpha, \rho} C\left\|\psi_{i}\right\|_{C_{r} \times L^{q}\left(\rho ; X_{q}\right)}\left\|w_{j}-v_{j}\right\|_{q}+C_{\alpha, \rho} C\left\|\psi_{i}-\varphi_{i}\right\|_{C_{r} \times L^{q}\left(\rho ; X_{q}\right)}\left\|v_{j}\right\|_{q}
$$

Por outro lado, veja agora que

$$
\begin{aligned}
\|\left(\psi_{i}(-r)-\right. & \left.\varphi_{i}(-r)\right) v_{j}+\psi_{i}(-r)\left(w_{j}-v_{j}\right) \|_{p} \\
& \leq\left\|\left(\psi_{i}(-r)-\varphi_{i}(-r)\right) v_{j}\right\|_{p}+\left\|\psi_{i}(-r)\left(w_{j}-v_{j}\right)\right\|_{p} \\
& \leq\left\|\psi_{i}(-r)-\varphi_{i}(-r)\right\|_{2 p}\left\|v_{j}\right\|_{2 p}+\left\|\psi_{i}(-r)\right\|_{2 p}\left\|w_{j}-v_{j}\right\|_{2 p} \\
& \leq\left\|\psi_{i}-\varphi_{i}\right\|_{C_{r} \times L^{q}\left(\rho ; X_{q}\right)}\left\|v_{j}\right\|_{2 p}+\left\|\psi_{i}\right\|_{C_{r} \times L^{q}\left(\rho ; X_{q}\right)}\left\|w_{j}-v_{j}\right\|_{2 p} .
\end{aligned}
$$

Destas estimativas anteriores obtemos finalmente que

$$
\begin{aligned}
& \left\|\widetilde{F}_{j}(t, \psi, w)-\widetilde{F}_{j}(t, \varphi, v)\right\|_{p} \\
& \leq b \sum_{i=1}^{n}\left(C_{\alpha, \rho} C+\gamma\right)\left\|\psi_{i}\right\|_{C_{r} \times L^{q}\left(\rho ; X_{q}\right)}\|w-v\|_{q} \\
& \quad+b \sum_{i=1}^{n}\left(C_{\alpha, \rho} C+\gamma\right)\left\|\psi_{i}-\varphi_{i}\right\|_{C_{r} \times L^{q}\left(\rho ; X_{q}\right)}\|v\|_{q} \\
& \leq b n\left(C_{\alpha, \rho} C+\gamma\right)\|\psi\|_{\mathcal{B}_{X_{q}}}\|w-v\|_{q}+b n\left(C_{\alpha, \rho} C+\gamma\right)\|\psi-\varphi\|_{\mathcal{B}_{X_{q}}}\|v\|_{q},
\end{aligned}
$$

para $C=\max \left\{C_{\rho}, 1\right\}$, o que mostra que $\widetilde{F}$ satisfaz a condição de Lipschitz da hipótese (iii), $\operatorname{com} L_{\widetilde{F}}^{0}(R)=0$ e

$$
L_{\widetilde{F}}^{1}(R)=L_{\widetilde{F}}^{2}(R)=\operatorname{bn}\left(C_{\alpha, \rho}^{q} C+\gamma\right) R .
$$

Estimativas similares às anteriores permitem mostrar que $g(s, \cdot)$ é um operador linear limitado para cada $s \in[0, a]$ e que $\|g(s, \cdot)\|_{\mathcal{L}\left(\mathcal{B}_{X_{p}}, X_{p}\right)} \leq L_{g}$. De fato, seja $p^{\prime}$ é o expoente conjugado de $p$ e

$$
C_{\beta, \rho}=\left(\int_{-\infty}^{0} \frac{\beta(s)^{p^{\prime}}}{\rho(s)^{\frac{p^{\prime}}{p}}} d s\right)^{\frac{1}{p^{\prime}}}<\infty .
$$

Para $\psi_{1}, \psi_{2} \in \mathcal{B}_{X_{p}}$, vemos que

$$
\begin{aligned}
\| g\left(t, \psi_{1}\right) & -g\left(t, \psi_{2}\right) \|_{p}^{p} \\
& \leq\left\|\int_{-\infty}^{0} \beta(s)\left(\psi_{1}(s)-\psi_{2}(s)\right) d s\right\|_{p}^{p} \\
& \leq\left\|\int_{-\infty}^{0} \frac{\beta(s)}{\rho(s)^{\frac{1}{p}}} \rho(s)^{\frac{1}{p}}\left(\psi_{1}(s)-\psi_{2}(s)\right) d s\right\|_{p}^{p} \\
& \leq\left\|\left(\int_{-\infty}^{0} \frac{\beta(s)^{p^{\prime}}}{\rho(s)^{\frac{p^{\prime}}{p}}} d s\right)^{\frac{1}{p^{\prime}}}\left(\int_{-\infty}^{0} \rho(s)\left(\psi_{1}(s)-\psi_{2}(s)\right)^{p} d s\right)^{\frac{1}{p}}\right\|_{p}^{p} \\
& \leq C_{\beta, \rho}^{p} \int_{\Omega} \int_{-\infty}^{0} \rho(s)\left|\psi_{1}(s)-\psi_{2}(s)\right|^{p} d s d x
\end{aligned}
$$




$$
\begin{aligned}
& =C_{\beta, \rho}^{p} \int_{-\infty}^{0} \rho(s)\left\|\psi_{1}(s)-\psi_{2}(s)\right\|_{p}^{p} d s \\
& =C_{\beta, \rho}^{p}\left(\int_{-\infty}^{-r} \rho(s)\left\|\psi_{1}(s)-\psi_{2}(s)\right\|_{p}^{p} d s+\int_{-r}^{0} \rho(s)\left\|\psi_{1}(s)-\psi_{2}(s)\right\|_{p}^{p} d s\right) \\
& \leq C_{\beta, \rho}^{p}\left(\sup _{-r \leq s \leq 0}\left\|\psi_{1}(s)-\psi_{2}(s)\right\|_{p}^{p} \int_{-r}^{0} \rho(s) d s+\int_{-\infty}^{-r} \rho(s)\left\|\psi_{1}(s)-\psi_{2}(s)\right\|_{p}^{p} d s\right) \\
& \leq C_{\beta, \rho}^{p} \max \left\{C_{\rho}, 1\right\}\left(\sup _{-r \leq s \leq 0}\left\|\psi_{1}(s)-\psi_{2}(s)\right\|^{p}+\left(\int_{-\infty}^{-r} \rho(s)\left\|\psi_{1}(s)-\psi_{2}(s)\right\|_{p}^{p} d s\right)^{\frac{1}{p}}\right)^{p} \\
& \leq C_{\beta, \rho}^{p} \max \left\{C_{\rho}, 1\right\}\left\|\psi_{1}-\psi_{2}\right\|_{\mathcal{B}_{X_{p}}}^{p},
\end{aligned}
$$

e assim,

$$
\left\|g\left(t, \psi_{1}\right)-g\left(t, \psi_{2}\right)\right\|_{p} \leq C_{\beta, \rho} C\left\|\psi_{1}-\psi_{2}\right\|_{\mathcal{B}_{X_{p}}},
$$

onde $C=\max \left\{C_{\rho}, 1\right\}$.

O desenvolvimento anterior nos permite modelar o sistema (2.5)-(2.8) na forma abstrata

$$
\begin{aligned}
u^{\prime}(t) & =A u(t)+F\left(t, u_{t}, u(t)\right)+g\left(t, u_{t}\right), \quad t \in[0, a], \\
u_{0} & =\varphi \in \mathcal{B}
\end{aligned}
$$

em que as condições $(i)$-(iv) são verificadas. A aplicação dos resultados abstratos da primeira seção nos permitem mostrar o seguinte resultado. Na seguinte proposição, $(W(t))_{t \geq 0}$ é o semigrupo introduzido na seção 1.3 , com $\left(T_{p}(t)\right)_{t \geq 0}$ no lugar de $(T(t))_{t \geq 0}$.

Proposição 2.2.2. Seja $\varphi \in \mathcal{B}_{X_{2 p}}$ com $p>n$, e suponha que as condições anteriores são válidas. Então as seguintes propriedades são verificadas.

(a) Existe $b \in(0, a]$ e uma única solução $X_{2 p}$-fraca $u \in C\left([0, b], X_{2 p}\right)$ de (2.9)-(2.10) em $[0, b]$.

(b) Se $\varphi(0) \in H_{0}^{1}(\Omega)$ então $u \in C^{\min \left\{\frac{1}{2}-\sigma\left(\frac{1}{2}\right), r\right\}}\left([0, b], X_{2 p}\right)$.

(c) $S e \varphi(0) \in H_{0}^{1}(\Omega)$ e existe $\gamma \in(0,1)$ tal que $W(\cdot) \varphi \in C^{\gamma}\left([0, b], \mathcal{B}_{X_{2 p}}\right)$, então a função $s \mapsto u_{s} \in C^{\min \left\{\frac{1}{2}-\sigma\left(\frac{1}{2}\right), r, \gamma\right\}}\left([0, b], X_{2 p}\right)$ e $u(t) \in H_{0}^{1}(\Omega)$ para cada $t \in[0, b]$.

(d) Se $\varphi(0) \in H_{0}^{1}(\Omega) \cap W^{2}(\Omega)$ e existe $\gamma \in(0,1)$ tal que $W(\cdot) \varphi \in C^{\gamma}\left([0, b], \mathcal{B}_{X_{2 p}}\right)$, então $u(\cdot)$ é uma solução clássica de (2.9)-(2.10) em $[0, b], u \in C^{1}\left([0, b], X_{p}\right) \cap C\left([0, b], H_{0}^{1}(\Omega) \cap\right.$ $\left.W^{2}(\Omega)\right), u \in C^{\beta}\left([0, b], H_{0}^{1}(\Omega)\right)$ para $\beta \in\left(0, \frac{1}{2}\right)$ e $u(\cdot)$ satisfaz $(2.5)-(2.8)$ para quase todo $(t, \xi) \in(-\infty, b] \times \Omega$.

Uma vez que o vetor velocidade $u$ é conhecido, podemos agora determinar a pressão $p(t, x)$, usando os métodos descritos em [26]. A pressão será unicamente determinada, a menos de uma constante, pela própria equação (2.5). 
Capítulo 2. Soluções em $L^{p}$ para sistemas do tipo Navier-Stokes com memória 24 não limitada 


\title{
Capítulo 3
}

\section{Problema de Navier-Stokes com retardo para dimensão 3}

\begin{abstract}
Resumo do capítulo: Neste capítulo estudaremos a existência de soluções, em dimensão 3, para a equação do tipo Navier-Stokes

$$
\frac{\partial u}{\partial t}-\nu \Delta u+\left(F\left(t, u_{t}\right) \cdot \nabla\right) u+\nabla p=g\left(t, u_{t}\right)
$$

sujeita a condições iniciais, de retardo, de contorno e de divergência livre. Após a reformulação adequada do problema, enunciaremos resultados a respeito de existência de solução fraca (Teorema 3.2.1) e forte (Teorema 3.2.2). Como complemento mostramos que a solução forte define um conjunto absorvente (Corolário 3.2.5). Obtemos uma estimativa de energia para a solução fraca, e mostraremos assim que qualquer solução fraca que satisfaz tal estimativa de energia, converge para a solução estacionária quando $t \rightarrow \infty$ (Teorema 3.4.2).
\end{abstract}

\subsection{Formulação do problema e hipóteses}

Seja $\Omega$ um domínio aberto e limitado de $\mathbb{R}^{3}$ com fronteira suave e o seguinte sistema de equações de Navier-Stokes

$$
\begin{aligned}
\frac{\partial}{\partial t} u-\nu \Delta u+(u(t-\tau(t)) \cdot \nabla) u & =f-\nabla p+g\left(t, u_{t}\right) \quad \text { em } \quad(0,+\infty) \times \Omega, \\
\operatorname{div} u & =0 \quad \text { em } \quad(0,+\infty) \times \Omega \\
u & =0 \quad \text { sobre } \quad(0,+\infty) \times \partial \Omega \\
u(0, x) & =u^{0}(x) \quad \text { em } \quad \Omega \\
u(t, x) & =\phi(t, x) \quad \text { em } \quad(-h, 0) \times \Omega,
\end{aligned}
$$

onde $\nu>0$ é a viscosidade, $u$ é o campo velocidade, $p$ é a pressão hidrostática associada, $f$ é uma força externa sem retardo, $g$ é outra força externa com alguma característica hereditária (retardo), $u^{0}$ é a velocidade inicial, $\phi$ é o dado inicial no intervalo de tempo $(-h, 0)$, onde $h$ é um número positivo fixado, e $\tau$ é um retardo que assumiremos depender do tempo $t$. 
Para cada tempo $t \in(0, T)$, denotaremos por $u_{t}$ a função definida em $(-h, 0)$ pela relação $u_{t}(s)=u(t+s), s \in(-h, 0)$.

Queremos estudar a existência e o comportamento exponencial de soluções deste modelo. Para isto consideraremos os seguintes espaços usuais:

$$
\begin{aligned}
\mathcal{V} & =\left\{u \in\left(C_{0}^{\infty}(\Omega)\right)^{3} ; \operatorname{div} u=0\right\}, \\
H & =\text { o fecho de } \mathcal{V} \text { em }\left(L^{2}(\Omega)\right)^{3}, \\
V & =\text { o fecho de } \mathcal{V} \text { em }\left(H_{0}^{1}(\Omega)\right)^{3},
\end{aligned}
$$

e definimos a forma trilinear

$$
b(u, v, w)=\int_{\Omega}(u \cdot \nabla) v \cdot w d x=\sum_{i, j=1}^{3} \int_{\Omega} u_{i} \frac{\partial v_{j}}{\partial x_{i}} w_{j} d x
$$

para $u, v, w \in V$. A respeito desta forma trilinear, sabemos que para $n=3$, valem as estimativas,

$$
\begin{array}{rlrl}
|b(u, v, w)| & \leq C_{3}\|u\|_{L^{2}}^{\frac{1}{4}}\|u\|_{V}^{\frac{3}{4}}\|v\|_{V}\|w\|_{L^{2}}^{\frac{1}{4}}\|w\|_{V}^{\frac{3}{4}} \quad \forall \quad u, v, w \in V \\
|b(u, v, w)| & \leq C_{3}\|u\|_{V}\|v\|_{V}^{\frac{1}{2}}\|A v\|_{L^{2}}^{\frac{1}{2}}\|w\|_{L^{2}} \quad \forall & u \in V, v \in D(A), w \in H \\
b(u, v, v) & =0, \quad \forall v \in V .
\end{array}
$$

Para maiores detalhes a respeito destas estimativas, pode-se consultar Robinson [24, Proposição 9.2].

Sobre a força externa, que depende do retardo, $g:[0, T] \times C([-h, 0] ; V) \rightarrow\left(L^{2}(\Omega)\right)^{3}$, vamos assumir que satisfaz as mesmas hipóteses de Caraballo [2],

(H1) para todo $\xi \in C([-h, 0] ; V)$, as aplicações $t \in[0, T] \mapsto g(t, \xi) \in\left(L^{2}(\Omega)\right)^{3}$ são integráveis,

(H2) $g(t, 0)=0$ para cada $t \in[0, T]$,

(H3) existe $L_{g}>0$ tal que, para $t \in[0, T]$

$$
\|g(t, \xi)-g(t, \eta)\|_{L^{2}} \leq L_{g}\|\xi-\eta\|_{C([-h, 0] ; V)},
$$

(H4) existe $C_{g}>0$ tal que, para $t \in[0, T]$

$$
\int_{0}^{t}\left\|g\left(s, u_{s}\right)-g\left(s, v_{s}\right)\right\|_{L^{2}}^{2} d s \leq C_{g} \int_{-h}^{t}\|u(s)-v(s)\|_{V}^{2} d s
$$

(H5) se uma sequência $v_{k}$ converge fracamente para $v$ em $L^{2}(-h, T ; V)$ e fortemente em $L^{2}(-h, T ; H)$, então a sequência de funções $\xi_{k}$ dadas por $\xi_{k}(t)=g\left(t,\left(v_{k}\right)_{t}\right)$ converge fracamente para $\xi(t)=g\left(t, v_{t}\right)$ em $L^{2}\left(0, T ; V^{\prime}\right)$. 
Note que de (H1)-(H4) a aplicação

$$
G: u \in C([-h, T] ; V) \mapsto g\left(t, u_{t}\right) \in L^{2}\left(0, T ;\left(L^{2}(\Omega)\right)^{3}\right),
$$

para todo $t \in[0, T]$, admite uma única extensão a uma aplicação

$$
\widetilde{G}: L^{2}(-h, T ; V) \rightarrow L^{2}\left(0, T ;\left(L^{2}(\Omega)\right)^{3}\right),
$$

que é uniformemente contínua. De agora em diante, denotaremos $g\left(t, u_{t}\right)=\widetilde{G}(u)(t)$ para $u \in L^{2}(-h, T ; V)$, e então a hipótese (H4) torna-se verdadeira para $u, v \in L^{2}(-h, T ; V)$.

Vamos definir $F(t, \psi)=\psi(-\tau(t))$, onde $\tau \in C^{1}([0,+\infty) ;[0, h]), \tau(t) \geq 0, \tau^{\prime}(t) \leq \tau^{*}<$ 1 , para todo $t>0$. Note que neste caso $F\left(t, u_{t}\right)=u_{t}(-\tau(t))=u(t-\tau(t))$.

Estamos interessados no seguinte problema

$$
\begin{aligned}
\left\langle\frac{d u(t)}{d t}, v\right\rangle+\nu(\nabla u(t), \nabla v) & +b\left(F\left(t, u_{t}\right), u(t), v\right) \\
& =\langle f(t), v\rangle+\left(g\left(t, u_{t}\right), v\right) \quad \forall v \in V, \\
u(0) & =u_{0}, \\
u(t) & =\phi(t) \quad t \in(-h, 0),
\end{aligned}
$$

onde $u_{0} \in H, \phi \in L^{2}(-h, 0 ; V)$ e $f \in L^{2}\left(0, T ; V^{\prime}\right)$.

Definimos, o operador $A: V \rightarrow V^{\prime}$ por

$$
\langle A u, v\rangle=\int_{\Omega} \nabla u \cdot \nabla v d x=\langle\nabla u, \nabla v\rangle_{\left(L^{2}(\Omega)\right)^{3}}=\langle u, v\rangle_{V},
$$

com domínio $D(A)=\left(H^{2}(\Omega)\right)^{3} \cap V$. Então

$$
A u=-P \Delta u \quad \forall u \in D(A),
$$

onde $P$ é a projeção ortogonal de $\left(L^{2}(\Omega)\right)^{3}$ em $H$. Além disso, podemos definir o operador $B: V \times V \rightarrow V^{\prime}$ por

$$
\langle B(u, v), w\rangle=b(u, v, w) .
$$

Então o problema (3.3) pode ser reescrito na forma

$$
\begin{aligned}
\frac{d u(t)}{d t}+\nu A u(t)+B\left(F\left(t, u_{t}\right), u(t)\right) & =f(t)+g\left(t, u_{t}\right), \quad t \geq 0 \\
u(0) & =u_{0}, \\
u(t) & =\phi(t), \quad t \in(-h, 0) .
\end{aligned}
$$

\subsection{Existência de soluções}

Teorema 3.2.1. Seja $u_{0} \in H, \phi \in L^{2}(-h, 0 ; V), f \in L^{2}\left(0, T ; V^{\prime}\right)$ e assuma que $g:[0, T] \times$ $C([-h, 0] ; V) \rightarrow\left(L^{2}(\Omega)\right)^{3}$ satisfaz as hipóteses (H1)-(H5). Então, para cada $T>0$ fixo, existe pelo menos uma solução fraca $u$, para o problema (3.4), tal que $u \in L^{\infty}(0, T ; H) \cap L^{2}(0, T ; V)$. 
Prova. Provaremos a existência de solução fraca para o problema (3.4) usando o método de Faedo-Galerkin. Consideremos as auto funções $\left\{w_{j}\right\}$ do operador de Stokes em $\Omega$ com condições de fronteira de Dirichlet homogêneas. Com a ajuda destas auto funções, definimos o seguinte problema aproximado de ordem $k \geq 1$ :

$$
\text { encontrar } \quad u^{k} \quad \operatorname{com} \quad u^{k}(t)=\sum_{j=1}^{k} \gamma_{j k}(t) w_{j} \in V_{k}=\operatorname{span}\left\{w_{1}, \ldots, w_{k}\right\} \text {, }
$$

tal que

$$
\begin{aligned}
\frac{d}{d t}\left\langle u^{k}(t), w_{j}\right\rangle_{L^{2}}+\nu & \left\langle A u^{k}(t), w_{j}\right\rangle+b\left(F\left(t, u_{t}^{k}\right), u^{k}(t), w_{j}\right), \\
& =\left\langle f(t), w_{j}\right\rangle+\left\langle g\left(t, u_{t}^{k}\right), w_{j}\right\rangle_{L^{2}} \quad 1 \leq j \leq k \\
u^{k}(0) & =P_{k} u^{0}, \\
u^{k}(t) & =P_{k} \phi(t) \quad t \in(-h, 0)
\end{aligned}
$$

onde $P_{k}$ é a projeção de $H$ em $V_{k}$. Agora, (3.5) é um sistema não linear de equações diferenciais funcionais nas funções $\gamma_{1 k}, \ldots, \gamma_{k k}$. Podemos garantir existência e unicidade de solução para este problema usando o mesmo método usado por Caraballo [2]. Isto será feito no Apêndice ao final deste capítulo. Vamos mostrar que este problema pode ser colocado nas hipóteses do teorema 3.4.3. Substituindo $u^{k}(t)=\sum_{i=1}^{k} \gamma_{i k}(t) w_{i}$ em (3.5), teremos

$$
\begin{aligned}
\sum_{i=1}^{k} \frac{d}{d t} \gamma_{i k}(t)\left\langle w_{i}, w_{j}\right\rangle & +\nu \sum_{i=1}^{k} \gamma_{i k}(t)\left\langle A w_{i}, w_{j}\right\rangle \\
& +\sum_{i, l=1}^{k} \gamma_{i k}(t-\tau(t)) \gamma_{l k}(t) b\left(w_{i}, w_{l}, w_{j}\right) \\
& =\left\langle f(t), w_{j}\right\rangle+\left\langle g\left(t, u_{t}^{k}\right), w_{j}\right\rangle
\end{aligned}
$$

para $j=1, \ldots, k$. Escrevendo este sistema em notação matricial e levando em conta que a matriz $\left[\left\langle w_{i}, w_{j}\right\rangle\right]_{i, j=1, \ldots, k}$ é a matriz identidade, temos a equação matricial

$$
\begin{aligned}
{\left[\begin{array}{c}
\frac{d}{d t} \gamma_{1 k}(t) \\
\frac{d}{d t} \gamma_{2 k}(t) \\
\vdots \\
\frac{d}{d t} \gamma_{k k}(t)
\end{array}\right]+\nu\left[\begin{array}{c}
\sum_{i=1}^{k} \gamma_{i k}(t)\left\langle A w_{i}, w_{1}\right\rangle \\
\sum_{i=1}^{k} \gamma_{i k}(t)\left\langle A w_{i}, w_{2}\right\rangle \\
\vdots \\
\sum_{i=1}^{k} \gamma_{i k}(t)\left\langle A w_{i}, w_{k}\right\rangle
\end{array}\right]+\left[\begin{array}{c}
\sum_{i, l=1}^{k} \gamma_{i k}(t-\tau(t)) \gamma_{l k}(t) b\left(w_{i}, w_{l}, w_{1}\right) \\
\sum_{i, l=1}^{k} \gamma_{i k}(t-\tau(t)) \gamma_{l k}(t) b\left(w_{i}, w_{l}, w_{2}\right) \\
\vdots \\
\sum_{i, l=1}^{k} \gamma_{i k}(t-\tau(t)) \gamma_{l k}(t) b\left(w_{i}, w_{l}, w_{k}\right)
\end{array}\right] } \\
=\left[\begin{array}{c}
\left\langle f(t), w_{1}\right\rangle \\
\left\langle f(t), w_{2}\right\rangle \\
\vdots \\
\left\langle f(t), w_{k}\right\rangle
\end{array}\right]+\left[\begin{array}{c}
\left\langle g\left(t, u_{t}^{k}\right), w_{1}\right\rangle \\
\left\langle g\left(t, u_{t}^{k}\right), w_{2}\right\rangle \\
\vdots \\
\left\langle g\left(t, u_{t}^{k}\right), w_{k}\right\rangle
\end{array}\right],
\end{aligned}
$$


que escreveremos na forma simplificada

$$
\frac{d}{d t} \Gamma_{k}(t)=\Phi_{1}\left(\Gamma_{k}(t)\right)+\Phi_{2}\left(\Gamma_{k}(t-\tau(t)), \Gamma_{k}(t)\right)+\Phi_{3}(f(t))+\Phi_{4}\left(g\left(t, u_{t}^{k}\right)\right),
$$

onde as funções $\Phi_{1}, \Phi_{2}, \Phi_{3}$ e $\Phi_{4}$, são funções vetoriais dadas como sugere a equação matricial, e $\Gamma_{k}(t)=\left(\gamma_{1 k}(t), \ldots, \gamma_{k k}(t)\right)$. Vamos mostrar que $\Phi_{i},(i=1,2,3,4)$, satisfazem as hipóteses (3.21)-(3.25). Denotemos $\|\cdot\|_{k}$ a norma em $\mathbb{R}^{k}$ que, em virtude da equivalência das normas em $\mathbb{R}^{k}$, podemos considerar como a norma da soma. Dadas $\Gamma_{k}(t)$ e $\bar{\Gamma}_{k}(t)$ em $\mathbb{R}^{k}$, temos para $\Phi_{1}$,

$$
\begin{aligned}
\| \Phi_{1}\left(\Gamma_{k}(t)\right) & -\Phi_{1}\left(\bar{\Gamma}_{k}(t)\right) \|_{k} \\
& =\nu \sum_{j=1}^{k}\left|\sum_{i=1}^{k} \gamma_{i k}(t)\left\langle A w_{i}, w_{j}\right\rangle-\sum_{i=1}^{k} \bar{\gamma}_{i k}(t)\left\langle A w_{i}, w_{j}\right\rangle\right| \\
& \leq \nu \sum_{j=1}^{k}\left(\sum_{i=1}^{k}\left|\left\langle A w_{i}, w_{j}\right\rangle \| \gamma_{i k}(t)-\bar{\gamma}_{i k}(t)\right|\right) \\
& \leq \nu\left(\sum_{j=1}^{k} \max _{1 \leq i \leq k}\left|\left\langle A w_{i}, w_{j}\right\rangle\right|\right) \sum_{i=1}^{k}\left|\gamma_{i k}(t)-\bar{\gamma}_{i k}(t)\right| \\
& =\mathcal{C}_{1}\left\|\Gamma_{k}(t)-\bar{\Gamma}_{k}(t)\right\|_{k} .
\end{aligned}
$$

Para a função $\Phi_{2}$ temos

$$
\begin{aligned}
\| \Phi_{2}( & \left.\Gamma_{k}(t-\tau(t)), \Gamma_{k}(t)\right)-\Phi_{2}\left(\bar{\Gamma}_{k}(t-\tau(t)), \bar{\Gamma}_{k}(t)\right) \|_{k} \\
& =\sum_{j=1}^{k}\left|\sum_{i, l=1}^{k} \gamma_{i k}(t-\tau(t)) \gamma_{l k}(t) b\left(w_{i}, w_{l}, w_{j}\right)-\sum_{i, l=1}^{k} \bar{\gamma}_{i k}(t-\tau(t)) \bar{\gamma}_{l k}(t) b\left(w_{i}, w_{l}, w_{j}\right)\right| \\
& \leq\left(\sum_{j=1}^{k} \max _{1 \leq i, l \leq k}\left|b\left(w_{i}, w_{l}, w_{j}\right)\right|\right) \sum_{i, l=1}^{k}\left|\gamma_{i k}(t-\tau(t)) \gamma_{l k}(t)-\bar{\gamma}_{i k}(t-\tau(t)) \bar{\gamma}_{l k}(t)\right| \\
& =\mathcal{C}_{2} \sum_{i, l=1}^{k}\left|\gamma_{i k}(t-\tau(t)) \gamma_{l k}(t)-\bar{\gamma}_{i k}(t-\tau(t)) \bar{\gamma}_{l k}(t)\right| \\
& =\mathcal{C}_{2} \sum_{i, l=1}^{k}\left|\left[\gamma_{i k}(t-\tau(t))-\bar{\gamma}_{i k}(t-\tau(t))\right] \gamma_{l k}(t)+\bar{\gamma}_{i k}(t-\tau(t))\left[\gamma_{l k}(t)-\bar{\gamma}_{l k}(t)\right]\right| \\
& \leq \mathcal{C}_{2}\left[\sum_{i, l=1}^{k}\left|\gamma_{i k}(t-\tau(t))-\bar{\gamma}_{i k}(t-\tau(t))\right|\left|\gamma_{l k}(t)\right|\right. \\
\leq & \mathcal{C}_{2}\left[\sum_{i=1}^{k}\left|\gamma_{i k}(t-\tau(t))-\bar{\gamma}_{i k}(t-\tau(t))\right| \sum_{l=1}^{k}\left|\gamma_{l k}(t)\right|\right.
\end{aligned}
$$




$$
\begin{gathered}
\left.+\sum_{i=1}^{k}\left|\bar{\gamma}_{i k}(t-\tau(t))\right| \sum_{l=1}^{k}\left|\gamma_{l k}(t)-\bar{\gamma}_{l k}(t)\right|\right] \\
=\mathcal{C}_{2}\left(\| \Gamma_{k}(t-\right. \\
\tau(t))-\bar{\Gamma}_{k}(t-\tau(t))\left\|_{k}\right\| \Gamma_{k}(t) \|_{k} \\
\left.+\left\|\bar{\Gamma}_{k}(t-\tau(t))\right\|_{k}\left\|\Gamma_{k}(t)-\bar{\Gamma}_{k}(t)\right\|_{k}\right)
\end{gathered}
$$

Para $\Phi_{3}$ temos a limitação

$$
\begin{aligned}
\left\|\Phi_{3}(f(t))\right\|_{k} & =\sum_{j=1}^{k}\left|\left\langle f(t), w_{j}\right\rangle\right| \\
& \leq \sum_{j=1}^{k}\|f(t)\|_{V^{\prime}}\left\|w_{j}\right\|_{V} \\
& =\left(\sum_{j=1}^{k}\left\|w_{j}\right\|_{V}\right)\|f(t)\|_{V^{\prime}}=\mathcal{C}_{3}\|f(t)\|_{V^{\prime}} .
\end{aligned}
$$

A função vetorial $\Phi_{4}$ por sua vez é limitada em termos de $g$. De fato,

$$
\begin{aligned}
\| \Phi_{4}\left(g\left(t, u_{t}^{k}\right)\right) & -\Phi_{4}\left(g\left(t, v_{t}^{k}\right)\right) \|_{k} \\
& =\sum_{j=1}^{k}\left|\left\langle g\left(t, u_{t}^{k}\right), w_{j}\right\rangle-\left\langle g\left(t, v_{t}^{k}\right), w_{j}\right\rangle\right| \\
& =\sum_{j=1}^{k}\left|\left\langle g\left(t, u_{t}^{k}\right)-g\left(t, v_{t}^{k}\right), w_{j}\right\rangle\right| \\
& \leq\left(\sum_{j=1}^{k}\left\|w_{j}\right\|_{L^{2}}\right)\left\|g\left(t, u_{t}^{k}\right)-g\left(t, v_{t}^{k}\right)\right\|_{L^{2}}=\mathcal{C}_{4}\left\|g\left(t, u_{t}^{k}\right)-g\left(t, v_{t}^{k}\right)\right\|_{L^{2}} .
\end{aligned}
$$

Notemos ainda que para $\Gamma_{k}(t)=\left(\gamma_{1 k}(t), \gamma_{2 k}(t), \ldots, \gamma_{k k}(t)\right)=0$, teremos

$$
u^{k}(t)=\sum_{j=1}^{k} \gamma_{j k}(t) w_{j}=0,
$$

e portanto $g\left(t, u_{t}^{k}\right)=0$ e $\Phi_{1}(0)=\Phi_{2}(0,0)=\Phi_{4}(0)=0$. Além disso, considerando $W=$ $\left(w_{1}, w_{2}, \ldots, w_{k}\right)$, temos

$$
\begin{aligned}
\left\|u^{k}(s)-v^{k}(s)\right\|_{V}^{2} & =\left\|\nabla u^{k}(s)-\nabla v^{k}(s)\right\|_{L^{2}}^{2} \\
& =\left\|\sum_{j=1}^{k}\left(\gamma_{j k}(s) \nabla w_{j}-\bar{\gamma}_{j k}(s) \nabla w_{j}\right)\right\|_{L^{2}}^{2} \\
& =\left\|\left\langle\Gamma_{k}(s)-\bar{\Gamma}_{k}(s), \nabla W\right\rangle_{k}\right\|_{L^{2}}^{2} \\
& \leq\|\| \Gamma_{k}(s)-\bar{\Gamma}_{k}(s)\left\|_{k}\right\| \nabla W\left\|_{k}\right\|_{L^{2}}^{2} \\
& =\int_{\Omega}\left\|\Gamma_{k}(s)-\bar{\Gamma}_{k}(s)\right\|_{k}^{2}\|\nabla W\|_{k}^{2} d x
\end{aligned}
$$




$$
\begin{aligned}
& =\left\|\Gamma_{k}(s)-\bar{\Gamma}_{k}(s)\right\|_{k}^{2} \int_{\Omega}\|\nabla W\|_{k}^{2} d x \\
& \leq\left\|\Gamma_{k}(s)-\bar{\Gamma}_{k}(s)\right\|_{k}^{2}\|W\|_{V}^{2} \\
& =\left\|\Gamma_{k}(s)-\bar{\Gamma}_{k}(s)\right\|_{k}^{2} \sum_{j=1}^{k}\left\|w_{j}\right\|_{V}^{2}=\mathcal{C}_{5}\left\|\Gamma_{k}(s)-\bar{\Gamma}_{k}(s)\right\|_{k}^{2} .
\end{aligned}
$$

As hipóteses do teorema 3.4.3 são satisfeitas e a sua conclusão nos garante que o problema (3.5) tem solução única maximal definida no intervalo $\left[0, t_{k}\right)$ com $0<t_{k} \leq T$.

As estimativas a priori que iremos obter mostrarão que $t_{k}=T$. Multiplicando cada equação em (3.5) pela respectiva função $\gamma_{j k}(t)$, e somando em $j$, obtemos

$$
\begin{gathered}
\frac{1}{2} \frac{d}{d t}\left\|u^{k}(t)\right\|_{L^{2}}^{2}+\nu\left\|\nabla u^{k}(t)\right\|_{L^{2}}^{2}+\int_{\Omega} F\left(t, u_{t}^{k}\right) \nabla u^{k}(t) u^{k}(t) d x \\
=\left\langle f(t), u^{k}(t)\right\rangle+\left\langle g\left(t, u_{t}^{k}\right), u^{k}(t)\right\rangle .
\end{gathered}
$$

O termo não linear é tratado de forma usual

$$
\begin{aligned}
\int_{\Omega} F\left(t, u_{t}^{k}\right) \nabla u^{k}(t) u^{k}(t) d x & =\int_{\Omega} F\left(t, u_{t}^{k}\right) \nabla\left(\frac{\left|u^{k}(t)\right|^{2}}{2}\right) d x \\
& =-\int_{\Omega} \operatorname{div} F\left(t, u_{t}^{k}\right)\left(\frac{\left|u^{k}(t)\right|^{2}}{2}\right) d x=0,
\end{aligned}
$$

onde usamos que $u^{k}=0$ sobre a fronteira e a condição de divergência livre sobre $u^{k}$ para concluir que $\operatorname{div} F\left(t, u_{t}^{k}\right)=\operatorname{div} u^{k}(t-\tau(t))=0$.

Agora, integrando em $t$ e usando as desigualdades de Hölder e Young encontramos

$$
\begin{aligned}
\left\|u^{k}(t)\right\|_{L^{2}}^{2}+2 \nu & \int_{0}^{t}\left\|u^{k}(s)\right\|_{V}^{2} d s \\
\leq & \left\|u^{0}\right\|_{L^{2}}^{2}+\frac{1}{\nu} \int_{0}^{t}\|f(s)\|_{V^{\prime}}^{2} d s+\nu \int_{0}^{t}\left\|u^{k}(s)\right\|_{V}^{2} d s \\
& \quad+\frac{\nu}{2 C_{g}} \int_{0}^{t}\left\|g\left(s, u_{s}^{k}\right)\right\|_{L^{2}}^{2} d s+\frac{2 C_{g}}{\nu} \int_{0}^{t}\left\|u^{k}(s)\right\|_{L^{2}}^{2} d s .
\end{aligned}
$$

Das hipóteses (H2) e (H4), podemos limitar o termo de retardo da seguinte forma

$$
\begin{aligned}
\frac{\nu}{2 C_{g}} \int_{0}^{t}\left\|g\left(s, u_{s}^{k}\right)\right\|_{L^{2}}^{2} d s & \leq \frac{C_{g} \nu}{2 C_{g}} \int_{-h}^{t}\left\|u^{k}(s)\right\|_{V}^{2} d s \\
& \leq \frac{\nu}{2} \int_{-h}^{0}\|\phi(s)\|_{V}^{2} d s+\frac{\nu}{2} \int_{0}^{t}\left\|u^{k}(s)\right\|_{V}^{2} d s .
\end{aligned}
$$

Portanto

$$
\begin{aligned}
\left\|u^{k}(t)\right\|_{L^{2}}^{2}+\frac{\nu}{2} \int_{0}^{t}\left\|u^{k}(s)\right\|_{V}^{2} d s \leq & \left\|u^{0}\right\|_{L^{2}}^{2}+\frac{1}{\nu} \int_{0}^{t}\|f(s)\|_{V^{\prime}}^{2} d s \\
& +\frac{\nu}{2} \int_{-h}^{0}\|\phi(s)\|_{V^{2}}^{2} d s+\frac{2 C_{g}}{\nu} \int_{0}^{t}\left\|u^{k}(s)\right\|_{L^{2}}^{2} d s .
\end{aligned}
$$


Após descarte no primeiro membro usamos desigualdade de Gronwall para garantir que $\left\|u^{k}(t)\right\|_{L^{2}}$ é limitada independentemente de $k$. Esta estimativa nos permite tomar $t_{k}=T$ e então a sequência $u^{k}$ é limitada em $L^{\infty}(0, T ; H)$ e em $L^{2}(0, T ; V)$, independentemente de $k$. Em particular, a sequência de funções $F\left(\cdot, u^{k}\right)$ é limitada em $L^{2}(-h, T ; V)$.

Vamos agora estimar $\frac{d}{d t} u^{k}(t)$ em $L^{r}\left(0, T ; V^{\prime}\right)$ para algum $r>1$. Das equações (3.5), temos que,

$$
\frac{d u^{k}(t)}{d t}+\nu A u^{k}(t)+B\left(F\left(t, u_{t}^{k}\right), u^{k}(t)\right)=f(t)+g\left(t, u_{t}^{k}\right),
$$

em $V^{\prime}$. Dada então $v \in V$ de forma que $\|v\|_{V} \leq 1$, temos

$$
\begin{aligned}
&\left\langle\frac{d}{d t} u^{k}(t), v\right\rangle=\left\langle-\nu A u^{k}(t)-B\left(F\left(t, u_{t}^{k}\right), u^{k}(t)\right)+f(t)+g\left(t, u_{t}^{k}\right), v\right\rangle \\
&=-\nu\left\langle\nabla u^{k}(t), \nabla v\right\rangle-b\left(F\left(t, u_{t}^{k}\right), u^{k}(t), v\right)+\langle f(t), v\rangle+\left\langle g\left(t, u_{t}^{k}\right), v\right\rangle \\
& \leq \nu\left\|u^{k}(t)\right\|_{V}\|v\|_{V}+\left|b\left(F\left(t, u_{t}^{k}\right), u^{k}(t), v\right)\right| \\
& \quad+\|f(t)\|_{V^{\prime}}\|v\|_{V}+\left\|g\left(t, u_{t}^{k}\right)\right\|_{L^{2}}\|v\|_{V} .
\end{aligned}
$$

Como a sequência $u^{k}$ é limitada em $L^{2}(0, T ; V), f(t) \in L^{2}\left(0, T ; V^{\prime}\right)$ e $g$ satisfaz (H4), então ainda temos que limitar o termo trilinear. Este por sua vez pode ser estimado usando Hölder e desigualdades de interpolação. De fato, para qualquer $v \in V$,

$$
\begin{aligned}
\left|\int_{\Omega} F\left(t, u_{t}^{k}\right) \nabla u^{k} v d x\right| & =\left|-\int_{\Omega} F\left(t, u_{t}^{k}\right) \nabla v u^{k} d x\right| \\
& \leq\left\|F\left(t, u_{t}^{k}\right)\right\|_{L^{4}}\|\nabla v\|_{L^{2}}\left\|u^{k}\right\|_{L^{4}} \\
& \leq C\left\|F\left(t, u_{t}^{k}\right)\right\|_{L^{4}}\left\|u^{k}\right\|_{V}^{\frac{3}{4}}\left\|u^{k}\right\|_{L^{2}}^{\frac{1}{4}}\|v\|_{V} .
\end{aligned}
$$

Como $V \hookrightarrow\left(L^{4}(\Omega)\right)^{3}$, e $u^{k}$ é limitada em $L^{\infty}(0, T ; H)$, então temos

$$
\left|\int_{\Omega} F\left(t, u_{t}^{k}\right) \nabla u^{k} v d x\right| \leq C\left\|F\left(t, u_{t}^{k}\right)\right\|_{V}\left\|u^{k}\right\|_{V}^{\frac{3}{4}}\|v\|_{V}
$$

Desta forma temos que

$$
\left\|B\left(F\left(\cdot, u_{\cdot}^{k}\right), u^{k}\right)\right\|_{V^{\prime}} \leq C\left\|F\left(t, u_{t}^{k}\right)\right\|_{V}\left\|u^{k}\right\|_{V}^{\frac{3}{4}}
$$

e então, integrando em $t$, e usando a desigualdade de Hölder com $p=\frac{7}{3}$ e $q=\frac{7}{4}$, temos

$$
\begin{aligned}
\int_{0}^{t}\left\|B\left(F\left(s, u_{s}^{k}\right), u^{k}\right)\right\|_{V^{\prime}}^{\frac{8}{7}} d s & \leq C \int_{0}^{t}\left\|F\left(s, u_{s}^{k}\right)\right\|_{V}^{\frac{8}{7}}\left\|u^{k}(s)\right\|_{V}^{\frac{6}{7}} d s \\
& \leq \frac{4 C}{7} \int_{0}^{t}\left\|F\left(s, u_{s}^{k}\right)\right\|_{V}^{2} d s+\frac{3}{7} \int_{0}^{t}\left\|u^{k}(s)\right\|_{V}^{2} d s
\end{aligned}
$$

donde segue que $B\left(F\left(\cdot, u^{k}\right), u^{k}\right)$ é limitado em $L^{\frac{8}{7}}\left(0, T ; V^{\prime}\right)$, e $\frac{d}{d t} u^{k}(t)$ é então limitada em $L^{\frac{8}{7}}\left(0, T ; V^{\prime}\right)$. 
Então, pelo teorema de compacidade de Aubin-Lions, existe uma subsequência de $u^{k}$ (que será ainda denotada por $\left.u^{k}\right)$ e uma função $u \in L^{2}(-h, T ; V)$ tal que

$$
u^{k} \rightarrow u \quad \text { fortemente em } \quad L^{2}(-h, T ; H) .
$$

Além disso, segue da limitação de $u^{k}$ em $L^{2}(-h, T ; V)$ que

$$
u^{k} \rightarrow u \quad \text { fracamente em } \quad L^{2}(-h, T ; V)
$$

Da convergência forte de $u^{k}$ para $u$ e de $\phi^{k}=P_{k} \phi$ para $\phi$, temos que

$$
F\left(\cdot, u_{.}^{k}\right) \rightarrow F(\cdot, u .) \quad \text { fortemente em } \quad L^{2}(0, T ; H) .
$$

Finalmente da condição $(H 5)$ segue imediatamente que

$$
g\left(\cdot, u_{.}^{k}\right) \rightarrow g(\cdot, u .) \quad \text { fracamente em } \quad L^{2}\left(0, T ; V^{\prime}\right) .
$$

Consequentemente, passando o limite em (3.7), temos que $u(t)$ é uma solução fraca do problema (3.4). A prova está completa.

A seguir discutiremos a existência e unicidade de soluções fortes para o problema (3.4). Vamos considerar que $g$ é dada na forma

$$
g\left(t, u_{t}\right)=G(u(t-\rho(t)))
$$

com $G: \mathbb{R}^{3} \rightarrow \mathbb{R}^{3}$ uma função Lipschitz contínua com constante de Lipschitz $L$ satisfazendo $G(0)=0$ e $\rho \in C^{1}([0, \infty) ;[0, h]), \rho(t) \geq 0$, e $\rho^{\prime}(t) \leq \rho^{*}<1$, para todo $t \geq 0$. É fácil ver que nestas condições $g$ satisfaz as hipóteses (H1)-(H3). Além disso, usando mudança de variáveis $\eta=s-\rho(s)$ temos

$$
\begin{aligned}
\int_{0}^{t} \| g\left(s, u_{s}\right) & -g\left(s, v_{s}\right) \|_{L^{2}}^{2} d s \\
& =\int_{0}^{t}\|G(u(s-\rho(s)))-G(v(s-\rho(s)))\|_{L^{2}}^{2} d s \\
& =\int_{0}^{t} \int_{\Omega}\|G(u(s-\rho(s)))-G(v(s-\rho(s)))\|_{\mathbb{R}^{3}}^{2} d x d s \\
& =\int_{0}^{t} \int_{\Omega} L^{2}\|u(s-\rho(s))-v(s-\rho(s))\|_{\mathbb{R}^{3}}^{2} d x d s \\
& =L^{2} \int_{0}^{t}\|u(s-\rho(s))-v(s-\rho(s))\|_{L^{2}}^{2} d s \\
& \leq L^{2} \int_{-\rho(0)}^{t-\rho(t)} \frac{1}{1-\rho^{*}}\|u(\eta)-v(\eta)\|_{L^{2}}^{2} d \eta \\
& \leq \frac{L^{2}}{1-\rho^{*}} \int_{-h}^{t}\|u(s)-v(s)\|_{L^{2}}^{2} d s,
\end{aligned}
$$

e consequentemente (H4) e (H5) também ocorrem, com constante $C_{g}$ igual a $\frac{L^{2}}{1-\rho^{*}}$. Também vamos supor que $f$ satisfaz uma hipótese como em Taniguchi [25, Teorema 5.2], 
(Hf) $\|f(s)\|_{L^{2}}^{2}$ é uniformemente $L(\theta)$-integrável, isto é,

$$
\int_{0}^{t} e^{\theta(s-t)}\|f(s)\|_{L^{2}}^{2} d s \leq L(\theta)
$$

Teorema 3.2.2. Sejam $u^{0} \in V, \phi \in L^{\infty}(-h, 0 ; V), f \in L^{2}\left(0, T ;\left(L^{2}(\Omega)\right)^{3}\right)$, e $g:[0, T] \times$ $C([-h, 0] ; V) \rightarrow\left(L^{2}(\Omega)\right)^{3}$ satisfazendo as hipóteses (H1)-(H5). Suponha ainda que (1 $\left.\rho^{*}\right) \nu^{2} \lambda_{1}^{2}>4 L$, sendo $\lambda_{1}$ o primeiro autovalor do operador $A$, e sejam, $\kappa>0$ um número real tal que

$$
0<\kappa<\frac{\left(1-\rho^{*}\right) \nu^{4} \lambda_{1}^{2}-4 L \nu^{2}}{27\left(1-\rho^{*}\right) \lambda_{1} c_{3}^{4}}
$$

$e \theta^{*}>0$ tal que

$$
\frac{\nu}{2}-\frac{\theta^{*}}{\lambda_{1}}-\frac{27 c_{3}^{4} \kappa}{2 \nu^{3} \lambda_{1}}-\frac{2 e^{h \theta^{*}} L}{\left(1-\rho^{*}\right) \nu \lambda_{1}^{2}}=0 .
$$

Suponha ainda $f(t)$ satisfazendo a hipótese (Hf) $e$

$$
\|\phi\|_{L^{\infty}(-h, 0 ; V)}^{2}+\frac{2 L^{2}}{\nu\left(1-\rho^{*}\right)}\|\phi\|_{L^{2}(-h, 0 ; H)}^{2}+\left\|u^{0}\right\|_{V}^{2}+\frac{2}{\nu} L(\theta)<\sqrt{\kappa} .
$$

Então, para cada $T>0$ fixado, existe uma única solução forte u para o problema (3.4) tal que $u \in L^{\infty}(0, T ; V) \cap L^{2}(0, T ; D(A))$ satisfazendo

$$
\|u(t)\|_{V}^{2} \leq\left(\left\|u^{0}\right\|_{V}^{2}+\frac{2 L^{2}}{\nu\left(1-\rho^{*}\right)}\|\phi\|_{L^{2}(-h, 0 ; H)}^{2}\right) e^{-\theta t}+\frac{2}{\nu} L(\theta),
$$

para algum $\theta \in\left(0, \theta^{*}\right)$. Mais ainda, se $\phi \in C([-h, 0] ; V)$ e $\phi(0)=u^{0}$ então $u \in C([-h, T] ; V)$.

Prova. Definimos $W\left(t, u^{k}\right)$ da forma,

$$
W\left(t, u^{k}\right)=e^{\theta t}\left\|u^{k}(t)\right\|_{V}^{2}+\frac{2}{\nu\left(1-\rho^{*}\right)} \int_{t-\rho(t)}^{t} e^{\theta s} e^{\theta h}\left\|G\left(u^{k}(s)\right)\right\|_{L^{2}}^{2} d s,
$$

onde $\theta>0$ ainda será escolhido. Vamos mostrar que nestas condições $\left\|u^{k}(t)\right\|_{V}<\sqrt[4]{\kappa}$, para todo $0 \leq t \leq T$. Suponha, por absurdo, a existência de $t_{*} \in[0, T]$ tal que $\left\|u^{k}\left(t_{*}\right)\right\|_{V}=\sqrt[4]{\kappa}$. Mais ainda, suponha que $t_{*}$ seja o menor valor tal que isto aconteça, isto é, $\left\|u^{k}(t)\right\|_{V}<\sqrt[4]{\kappa}$ para todo $0 \leq t<t_{*}$. Então para todo $0 \leq t \leq t_{*}$, temos

$$
\begin{aligned}
& \frac{d}{d t} W\left(t, u^{k}\right) \\
& =\theta e^{\theta t}\left\|u^{k}(t)\right\|_{V}^{2}+e^{\theta t} \frac{d}{d t}\left\|u^{k}(t)\right\|_{V}^{2}+\frac{2}{\left(1-\rho^{*}\right) \nu} e^{\theta t} e^{\theta h}\left\|G\left(u^{k}(t)\right)\right\|_{L^{2}}^{2} \\
& \quad-\frac{2 e^{\theta(t-\rho(t))} e^{\theta h}\left(1-\rho^{\prime}(t)\right)}{\nu\left(1-\rho^{*}\right)}\left\|G\left(u^{k}(t-\rho(t))\right)\right\|_{L^{2}}^{2} \\
& \leq \theta e^{\theta t}\left\|u^{k}(t)\right\|_{V}^{2}+2 e^{\theta t}\left\langle A u^{k}(t), \frac{d}{d t} u^{k}(t)\right\rangle \\
& \quad+\frac{2 e^{\theta h} L e^{\theta t}}{\left(1-\rho^{*}\right) \nu}\left\|u^{k}(t)\right\|_{L^{2}}^{2}-\frac{2 e^{\theta t}}{\nu} e^{\theta(h-\rho(t))} \frac{\left(1-\rho^{\prime}(t)\right)}{1-\rho^{*}}\left\|G\left(u^{k}(t-\rho(t))\right)\right\|_{L^{2}}^{2}
\end{aligned}
$$




$$
\begin{aligned}
\leq \theta e^{\theta t} & \left\|u^{k}(t)\right\|_{V}^{2} \\
& +2 e^{\theta t}\left\langle A u^{k}(t),-\nu A u^{k}(t)-B\left(F\left(t, u_{t}^{k}\right), u^{k}(t)\right)+f(t)+G\left(u^{k}(t-\rho(t))\right)\right\rangle \\
& +\frac{2 e^{\theta h} L e^{\theta t}}{\left(1-\rho^{*}\right) \nu}\left\|u^{k}(t)\right\|_{L^{2}}^{2}-\frac{2 e^{\theta t}}{\nu}\left\|G\left(u^{k}(t-\rho(t))\right)\right\|_{L^{2}}^{2} .
\end{aligned}
$$

Mas notemos que

$$
2 e^{\theta t}\left\langle A u^{k}(t),-\nu A u^{k}(t)\right\rangle=-2 \nu e^{\theta t}\left\|A u^{k}(t)\right\|_{L^{2}}^{2},
$$

e

$$
\begin{aligned}
2 e^{\theta t}\left\langle A u^{k}(t),\right. & \left.-B\left(F\left(t, u_{t}^{k}\right), u^{k}(t)\right)\right\rangle \\
& =-2 e^{\theta t}\left\langle B\left(F\left(t, u_{t}^{k}\right), u^{k}(t)\right), A u^{k}(t)\right\rangle \\
& =-2 e^{\theta t} b\left(F\left(t, u_{t}^{k}\right), u^{k}(t), A u^{k}(t)\right) \\
& \leq e^{\theta t} 2 C_{3}\left\|F\left(t, u_{t}^{k}\right)\right\|_{V}\left\|u^{k}(t)\right\|_{V}^{\frac{1}{2}}\left\|A u^{k}(t)\right\|_{L^{2}}^{\frac{1}{2}}\left\|A u^{k}(t)\right\|_{L^{2}} \\
& =e^{\theta t} 2 C_{3}\left\|F\left(t, u_{t}^{k}\right)\right\|_{V}\left\|u^{k}(t)\right\|_{V}^{\frac{1}{2}}\left\|A u^{k}(t)\right\|_{L^{2}}^{\frac{3}{2}}\left(\frac{4 \nu}{3 \cdot 2}\right)^{\frac{3}{4}}\left(\frac{3 \cdot 2}{4 \nu}\right)^{\frac{3}{4}} \\
& \leq e^{\theta t}\left(\frac{1}{4} C_{3}^{4} 2^{4}\left(\frac{3 \cdot 2}{4 \nu}\right)^{3}\left\|F\left(t, u_{t}^{k}\right)\right\|_{V}^{4}\left\|u^{k}(t)\right\|_{V}^{2}+\frac{3}{4} \frac{4 \nu}{3 \cdot 2}\left\|A u^{k}(t)\right\|_{L^{2}}^{2}\right) \\
& =\frac{27 C_{3}^{4} e^{\theta t}}{2 \nu^{3}}\left\|F\left(t, u_{t}^{k}\right)\right\|_{V}^{4}\left\|u^{k}(t)\right\|_{V}^{2}+\frac{\nu}{2} e^{\theta t}\left\|A u^{k}(t)\right\|_{L^{2}}^{2},
\end{aligned}
$$

e

$$
\begin{aligned}
2 e^{\theta t}\left\langle A u^{k}(t), f(t)\right\rangle & \leq 2 e^{\theta t}\left\|A u^{k}(t)\right\|_{L^{2}}\|f(t)\|_{L^{2}}, \\
& \leq \frac{\nu}{2} e^{\theta t}\left\|A u^{k}(t)\right\|_{L^{2}}^{2}+\frac{2}{\nu} e^{\theta t}\|f(t)\|_{L^{2}}^{2},
\end{aligned}
$$

e

$$
\begin{aligned}
2 e^{\theta t}\left\langle A u^{k}(t), G(u(t-\rho(t)))\right\rangle & \leq 2 e^{\theta t}\left\|A u^{k}(t)\right\|_{L^{2}}\|G(u(t-\rho(t)))\|_{L^{2}} \\
& \leq \frac{\nu}{2} e^{\theta t}\left\|A u^{k}(t)\right\|_{L^{2}}^{2}+\frac{2}{\nu} e^{\theta t}\left\|G\left(u^{k}(t-\rho(t))\right)\right\|_{L^{2}}^{2} .
\end{aligned}
$$

Juntando todas estas estimativas temos que

$$
\begin{aligned}
\frac{d}{d t} W\left(t, u^{k}\right) \leq \theta & e^{\theta t}\left\|u^{k}(t)\right\|_{V}^{2}-2 \nu e^{\theta t}\left\|A u^{k}(t)\right\|_{L^{2}}^{2}+\frac{\nu}{2} e^{\theta t}\left\|A u^{k}(t)\right\|_{L^{2}}^{2} \\
+ & \frac{27 C_{3}^{4} e^{\theta t}}{2 \nu^{3}}\left\|F\left(t, u_{t}^{k}\right)\right\|_{V}^{4}\left\|u^{k}(t)\right\|_{V}^{2}+\frac{\nu}{2} e^{\theta t}\left\|A u^{k}(t)\right\|_{L^{2}}^{2}+\frac{2 e^{\theta t}}{\nu}\|f(t)\|_{L^{2}}^{2} \\
+ & \frac{\nu}{2} e^{\theta t}\left\|A u^{k}(t)\right\|_{L^{2}}^{2}+\frac{2 e^{\theta h} L e^{\theta t}}{\left(1-\rho^{*}\right) \nu}\left\|u^{k}(t)\right\|_{L^{2}}^{2},
\end{aligned}
$$

e reorganizando os termos vem

$$
\begin{aligned}
\frac{d}{d t} W\left(t, u^{k}\right) \leq & \theta e^{\theta t}\left\|u^{k}(t)\right\|_{V}^{2}-\frac{\nu}{2} e^{\theta t}\left\|A u^{k}(t)\right\|_{L^{2}}^{2}+\frac{27 C_{3}^{4} e^{\theta t}}{2 \nu^{3}}\left\|F\left(t, u_{t}^{k}\right)\right\|_{V}^{4}\left\|u^{k}(t)\right\|_{V}^{2} \\
& +\frac{2 e^{\theta t}}{\nu}\|f(t)\|_{L^{2}}^{2}+\frac{2 e^{\theta h} L e^{\theta t}}{\left(1-\rho^{*}\right) \nu}\left\|u^{k}(t)\right\|_{L^{2}}^{2} .
\end{aligned}
$$


Agora como

$$
\left\|u^{k}(t)\right\|_{L^{2}}^{2} \leq \frac{1}{\lambda_{1}}\left\|u^{k}(t)\right\|_{V}^{2}
$$

temos também

$$
\begin{aligned}
\left\|u^{k}(t)\right\|_{V}^{2} & =\left\langle u^{k}(t), u^{k}(t)\right\rangle_{V}=\left\langle A u^{k}(t), u^{k}(t)\right\rangle \\
& =\int_{\Omega} \nabla u^{k}(t) \nabla u^{k}(t) d x=-\int_{\Omega} \Delta u^{k}(t) u^{k}(t) d x \\
& =\int_{\Omega}-P \Delta u^{k}(t) u^{k}(t) d x=\int_{\Omega} A u^{k}(t) u^{k}(t) d x \\
& \leq\left\|A u^{k}(t)\right\|_{L^{2}}\left\|u^{k}(t)\right\|_{L^{2}} \leq\left\|A u^{k}(t)\right\|_{L^{2}} \frac{1}{\sqrt{\lambda_{1}}}\left\|u^{k}(t)\right\|_{V},
\end{aligned}
$$

donde segue que

e portanto

$$
\left\|u^{k}(t)\right\|_{V} \leq \frac{1}{\sqrt{\lambda_{1}}}\left\|A u^{k}(t)\right\|_{L^{2}}
$$

$$
\left\|u^{k}(t)\right\|_{V}^{2} \leq \frac{1}{\lambda_{1}}\left\|A u^{k}(t)\right\|_{L^{2}}^{2}
$$

Assim,

$$
\begin{aligned}
\frac{d}{d t} W\left(t, u^{k}\right) \leq \frac{\theta}{\lambda_{1}} e^{\theta t} & \left\|A u^{k}(t)\right\|_{L^{2}}^{2}-\frac{\nu}{2} e^{\theta t}\left\|A u^{k}(t)\right\|_{L^{2}}^{2} \\
& +\frac{27 C_{3}^{4} e^{\theta t}}{2 \nu^{3} \lambda_{1}}\left\|F\left(t, u_{t}^{k}\right)\right\|_{V}^{4}\left\|A u^{k}(t)\right\|_{L^{2}}^{2} \\
& +\frac{2 e^{\theta t}}{\nu}\|f(t)\|_{L^{2}}^{2}+\frac{2 e^{\theta h} L e^{\theta t}}{\left(1-\rho^{*}\right) \nu \lambda_{1}^{2}}\left\|A u^{k}(t)\right\|_{L^{2}}^{2} .
\end{aligned}
$$

Resta apenas estimar o termo $\left\|F\left(t, u_{t}^{k}\right)\right\|_{V}^{4}$. Para isto, notemos que

$$
F\left(t, u_{t}^{k}\right)=u^{k}(t-\tau(t))= \begin{cases}\phi^{k}(t-\tau(t)) & \text { se } \quad-h \leq t-\tau(t) \leq 0 \\ u^{k}(t-\tau(t)) & \text { se } 0<t-\tau(t) \leq t_{*} .\end{cases}
$$

De qualquer forma,

$$
\left\|F\left(t, u_{t}^{k}\right)\right\|_{V}^{4} \leq \max \left\{\left\|\phi^{k}\right\|_{L^{\infty}(-h, 0 ; V)}^{4},\left\|u^{k}\right\|_{L^{\infty}\left(0, t_{*} ; V\right)}^{4}\right\} \leq \kappa
$$

Obtemos então

$$
\frac{d}{d t} W\left(t, u^{k}\right) \leq\left(\frac{\theta}{\lambda_{1}}-\frac{\nu}{2}+\frac{27 C_{3}^{4} \kappa}{2 \nu^{3} \lambda_{1}}+\frac{2 e^{\theta h} L}{\left(1-\rho^{*}\right) \nu \lambda_{1}^{2}}\right) e^{\theta t}\left\|A u^{k}(t)\right\|_{L^{2}}^{2}+\frac{2}{\nu} e^{\theta t}\|f(t)\|_{L^{2}}^{2} .
$$

Temos então que

$$
\frac{d}{d t} W\left(t, u^{k}\right)+M(\theta) e^{\theta t}\left\|A u^{k}(t)\right\|_{L^{2}}^{2} \leq \frac{2}{\nu} e^{\theta t}\|f(t)\|_{L^{2}}^{2},
$$

onde $M(\theta)=-\left(\frac{\theta}{\lambda_{1}}-\frac{\nu}{2}+\frac{27 C_{3}^{4} \kappa}{2 \nu^{3} \lambda_{1}}+\frac{2 e^{\theta h} L}{\left(1-\rho^{*}\right) \nu \lambda_{1}^{2}}\right)$. Note que para $\theta \in\left(0, \theta^{*}\right)$ temos $M(\theta)>0$. Integrando em $t$, de 0 a $t$, temos

$$
W\left(t, u^{k}\right)-W\left(0, u^{k}\right)+M(\theta) \int_{0}^{t} e^{\theta s}\left\|A u^{k}(s)\right\|_{L^{2}}^{2} d s \leq \frac{2}{\nu} \int_{0}^{t} e^{\theta s}\|f(s)\|_{L^{2}}^{2} d s,
$$


ou ainda,

$$
W\left(t, u^{k}\right)+M(\theta) \int_{0}^{t} e^{\theta s}\left\|A u^{k}(s)\right\|_{L^{2}}^{2} d s \leq W\left(0, u^{k}\right)+\frac{2 e^{\theta t}}{\nu} \int_{0}^{t} e^{\theta(s-t)}\|f(s)\|_{L^{2}}^{2} d s .
$$

Usando agora a hipótese (Hf),

$$
\begin{aligned}
e^{\theta t}\left\|u^{k}(t)\right\|_{V}^{2} & +M(\theta) \int_{0}^{t} e^{\theta s}\left\|A u^{k}(s)\right\|_{L^{2}}^{2} d s \\
& \leq\left\|u^{k}(0)\right\|_{V}^{2}+\frac{2}{\nu\left(1-\rho^{*}\right)} \int_{-\tau(0)}^{0} e^{\theta s}\left\|G\left(u^{k}(s)\right)\right\|_{L^{2}}^{2} d s+\frac{2}{\nu} e^{\theta t} L(\theta) .
\end{aligned}
$$

Como

$$
\int_{-\tau(0)}^{0} e^{\theta s}\left\|G\left(u^{k}(s)\right)\right\|_{L^{2}}^{2} d s \leq L^{2} \int_{-h}^{0}\left\|u^{k}(s)\right\|_{L^{2}}^{2} d s=L^{2} \int_{-h}^{0}\left\|\phi^{k}(s)\right\|_{L^{2}}^{2} d s
$$

e por descarte no primeiro membro, podemos então escrever

$$
e^{\theta t}\left\|u^{k}(t)\right\|_{V}^{2} \leq\left\|u^{k}(0)\right\|_{V}^{2}+\frac{2 L^{2}}{\nu\left(1-\rho^{*}\right)} \int_{-h}^{0}\left\|\phi^{k}(s)\right\|_{L^{2}}^{2} d s+\frac{2}{\nu} e^{\theta t} L(\theta),
$$

donde,

$$
\begin{aligned}
\left\|u^{k}(t)\right\|_{V}^{2} & \leq\left(\left\|u^{0}\right\|_{V}^{2}+\frac{2 L^{2}}{\nu\left(1-\rho^{*}\right)}\|\phi\|_{L^{2}(-h, 0 ; H)}^{2}\right) e^{-\theta t}+\frac{2}{\nu} L(\theta) \\
& \leq\left\|u^{0}\right\|_{V}^{2}+\frac{2 L^{2}}{\nu\left(1-\rho^{*}\right)}\|\phi\|_{L^{2}(-h, 0 ; H)}^{2}+\frac{2}{\nu} L(\theta)<\sqrt{\kappa},
\end{aligned}
$$

para todo $0 \leq t \leq t_{*}$. Isto contradiz o fato de que $\left\|u^{k}\left(t_{*}\right)\right\|=\sqrt[4]{\kappa}$. Segue que $\left\|u^{k}(t)\right\|<\sqrt[4]{\kappa}$ para todo $t \in[0, T]$, e para todo $t \in[0, T]$ temos

$$
\left\|u^{k}(t)\right\|_{V}^{2} \leq\left(\left\|u^{0}\right\|_{V}^{2}+\frac{2 L^{2}}{\nu\left(1-\rho^{*}\right)}\|\phi\|_{L^{2}(-h, 0 ; H)}^{2}\right) e^{-\theta t}+\frac{2}{\nu} L(\theta)
$$

independentemente de $k$. Isto garante que $u \in L^{\infty}(0, T ; V)$ com a estimativa desejada. Ainda podemos obter por descarte no primeiro membro em (3.8) que

$$
\begin{aligned}
M(\theta) \int_{0}^{t}\left\|A u^{k}(s)\right\|_{L^{2}}^{2} d s & \leq M(\theta) \int_{0}^{t} e^{\theta s}\left\|A u^{k}(s)\right\|_{L^{2}}^{2} d s \\
& \leq\|u(0)\|_{V}^{2}+\frac{2 L^{2}}{\nu\left(1-\rho^{*}\right)}\|\phi\|_{L^{2}(-h, 0 ; H)}^{2}+\frac{2}{\nu} e^{\theta t} L(\theta),
\end{aligned}
$$

independentemente de $k$, o que nos permite concluir que $u \in L^{2}(0, T ; D(A))$.

Para provar a unicidade da solução forte, sejam $u$ e $v$ duas soluções do problema (3.4), definimos $w(t)=u(t)-v(t)$. Então

$$
\begin{aligned}
& \frac{d}{d t} u(t)+\nu A u(t)+B\left(F\left(t, u_{t}\right), u(t)\right)=f(t)+G(u(t-\rho(t))) \\
& \frac{d}{d t} v(t)+\nu A v(t)+B\left(F\left(t, v_{t}\right), v(t)\right)=f(t)+G(v(t-\rho(t))) \\
& u(0)-v(0)=u^{0}-u^{0}=0
\end{aligned}
$$




$$
u(t)-v(t)=\phi(t)-\phi(t)=0, \quad t \in(-h, 0)
$$

e portanto, usando

$$
\begin{aligned}
B\left(F\left(t, v_{t}\right), v(t)\right) & -B\left(F\left(t, u_{t}\right), u(t)\right) \\
& =-B\left(F\left(t, u_{t}\right)-F\left(t, v_{t}\right), u(t)\right)-B\left(F\left(t, v_{t}\right), u(t)-v(t)\right) \\
& =-B\left(F\left(t, u_{t}\right)-F\left(t, v_{t}\right), u(t)\right)-B\left(F\left(t, v_{t}\right), w(t)\right),
\end{aligned}
$$

temos que $w$ satisfaz

$$
\begin{aligned}
& \frac{d}{d t} w(t)+\nu A w(t)=-B\left(F\left(t, u_{t}\right)-F\left(t, v_{t}\right), u(t)\right) \\
&-B\left(F\left(t, v_{t}\right), w(t)\right)+G(u(t-\rho(t)))-G(v(t-\rho(t))) \\
& w(0)=0 \\
& w(t)=0, \quad t \in(-h, 0)
\end{aligned}
$$

Tomando o produto escalar em $L^{2}$, com $w(t)$ temos

$$
\begin{aligned}
\frac{d}{d t}\|w(t)\|_{L^{2}}^{2}+ & 2 \nu\langle A w(t), w(t)\rangle \\
=- & 2 b\left(F\left(t, u_{t}\right)-F\left(t, v_{t}\right), u(t), w(t)\right)-2 b\left(F\left(t, v_{t}\right), w(t), w(t)\right) \\
& +2\langle G(u(t-\rho(t)))-G(v(t-\rho(t))), w(t)\rangle .
\end{aligned}
$$

Mas como

$$
\begin{aligned}
b\left(F\left(t, u_{t}\right)-F\left(t, v_{t}\right), u(t), w(t)\right) & \leq \int_{\Omega}\left|\left(F\left(t, u_{t}\right)-F\left(t, v_{t}\right)\right) \nabla u(t) w(t)\right| d x \\
& \leq\left\|F\left(t, u_{t}\right)-F\left(t, v_{t}\right)\right\|_{L^{4}}\|\nabla u\|_{L^{2}}\|w\|_{L^{4}}
\end{aligned}
$$

e como

$$
\begin{aligned}
\left\|F\left(t, u_{t}\right)-F\left(t, v_{t}\right)\right\|_{L^{4}} & \leq C\left\|F\left(t, u_{t}\right)-F\left(t, v_{t}\right)\right\|_{V} \\
\|\nabla u\|_{L^{2}} & =\|u\|_{V} \\
\|w\|_{L^{4}} & \leq C\|w\|_{V}^{\frac{3}{4}}\|w\|_{L^{2}}^{\frac{1}{4}}
\end{aligned}
$$

então

$$
\begin{aligned}
b\left(F\left(t, u_{t}\right)\right. & \left.-F\left(t, v_{t}\right), u(t), w(t)\right) \\
& \leq C\left\|F\left(t, u_{t}\right)-F\left(t, v_{t}\right)\right\|_{V}\|u(t)\|_{V}\|w(t)\|_{V}^{\frac{3}{4}}\|w(t)\|_{L^{2}}^{\frac{1}{4}} \\
& \leq \frac{\nu\left(1-\tau^{*}\right)}{2}\left\|F\left(t, u_{t}\right)-F\left(t, v_{t}\right)\right\|_{V}^{2}+\|u(t)\|_{V}^{2} \frac{2 C}{\nu\left(1-\tau^{*}\right)}\|w(t)\|_{V}^{\frac{3}{2}}\|w(t)\|_{L^{2}}^{\frac{1}{2}} .
\end{aligned}
$$

Usando agora a desigualdade de Young, com $p=\frac{4}{3}$ e $q=4$, na segunda parcela do segundo membro, temos

$$
\|u(t)\|_{V}^{2} \frac{2 C}{\nu\left(1-\tau^{*}\right)}\|w(t)\|_{V}^{\frac{3}{2}}\|w(t)\|_{L^{2}}^{\frac{1}{2}}
$$




$$
\begin{aligned}
& =\|u(t)\|_{V}^{2} \frac{2 C}{\nu\left(1-\tau^{*}\right)}\left(\frac{4}{3} \frac{\nu}{2}\right)^{\frac{3}{4}}\|w(t)\|_{V}^{\frac{3}{2}}\left(\frac{3}{4} \frac{2}{\nu}\right)^{\frac{3}{4}}\|w(t)\|_{L^{2}}^{\frac{1}{2}} \\
& \leq \frac{3}{4} \frac{\nu}{2} \frac{4}{3}\|w(t)\|_{V}^{2}+\frac{1}{4}\left(\frac{2 C}{\nu\left(1-\tau^{*}\right)}\left(\frac{3}{4} \frac{2}{\nu}\right)^{\frac{3}{4}}\right)^{4}\|u(t)\|_{V}^{8}\|w(t)\|_{L^{2}}^{2} \\
& \leq \frac{\nu}{2}\|w(t)\|_{V}^{2}+\frac{C^{4}}{4} \frac{2^{7}}{\nu^{7}\left(1-\tau^{*}\right)^{4}} \frac{3^{3}}{4^{3}}\|u\|_{L^{\infty}(0, T ; V)}^{8}\|w(t)\|_{L^{2}}^{2} \\
& \leq \frac{\nu}{2}\|w(t)\|_{V}^{2}+\frac{27 C^{4}}{2 \nu^{7}\left(1-\tau^{*}\right)^{4}}\|u\|_{L^{\infty}(0, T ; V)}^{8}\|w(t)\|_{L^{2}}^{2},
\end{aligned}
$$

donde segue que

$$
\begin{aligned}
b\left(F\left(t, u_{t}\right)-F\left(t, v_{t}\right), u(t), w(t)\right) \leq & \frac{\nu\left(1-\tau^{*}\right)}{2}\left\|F\left(t, u_{t}\right)-F\left(t, v_{t}\right)\right\|_{V}^{2}+\frac{\nu}{2}\|w(t)\|_{V}^{2} \\
& +\frac{27 C^{4}}{2 \nu^{7}\left(1-\tau^{*}\right)^{4}}\|u\|_{L^{\infty}(0, T ; V)}^{8}\|w(t)\|_{L^{2}}^{2} .
\end{aligned}
$$

Levando agora em conta que

$$
2 \nu\langle A w(t), w(t)\rangle=2 \nu\langle\nabla w(t), \nabla w(t)\rangle=2 \nu\|w(t)\|_{V}^{2},
$$

e que

$$
\begin{aligned}
2\langle G(u(t-\rho(t))) & -G(v(t-\rho(t))), w(t)\rangle \\
& \leq 2\|G(u(t-\rho(t)))-G(v(t-\rho(t)))\|_{L^{2}}\|w(t)\|_{L^{2}} \\
& \leq \frac{2}{\nu}\|G(u(t-\rho(t)))-G(v(t-\rho(t)))\|_{L^{2}}^{2}+\frac{\nu}{2}\|w(t)\|_{L^{2}}^{2},
\end{aligned}
$$

temos

$$
\begin{aligned}
\frac{d}{d t}\|w(t)\|_{L^{2}}^{2}+2 \nu\|w(t)\|_{V}^{2} \leq & \frac{\nu\left(1-\tau^{*}\right)}{2}\left\|F\left(t, u_{t}\right)-F\left(t, v_{t}\right)\right\|_{V}^{2}+\frac{\nu}{2}\|w(t)\|_{V}^{2} \\
& +\frac{27 C^{4}}{2 \nu^{7}\left(1-\tau^{*}\right)^{4}}\|u\|_{L^{\infty}(0, T ; V)}^{8}\|w(t)\|_{L^{2}}^{2} \\
& +\frac{2}{\nu}\|G(u(t-\rho(t)))-G(v(t-\rho(t)))\|_{L^{2}}^{2} \\
& +\frac{\nu}{2}\|w(t)\|_{L^{2}}^{2} .
\end{aligned}
$$

Integrando no tempo de 0 a $t$, temos

$$
\begin{aligned}
\|w(t)\|_{L^{2}}^{2}+ & 2 \nu \int_{0}^{t}\|w(s)\|_{V}^{2} d s \\
\leq & \frac{\nu\left(1-\tau^{*}\right)}{2} \int_{0}^{t}\left\|F\left(s, u_{s}\right)-F\left(s, v_{s}\right)\right\|_{V}^{2} d s \\
& +\frac{\nu}{2} \int_{0}^{t}\|w(s)\|_{V}^{2} d s+\frac{27 C^{4}}{2 \nu^{7}\left(1-\tau^{*}\right)^{4}}\|u\|_{L^{\infty}(0, T ; V)}^{8} \int_{0}^{t}\|w(s)\|_{L^{2}}^{2} d s \\
& +\frac{2}{\nu} \int_{0}^{t}\|G(u(s-\rho(s)))-G(v(s-\rho(s)))\|_{L^{2}}^{2} d s \\
& +\frac{\nu}{2} \int_{0}^{t}\|w(s)\|_{L^{2}}^{2} d s .
\end{aligned}
$$


Usando agora a mudança de variáveis $\eta=s-\tau(s)$ vemos que

$$
\begin{aligned}
\int_{0}^{t} \| F\left(s, u_{s}\right) & -F\left(s, v_{s}\right) \|_{V}^{2} d s \\
& =\int_{0}^{t}\|u(s-\tau(s))-v(s-\tau(s))\|_{V}^{2} d s \\
& \leq \frac{1}{1-\tau^{*}} \int_{-\tau(0)}^{t-\tau(t)}\|u(\eta)-v(\eta)\|_{V}^{2} d \eta \\
& \leq \frac{1}{1-\tau^{*}} \int_{-h}^{t}\|w(\eta)\|_{V}^{2} d \eta=\frac{1}{1-\tau^{*}} \int_{0}^{t}\|w(\eta)\|_{V}^{2} d \eta,
\end{aligned}
$$

e da mesma forma com $\eta=s-\rho(s)$, e a hipótese de Lipschitz de $G$, temos

$$
\begin{aligned}
\int_{0}^{t} \| G(u(s-\rho(s))) & -G(v(s-\rho(s))) \|_{L^{2}}^{2} d s \\
& \leq L^{2} \int_{0}^{t}\|u(s-\rho(s))-v(s-\rho(s))\|_{L^{2}}^{2} d s \\
& \leq \frac{L^{2}}{1-\rho^{*}} \int_{-\rho(0)}^{t-\rho(t)}\|u(\eta)-v(\eta)\|_{L^{2}}^{2} d \eta \\
& \leq \frac{L^{2}}{1-\rho^{*}} \int_{-h}^{t}\|w(\eta)\|_{L^{2}}^{2} d \eta=\frac{L^{2}}{1-\rho^{*}} \int_{0}^{t}\|w(\eta)\|_{L^{2}}^{2} d \eta .
\end{aligned}
$$

Usando estas duas limitações temos

$$
\begin{aligned}
\|w(t)\|_{L^{2}}^{2}+ & 2 \nu \int_{0}^{t}\|w(s)\|_{V}^{2} d s \\
\leq & \frac{\nu\left(1-\tau^{*}\right)}{2} \frac{1}{\left(1-\tau^{*}\right)} \int_{0}^{t}\|w(s)\|_{V}^{2} d s \\
& +\frac{\nu}{2} \int_{0}^{t}\|w(s)\|_{V}^{2} d s+\frac{27 C^{4}}{2 \nu^{7}\left(1-\tau^{*}\right)^{4}}\|u\|_{L^{\infty}(0, T ; V)}^{8} \int_{0}^{t}\|w(s)\|_{L^{2}}^{2} d s \\
& +\frac{2 L^{2}}{\nu\left(1-\rho^{*}\right)} \int_{0}^{t}\|w(s)\|_{L^{2}}^{2} d s+\frac{\nu}{2} \int_{0}^{t}\|w(s)\|_{L^{2}}^{2} d s .
\end{aligned}
$$

Finalmente

$$
\begin{aligned}
\|w(t)\|_{L^{2}}^{2} & +\nu \int_{0}^{t}\|w(s)\|_{V}^{2} d s \\
& \leq\left(\frac{\nu}{2}+\frac{2 L^{2}}{\nu\left(1-\rho^{*}\right)}+\frac{27 C^{4}}{2 \nu^{7}\left(1-\tau^{*}\right)^{4}}\|u\|_{L^{\infty}(0, T ; V)}^{8}\right) \int_{0}^{t}\|w(s)\|_{L^{2}}^{2} d s .
\end{aligned}
$$

A unicidade segue agora do lema de Gronwall, após descarte da integral do primeiro membro. Isto completa esta prova.

Nota 3.2.3. Em particular, se $f=0$, então qualquer solução forte do problema (3.4) decai a zero exponencialmente.

Novamente, já que nas condições impostas temos existência e unicidade de solução para o problema (3.4) podemos determinar a pressão $p(t, x)$, usando a equação (3.1). Da mesma 
forma que no capítulo anterior, a técnica usada em Temam [26] permite determinar a pressão que é única a menos de uma constante.

Agora, como consequência do Teorema 3.2.2, provaremos a existência de conjuntos absorventes nos espaço $C([-h, 0] ; V)$. Lembremos a definição de conjunto absorvente para o problema (3.4).

Definição 3.2.4. Seja $u=u\left(t ; u^{0}, \phi\right)$ a solução forte do problema (3.4). Seja $B_{V}(0, \alpha) a$ bola de $C([-h, 0] ; V)$ com raio $\alpha$ e centro 0 . Seja $\phi \in C([-h, 0] ; V)$ tal que $\phi(0)=u^{0} e$ $\|\phi\|_{C([-h, 0] ; V)}<R$, para algum $R>0$ fixo. Se existe um tempo $T_{0}=T_{0}(R)>0$ tal que $\left\|u\left(t ; u^{0}, \phi\right)\right\|_{V}<\alpha$ para todo $t \geq T_{0}$, então a bola $B_{V}(0, \alpha)$ é dita um conjunto absorvente em $C([-h, 0] ; V)$.

Corolário 3.2.5. Suponha que as condições do Teorema 3.2 .2 são satisfeitas. Então existe um conjunto absorvente em $C([-h, 0] ; V)$ para o problema (3.4).

Prova. Seja $R>0$ e $\phi \in B_{V}(0, R)$. Note que

$$
\left\|u^{0}\right\|_{V}=\|\phi(0)\|_{V} \leq \max _{-h \leq t \leq 0}\|\phi(t)\|_{V}=\|\phi\|_{C([-h, 0] ; V)} \leq R .
$$

Do teorema 3.2.2, temos que

$$
\begin{aligned}
\|u(t)\|_{V}^{2} & \leq e^{-\theta t}\left(\left\|u^{0}\right\|_{V}^{2}+\frac{2 L^{2}}{\nu\left(1-\rho^{*}\right)}\|\phi\|_{L^{2}(-h, 0 ; H)}^{2}\right)+\frac{2}{\nu} L(\theta) . \\
& \leq e^{-\theta t}\left(R^{2}+\frac{2 L^{2}}{\nu\left(1-\rho^{*}\right)} \int_{-h}^{0}\|\phi(s)\|_{H}^{2} d s\right)+\frac{2}{\nu} L(\theta) . \\
& \leq e^{-\theta t}\left(R^{2}+\frac{2 L^{2}}{\nu \lambda_{1}\left(1-\rho^{*}\right)} \int_{-h}^{0}\|\phi(s)\|_{V}^{2} d s\right)+\frac{2}{\nu} L(\theta) . \\
& \leq e^{-\theta t}\left(R^{2}+\frac{2 L^{2}}{\nu \lambda_{1}\left(1-\rho^{*}\right)}\|\phi\|_{C([-h, 0] ; V)}^{2} \int_{-h}^{0} 1 d s\right)+\frac{2}{\nu} L(\theta) . \\
& \leq e^{-\theta t}\left(R^{2}+\frac{2 L^{2}}{\nu \lambda_{1}\left(1-\rho^{*}\right)} h R^{2}\right)+\frac{2}{\nu} L(\theta) .
\end{aligned}
$$

Afirmamos que desta desigualdade as bolas $B_{V}(0, \alpha) \operatorname{com} \alpha^{2}>\frac{2}{\nu} L(\theta)$ são conjuntos absorventes em $C([-h, 0] ; V)$, já que

$$
\|u(t)\|_{V} \leq \alpha \quad \text { para todo } \quad t \geq T_{0}=T_{0}(R)=\frac{1}{\theta} \ln \frac{R^{2}+\frac{2 L^{2}}{\nu \lambda_{1}\left(1-\rho^{*}\right)} h R^{2}}{\alpha^{2}-\frac{2}{\nu} L(\theta)} .
$$

De fato, para $t \geq T_{0}(R)$, temos

$$
\begin{aligned}
\|u(t)\|_{V}^{2} & \leq e^{-\theta\left(\frac{1}{\theta} \ln \frac{R^{2}+\frac{2 L^{2}}{\nu \lambda_{1}\left(1-\rho^{*}\right)} h R^{2}}{\alpha^{2}-\frac{2}{\nu} L(\theta)}\right)}\left(R^{2}+\frac{2 L^{2}}{\nu \lambda_{1}\left(1-\rho^{*}\right)} h R^{2}\right)+\frac{2}{\nu} L(\theta) \\
& =\frac{\alpha^{2}-\frac{2}{\nu} L(\theta)}{R^{2}+\frac{2 L^{2}}{\nu \lambda_{1}\left(1-\rho^{*}\right)} h R^{2}}\left(R^{2}+\frac{2 L^{2}}{\nu \lambda_{1}\left(1-\rho^{*}\right)} h R^{2}\right)+\frac{2}{\nu} L(\theta) \\
& =\alpha^{2}-\frac{2}{\nu} L(\theta)+\frac{2}{\nu} L(\theta)=\alpha^{2} .
\end{aligned}
$$

Esta prova está completa. 


\subsection{Estimativa de energia para a solução fraca}

Nesta seção iremos obter uma estimativa de energia para a solução fraca do Teorema 3.2.1. O procedimento adotado será o mesmo de Temam [27]. Multiplicando cada equação em (3.5) pela respectiva $\gamma_{j k}$ e somando em $j$, obtemos

$$
\frac{1}{2} \frac{d}{d t}\left\|u^{k}(t)\right\|_{L^{2}}^{2}+\nu\left\|u^{k}(t)\right\|_{V}^{2}=\left\langle f(t), u^{k}(t)\right\rangle+\left\langle G\left(u^{k}(t-\rho(t))\right), u^{k}(t)\right\rangle .
$$

Multiplicando agora esta igualdade por $\phi(t) \in \mathcal{D}((0, T))$ com $\phi(t) \geq 0$, e integrando em $t$ de 0 a $T$, obtemos

$$
\begin{aligned}
\int_{0}^{T}\left(\frac{1}{2} \frac{d}{d t}\right. & \left.\left\|u^{k}(t)\right\|_{L^{2}}^{2}+\nu\left\|u^{k}(t)\right\|_{V}^{2}\right) \phi(t) d t \\
& =\int_{0}^{T}\left(\left\langle f(t), u^{k}(t)\right\rangle+\left\langle G\left(u^{k}(t-\rho(t))\right), u^{k}(t)\right\rangle\right) \phi(t) d t .
\end{aligned}
$$

Mas note que

$$
\int_{0}^{T} \frac{1}{2} \frac{d}{d t}\left\|u^{k}(t)\right\|_{L^{2}}^{2} \phi(t) d t=-\frac{1}{2} \int_{0}^{T}\left\|u^{k}(t)\right\|_{L^{2}} \frac{d}{d t} \phi(t) d t
$$

donde temos

$$
\begin{aligned}
\frac{1}{2} \int_{0}^{T}-\left\|u^{k}(t)\right\|_{L^{2}}^{2} & \frac{d}{d t} \phi(t)+\nu\left\|u^{k}(t)\right\|_{V}^{2} \phi(t) d t \\
& =\int_{0}^{T}\left(\left\langle f(t), u^{k}(t)\right\rangle+\left\langle G\left(u^{k}(t-\rho(t))\right), u^{k}(t)\right\rangle\right) \phi(t) d t .
\end{aligned}
$$

Passando agora o limite inferior, quando $k \rightarrow \infty$, chegamos a

$$
\begin{aligned}
\frac{1}{2} \int_{0}^{T}-\|u(t)\|_{L^{2}}^{2} & \frac{d}{d t} \phi(t)+\nu\|u(t)\|_{V}^{2} \phi(t) d t \\
& \leq \int_{0}^{T}(\langle f(t), u(t)\rangle+\langle G(u(t-\rho(t))), u(t)\rangle) \phi(t) d t,
\end{aligned}
$$

ou ainda

$$
\begin{aligned}
\frac{1}{2} \int_{0}^{T} \frac{d}{d t}\|u(t)\|_{L^{2}}^{2} \phi(t)+\nu\|u(t)\|_{V}^{2} \phi(t) d t \\
\quad \leq \int_{0}^{T}(\langle f(t), u(t)\rangle+\langle G(u(t-\rho(t))), u(t)\rangle) \phi(t) d t
\end{aligned}
$$

para toda $\phi \in \mathcal{D}((0, T)), \phi \geq 0$. Isto significa que

$$
\frac{1}{2} \frac{d}{d t}\|u(t)\|_{L^{2}}^{2}+\nu\|u(t)\|_{V}^{2} \leq\langle f(t), u(t)\rangle+\langle G(u(t-\rho(t))), u(t)\rangle .
$$

para quase todo $t \in[0, T]$. Reescrevendo, obtemos

$$
\left\langle\frac{d}{d t} u(t), u(t)\right\rangle_{L^{2}}+\nu\|u(t)\|_{V}^{2} \leq\langle f(t), u(t)\rangle+\langle G(u(t-\rho(t))), u(t)\rangle
$$

para quase todo $t \in[0, T]$. 


\subsection{Estabilidade de soluções estacionárias}

Nesta seção vamos considerar que a força externa $f$ não depende de $t$. A equação estacionária associada ao problema (3.4) é

$$
\nu A u+B(u, u)=f+G(u) .
$$

Vamos provar que esta equação possui solução estacionária $u_{\infty}$. A demonstração está feita em [3], mas por motivos de completamento, iremos aqui escrevê-la.

Teorema 3.4.1. Suponha $f \in V^{\prime}$ e $\nu>\frac{L}{\lambda_{1}}$ onde $\lambda_{1}$ é o primeiro autovalor do operador A. Então, o problema estacionário

$$
\nu A u+B(u, u)=f+G(u)
$$

possui solução estacionária $u_{\infty} \in V$. Se além disso $\left(\nu-\frac{L}{\lambda_{1}}\right)^{2}>C_{1}^{2}\|f\|_{V^{\prime}}$, onde $C_{1}$ é a constante de inclusão de $V$ em $L^{4}$, então, a solução é única. Se $f \in H$ então $u_{\infty} \in D(A)$.

Prova. $V$ é um espaço de Hilbert. Para cada $z \in V$, temos que

$$
\nu\langle\cdot, \cdot\rangle_{V}+b(z, \cdot, \cdot): V \times V \rightarrow \mathbb{R}
$$

é bilinear, contínua e coerciva. E como

$$
f+\langle G(z), \cdot\rangle_{L^{2}} \in V^{\prime}
$$

então pelo teorema de Lax-Milgram, existe uma única $u \in V$ tal que

$$
\nu\langle u, v\rangle_{V}+b(z, u, v)=\langle f, v\rangle+\langle G(z), v\rangle
$$

para toda $v \in V$. A unicidade de $u$ permite definir a aplicação $\varphi$ que a cada $z \in V$ associa $u=\varphi(z)$, tal que (3.11) é satisfeita. Tomando $v=u$ em (3.11) temos

$$
\nu\|u\|_{V}^{2}=\langle f, u\rangle+\langle G(z), u\rangle
$$

donde

$$
\begin{aligned}
\nu\|u\|_{V}^{2} & \leq\|f\|_{V^{\prime}}\|u\|_{V}+\|G(z)\|_{L^{2}}\|u\|_{L^{2}} \\
& \leq\|f\|_{V^{\prime}}\|u\|_{V}+L\|z\|_{L^{2}}\|u\|_{L^{2}} \\
& \leq\|f\|_{V^{\prime}}\|u\|_{V}+\frac{L}{\lambda_{1}}\|z\|_{V}\|u\|_{V}
\end{aligned}
$$

e portanto

$$
\nu\|u\|_{V} \leq\|f\|_{V^{\prime}}+\frac{L}{\lambda_{1}}\|z\|_{V} .
$$


Tomemos $k>0$ tal que $k\left(\nu-\frac{L}{\lambda_{1}}\right)>\|f\|_{V^{\prime}}$, e consideremos o conjunto

$$
X=\left\{z \in V ; \quad\|z\|_{V} \leq k\right\} .
$$

O conjunto $X$ é um conjunto convexo e compacto de $\left(L^{4}(\Omega)\right)^{3}$, em virtude da imersão compacta de $V$ em $\left(L^{4}(\Omega)\right)^{3}$. Mais ainda, por (3.12) e da escolha de $k$ temos que a aplicação $\varphi$ leva $X$ em $X$.

Para provarmos a continuidade desta aplicação $\varphi$, sejam $z_{1}, z_{2} \in X$ e $u_{1}, u_{2} \in X$ tais que

$$
\nu\left\langle u_{i}, v\right\rangle_{V}+b\left(z_{i}, u_{i}, v\right)=\langle f, v\rangle+\left\langle G\left(z_{i}\right), v\right\rangle \quad(i=1,2)
$$

para toda $v \in V$. Fazendo a diferença entre estas duas igualdades, segue que

$$
\nu\left\langle u_{1}-u_{2}, v\right\rangle_{V}=b\left(z_{2}, u_{2}, v\right)-b\left(z_{1}, u_{1}, v\right)+\left\langle G\left(z_{1}\right)-G\left(z_{2}\right), v\right\rangle
$$

ou ainda

$$
\nu\left\langle u_{1}-u_{2}, v\right\rangle_{V}=b\left(z_{2}-z_{1}, u_{1}, v\right)-b\left(z_{2}, u_{1}-u_{2}, v\right)+\left\langle G\left(z_{1}\right)-G\left(z_{2}\right), v\right\rangle
$$

e escolhendo $v=u_{1}-u_{2}$, obtemos

$$
\begin{aligned}
\nu\left\|u_{1}-u_{2}\right\|_{V}^{2} & =b\left(z_{2}-z_{1}, u_{1}, u_{1}-u_{2}\right)+\left\langle G\left(z_{1}\right)-G\left(z_{2}\right), u_{1}-u_{2}\right\rangle \\
& \leq\left\|z_{1}-z_{2}\right\|_{L^{4}}\left\|\nabla u_{1}\right\|_{L^{2}}\left\|u_{1}-u_{2}\right\|_{L^{4}}+\left\|G\left(z_{1}\right)-G\left(z_{2}\right)\right\|_{L^{2}}\left\|u_{1}-u_{2}\right\|_{L^{2}} \\
& \leq C_{1}\left\|z_{1}-z_{2}\right\|_{L^{4}}\left\|u_{1}\right\|_{V}\left\|u_{1}-u_{2}\right\|_{V}+\frac{L}{\sqrt{\lambda_{1}}}\left\|z_{1}-z_{2}\right\|_{L^{2}}\left\|u_{1}-u_{2}\right\|_{V} \\
& \leq k C_{1}\left\|z_{1}-z_{2}\right\|_{L^{4}}\left\|u_{1}-u_{2}\right\|_{V}+\frac{L}{\sqrt{\lambda_{1}}}\left\|z_{1}-z_{2}\right\|_{L^{2}}\left\|u_{1}-u_{2}\right\|_{V},
\end{aligned}
$$

onde $C_{1}$ é a constante de imersão de $V$ em $\left(L^{4}(\Omega)\right)^{3}$. Obtemos assim,

$$
\nu\left\|u_{1}-u_{2}\right\|_{V} \leq k C_{1}\left\|z_{1}-z_{2}\right\|_{L^{4}}+\frac{L}{\sqrt{\lambda_{1}}}\left\|z_{1}-z_{2}\right\|_{L^{2}} .
$$

Como consequência desta última desigualdade e das inclusões $V \hookrightarrow\left(L^{4}(\Omega)\right)^{3} \hookrightarrow\left(L^{2}(\Omega)\right)^{3}$, temos

$$
\begin{aligned}
\frac{\nu}{C_{1}}\left\|u_{1}-u_{2}\right\|_{L^{4}} & \leq \frac{\nu C_{1}}{C_{1}}\left\|u_{1}-u_{2}\right\|_{V} \\
& \leq k C_{1}\left\|z_{1}-z_{2}\right\|_{L^{4}}+\frac{L}{\sqrt{\lambda_{1}}}\left\|z_{1}-z_{2}\right\|_{L^{2}} \\
& \leq k C_{1}\left\|z_{1}-z_{2}\right\|_{L^{4}}+\frac{L C_{2}}{\sqrt{\lambda_{1}}}\left\|z_{1}-z_{2}\right\|_{L^{4}} \\
& =\left(k C_{1}+\frac{L C_{2}}{\sqrt{\lambda_{1}}}\right)\left\|z_{1}-z_{2}\right\|_{L^{4}},
\end{aligned}
$$

onde $C_{2}$ é a constante de inclusão de $\left(L^{4}(\Omega)\right)^{3}$ em $\left(L^{2}(\Omega)\right)^{3}$. Segue que $\varphi$ é contínua de $L^{4}$ em $L^{4}$. O teorema de ponto fixo de Schauder garante agora que existe um ponto fixo para $\varphi$ e tal ponto fixo é solução estacionária de (3.10). 
Para provar a unicidade sejam $u_{1}$ e $u_{2}$ duas soluções estacionárias de (3.10). Então

$$
\nu A u_{1}+B\left(u_{1}, u_{1}\right)=f+G\left(u_{1}\right)
$$

e também

$$
\nu A u_{2}+B\left(u_{2}, u_{2}\right)=f+G\left(u_{2}\right) .
$$

Fazendo a diferença entre estas equações, e colocando $w=u_{1}-u_{2}$, temos

$$
\nu A w+B\left(u_{1}, u_{1}\right)-B\left(u_{2}, u_{2}\right)=G\left(u_{1}\right)-G\left(u_{2}\right) .
$$

Aplicando esta equação em $w$, obtemos

$$
\nu\langle A w, w\rangle+b\left(u_{1}, u_{1}, w\right)-b\left(u_{2}, u_{2}, w\right)=\left\langle G\left(u_{1}\right)-G\left(u_{2}\right), w\right\rangle .
$$

Como

$$
\nu\langle A w, w\rangle=\nu\langle\nabla w, \nabla w\rangle=\nu\|\nabla w\|_{L^{2}}^{2}=\nu\|w\|_{V}^{2},
$$

e

$$
\begin{aligned}
b\left(u_{1}, u_{1}, w\right) & -b\left(u_{2}, u_{2}, w\right) \\
& =b\left(u_{1}, w, w\right)+b\left(u_{1}, u_{2}, w\right)-b\left(u_{2}, u_{2}, w\right) \\
& =b\left(u_{1}, w, w\right)-b\left(w, u_{2}, w\right) \\
& =-b\left(w, u_{2}, w\right),
\end{aligned}
$$

então

$$
\begin{aligned}
\nu\|w\|_{V}^{2} & \leq\left|b\left(w, u_{2}, w\right)\right|+\left\|G\left(u_{1}\right)-G\left(u_{2}\right)\right\|_{L^{2}}\|w\|_{L^{2}} \\
& \leq\|w\|_{L^{4}}\left\|\nabla u_{2}\right\|_{L^{2}}\|w\|_{L^{4}}+\frac{L}{\sqrt{\lambda_{1}}}\left\|u_{1}-u_{2}\right\|_{L^{2}}\|w\|_{V} \\
& \leq C_{1}^{2}\|w\|_{V}\left\|u_{2}\right\|_{V}\|w\|_{V}+\frac{L}{\lambda_{1}}\left\|u_{1}-u_{2}\right\|_{V}\|w\|_{V} \\
& =C_{1}^{2}\left\|u_{2}\right\|_{V}\|w\|_{V}^{2}+\frac{L}{\lambda_{1}}\|w\|_{V}^{2}
\end{aligned}
$$

Mas, aplicando (3.13) em $u_{2}$, obtemos

$$
\begin{aligned}
\nu\left\|u_{2}\right\|_{V}^{2} & =\left\langle f, u_{2}\right\rangle+\left\langle G\left(u_{2}\right), u_{2}\right\rangle \\
& \leq\|f\|_{V^{\prime}}\left\|u_{2}\right\|_{V}+\left\|G\left(u_{2}\right)\right\|_{L^{2}}\left\|u_{2}\right\|_{L^{2}} \\
& \leq\|f\|_{V^{\prime}}\left\|u_{2}\right\|_{V}+\frac{L}{\lambda_{1}}\left\|u_{2}\right\|_{V}^{2},
\end{aligned}
$$

e portanto

$$
\left\|u_{2}\right\|_{V} \leq \frac{\|f\|_{V^{\prime}}}{\left(\nu-\frac{L}{\lambda_{1}}\right)}
$$


Desta desigualdade, temos

$$
\nu\|w\|_{V}^{2} \leq \frac{C_{1}^{2}\|f\|_{V^{\prime}}}{\left(\nu-\frac{L}{\lambda_{1}}\right)}\|w\|_{V}^{2}+\frac{L}{\lambda_{1}}\|w\|_{V}^{2}
$$

Reorganizando os termos, segue que

$$
\left(\left(\nu-\frac{L}{\lambda_{1}}\right)^{2}-C_{1}^{2}\|f\|_{V^{\prime}}\right)\|w\|_{V}^{2} \leq 0
$$

e pela condição assumida, temos a unicidade da solução.

Se $f \in H$ então, denotando $u_{\infty}$ a solução da equação estacionária, temos

$$
\nu A u_{\infty}+B\left(u_{\infty}, u_{\infty}\right)=f+G\left(u_{\infty}\right)
$$

e tomando o produto interno em $L^{2} \operatorname{com} u_{\infty}$, podemos estimar $\left\|u_{\infty}\right\|_{V}$ da seguinte forma,

$$
\begin{aligned}
\nu\left\|u_{\infty}\right\|_{V}^{2} & \leq\|f\|_{L^{2}}\left\|u_{\infty}\right\|_{L^{2}}+\left\|G\left(u_{\infty}\right)\right\|_{L^{2}}\left\|u_{\infty}\right\|_{L^{2}} \\
& \leq \frac{1}{\sqrt{\lambda_{1}}}\|f\|_{L^{2}}\left\|u_{\infty}\right\|_{V}+L\left\|u_{\infty}\right\|_{L^{2}}\left\|u_{\infty}\right\|_{L^{2}} \\
& \leq \frac{1}{\sqrt{\lambda_{1}}}\|f\|_{L^{2}}\left\|u_{\infty}\right\|_{V}+\frac{L}{\lambda_{1}}\left\|u_{\infty}\right\|_{V}\left\|u_{\infty}\right\|_{V},
\end{aligned}
$$

ou ainda,

$$
\left\|u_{\infty}\right\|_{V} \leq \frac{1}{\sqrt{\lambda_{1}}\left(\nu-\frac{L}{\lambda_{1}}\right)}\|f\|_{L^{2}}
$$

Além disso, usando a desigualdade (3.2) podemos obter,

$$
\left\|B\left(u_{\infty}, u_{\infty}\right)\right\|_{L^{2}} \leq C_{3}\left\|u_{\infty}\right\|_{V}^{\frac{3}{2}}\left\|A u_{\infty}\right\|_{L^{2}}^{\frac{1}{2}} .
$$

Desta forma, temos a estimativa para $\left\|A u_{\infty}\right\|_{L^{2}}$ dada por

$$
\begin{aligned}
\nu\left\|A u_{\infty}\right\|_{L^{2}} & \leq\|f\|_{L^{2}}+\left\|B\left(u_{\infty}, u_{\infty}\right)\right\|_{L^{2}}+\left\|G\left(u_{\infty}\right)\right\|_{L^{2}} \\
& \leq\|f\|_{L^{2}}+C_{3}\left\|u_{\infty}\right\|_{V}^{\frac{3}{2}}\left\|A u_{\infty}\right\|_{L^{2}}^{\frac{1}{2}}+L\left\|u_{\infty}\right\|_{L^{2}} \\
& \leq\|f\|_{L^{2}}+\frac{C_{3}^{2}}{2 \nu}\left\|u_{\infty}\right\|_{V}^{3}+\frac{\nu}{2}\left\|A u_{\infty}\right\|_{L^{2}}+\frac{L}{\sqrt{\lambda_{1}}}\left\|u_{\infty}\right\|_{V} \\
& \leq\|f\|_{L^{2}}+\frac{C_{3}^{2}}{2 \nu}\left\|u_{\infty}\right\|_{V}^{3}+\frac{\nu}{2}\left\|A u_{\infty}\right\|_{L^{2}}+\frac{L}{\sqrt{\lambda_{1}}}\left\|u_{\infty}\right\|_{V}
\end{aligned}
$$

donde segue a desigualdade

$$
\frac{\nu}{2}\left\|A u_{\infty}\right\|_{L^{2}} \leq\|f\|_{L^{2}}+\frac{C_{3}^{2}}{2 \nu \lambda_{1}^{\frac{3}{2}}\left(\nu-\frac{L}{\lambda_{1}}\right)^{3}}\|f\|_{L^{2}}^{3}+\frac{L}{\lambda_{1}\left(\nu-\frac{L}{\lambda_{1}}\right)}\|f\|_{L^{2}}
$$

e portanto $u_{\infty} \in D(A)$. Isto termina esta prova. 
Teorema 3.4.2. Sejam $u_{0} \in H, \phi \in L^{2}(-h, 0 ; V), f(t) \equiv f \in V^{\prime}$ e suponha $\nu>\frac{L}{\lambda_{1}} e$ também

$$
2 \nu-\frac{L}{\lambda_{1}}-\frac{C_{1}^{2}\|f\|_{V^{\prime}}^{2}}{\left(\nu-\frac{L}{\lambda_{1}}\right)^{2}}>\frac{L}{\left(1-\rho^{*}\right)}+\frac{C_{1}^{2}}{\left(1-\tau^{*}\right)}
$$

onde $C_{1}$ é a constante de inclusão de $V$ em $\left(L^{4}(\Omega)\right)^{3}$. Então qualquer solução fraca do problema (3.4), que satisfaz a estimativa de energia (3.9), converge exponencialmente, quando $t \rightarrow \infty$, para a solução estacionária $u_{\infty}$ do problema (3.10). Mais precisamente, existem constantes positivas $C$ e $\lambda$ tais que

$$
\left\|u(t)-u_{\infty}\right\|_{L^{2}}^{2} \leq C e^{-\lambda t}\left(\left\|u_{0}-u_{\infty}\right\|_{L^{2}}^{2}+\left\|\phi-u_{\infty}\right\|_{L^{2}(-h, 0 ; V)}^{2}\right) .
$$

para todo $t \geq 0$.

Prova. Do produto interno de (3.4) com $u_{\infty}$ em $\left(L^{2}(\Omega)\right)^{3}$, obtemos

$$
\begin{aligned}
\left\langle\frac{d}{d t} u(t), u_{\infty}\right\rangle+\nu\left\langle\nabla u(t), \nabla u_{\infty}\right\rangle & +b\left(u(t-\tau(t)), u(t), u_{\infty}\right) \\
& =\left\langle f, u_{\infty}\right\rangle+\left\langle G(u(t-\rho(t))), u_{\infty}\right\rangle
\end{aligned}
$$

ou ainda,

$$
\begin{aligned}
\left\langle\frac{d}{d t} u(t), u_{\infty}\right\rangle & +\nu\left\langle u(t), u_{\infty}\right\rangle_{V}+b\left(u(t-\tau(t)), u(t), u_{\infty}\right) \\
& =\left\langle f, u_{\infty}\right\rangle+\left\langle G(u(t-\rho(t))), u_{\infty}\right\rangle .
\end{aligned}
$$

Lembremos que $u_{\infty} \in V$ satisfaz

$$
\nu A u_{\infty}+B\left(u_{\infty}, u_{\infty}\right)=f+G\left(u_{\infty}\right)
$$

Do produto interno de (3.16) com $u(t)$ em $\left(L^{2}(\Omega)\right)^{3}$, obtemos

$$
\nu\left\langle\nabla u_{\infty}, \nabla u(t)\right\rangle+b\left(u_{\infty}, u_{\infty}, u(t)\right)=\langle f, u(t)\rangle+\left\langle G\left(u_{\infty}\right), u(t)\right\rangle
$$

ou ainda,

$$
\nu\left\langle u_{\infty}, u(t)\right\rangle_{V}+b\left(u_{\infty}, u_{\infty}, u(t)\right)=\langle f, u(t)\rangle+\left\langle G\left(u_{\infty}\right), u(t)\right\rangle .
$$

Finalmente, do produto interno de (3.16) com $u_{\infty}$, em $\left(L^{2}(\Omega)\right)^{3}$, temos

$$
\nu\left\langle\nabla u_{\infty}, \nabla u_{\infty}\right\rangle=\left\langle f, u_{\infty}\right\rangle+\left\langle G\left(u_{\infty}\right), u_{\infty}\right\rangle,
$$

donde chegamos a

$$
\nu\left\|u_{\infty}\right\|_{V}^{2}=\left\langle f, u_{\infty}\right\rangle+\left\langle G\left(u_{\infty}\right), u_{\infty}\right\rangle .
$$

Denotemos agora $w(t)=u(t)-u_{\infty}$. Somando a estimativa de energia (3.9) (para $f(t) \equiv f)$ com (3.18) e subtraindo (3.15) e (3.17), obtemos 


$$
\begin{aligned}
\left\langle\frac{d}{d t} u(t), u(t)\right\rangle_{L^{2}}+\nu\|u(t)\|_{V}^{2} & +\nu\left\|u_{\infty}\right\|_{V}^{2}-\left\langle\frac{d}{d t} u(t), u_{\infty}\right\rangle \\
& -\nu\left\langle u(t), u_{\infty}\right\rangle_{V}-b\left(u(t-\tau(t)), u(t), u_{\infty}\right) \\
& -\nu\left\langle u_{\infty}, u(t)\right\rangle_{V}-b\left(u_{\infty}, u_{\infty}, u(t)\right) \\
\leq\langle f, u(t)\rangle & +\langle G(u(t-\rho(t))), u(t)\rangle \\
& +\left\langle f, u_{\infty}\right\rangle+\left\langle G\left(u_{\infty}\right), u_{\infty}\right\rangle \\
& -\left\langle f, u_{\infty}\right\rangle-\left\langle G(u(t-\rho(t))), u_{\infty}\right\rangle \\
& -\langle f, u(t)\rangle-\left\langle G\left(u_{\infty}\right), u(t)\right\rangle .
\end{aligned}
$$

Após o cancelamento de termos, o segundo membro da equação acima pode ser reescrito na forma

$$
\left\langle G(u(t-\rho(t)))-G\left(u_{\infty}\right), w(t)\right\rangle,
$$

enquanto o primeiro membro pode ser reescrito na forma

$$
\begin{aligned}
\left\langle\frac{d}{d t} u(t), u(t)-u_{\infty}\right\rangle & +\nu\left(\|u(s)\|_{V}^{2}-\left\langle u(s), u_{\infty}\right\rangle_{V}-\left\langle u_{\infty}, u(s)\right\rangle_{V}+\left\|u_{\infty}\right\|_{V}^{2}\right) \\
& -b\left(u(t-\tau(t)), u(t), u_{\infty}\right)-b\left(u_{\infty}, u_{\infty}, u(t)\right) .
\end{aligned}
$$

Mas como

$$
-b\left(u_{\infty}, u_{\infty}, u(t)\right)=b\left(u_{\infty}, u(t), u_{\infty}\right),
$$

então

$$
\begin{aligned}
-b\left(u(t-\tau(t)), u(t), u_{\infty}\right) & -b\left(u_{\infty}, u_{\infty}, u(t)\right) \\
& =-b\left(u(t-\tau(t)), u(t), u_{\infty}\right)+b\left(u_{\infty}, u(t), u_{\infty}\right) \\
& =-b\left(w(t-\tau(t)), u(t), u_{\infty}\right) \\
& =-b\left(w(t-\tau(t)), u(t), u_{\infty}\right)+b\left(w(t-\tau(t)), u_{\infty}, u_{\infty}\right) \\
& =-b\left(w(t-\tau(t)), w(t), u_{\infty}\right) .
\end{aligned}
$$

Além disso,

$$
\begin{aligned}
\nu\left(\|u(s)\|_{V}^{2}\right. & \left.-\left\langle u(s), u_{\infty}\right\rangle_{V}-\left\langle u_{\infty}, u(s)\right\rangle_{V}+\left\|u_{\infty}\right\|_{V}^{2}\right) \\
& =\nu\left(\langle u(s), u(s)\rangle_{V}-\left\langle u_{\infty}, u(s)\right\rangle_{V}+\left\langle u_{\infty}, u_{\infty}\right\rangle_{V}-\left\langle u(s), u_{\infty}\right\rangle_{V}\right) \\
& =\nu\left(\langle w(s), u(s)\rangle_{V}-\left\langle w(s), u_{\infty}\right\rangle_{V}\right) \\
& =\nu\langle w(s), w(s)\rangle_{V} \\
& =\nu\|w(s)\|_{V}^{2} .
\end{aligned}
$$

Lembremos também que, já que $\frac{d}{d t} u_{\infty}=0$, então

$$
\left\langle\frac{d}{d t} u(t), u(t)-u_{\infty}\right\rangle=\left\langle\frac{d}{d t} w(t), w(t)\right\rangle=\frac{1}{2} \frac{d}{d t}\|w(t)\|_{L^{2}}^{2} .
$$


Desta forma, ainda podemos escrever (3.19), usando $w(t)=u(t)-u_{\infty}$, como

$$
\begin{aligned}
\frac{1}{2} \frac{d}{d t}\|w(t)\|_{L^{2}}^{2} & +\nu\|w(s)\|_{V}^{2}-b\left(w(t-\tau(t)), w(t), u_{\infty}\right) \\
& \leq\left\langle G(u(t-\rho(t)))-G\left(u_{\infty}\right), w(t)\right\rangle
\end{aligned}
$$

ou ainda,

$$
\begin{array}{r}
\frac{d}{d t}\|w(t)\|_{L^{2}}^{2} \leq-2 \nu\|w(s)\|_{V}^{2}+2 b\left(w(t-\tau(t)), w(t), u_{\infty}\right) \\
+2\left\langle G(u(t-\rho(t)))-G\left(u_{\infty}\right), w(t)\right\rangle .
\end{array}
$$

Usando esta estimativa, temos que

$$
\begin{aligned}
\frac{d}{d t}\left(e^{\lambda t}\|w(t)\|_{L^{2}}^{2}\right) & =\lambda e^{\lambda t}\|w(t)\|_{L^{2}}^{2}+e^{\lambda t} \frac{d}{d t}\|w(t)\|_{L^{2}}^{2} \\
\leq & \lambda e^{\lambda t}\|w(t)\|_{L^{2}}^{2}-2 e^{\lambda t} \nu\|w(s)\|_{V}^{2}+2 e^{\lambda t} b\left(w(t-\tau(t)), w(t), u_{\infty}\right) \\
& +2 e^{\lambda t}\left\langle G(u(t-\rho(t)))-G\left(u_{\infty}\right), w(t)\right\rangle \\
\leq & \frac{\lambda}{\lambda_{1}} e^{\lambda t}\|w(t)\|_{V}^{2}-2 e^{\lambda t} \nu\|w(t)\|_{V}^{2}+2 e^{\lambda t} b\left(w(t-\tau(t)), w(t), u_{\infty}\right) \\
& +2 e^{\lambda t}\left\|G(u(t-\rho(t)))-G\left(u_{\infty}\right)\right\|_{L^{2}}\|w(t)\|_{L^{2}} .
\end{aligned}
$$

Agora,

$$
\begin{aligned}
2 e^{\lambda t} \| G(u(t-\rho(t))) & -G\left(u_{\infty}\right)\left\|_{L^{2}}\right\| w(t) \|_{L^{2}} \\
& \leq 2 e^{\lambda t} L\left\|u(t-\rho(t))-u_{\infty}\right\|_{L^{2}}\|w(t)\|_{L^{2}} \\
& \leq e^{\lambda t}\left(L\left\|u(t-\rho(t))-u_{\infty}\right\|_{L^{2}}^{2}+L\|w(t)\|_{L^{2}}^{2}\right) \\
& \leq e^{\lambda t}\left(L\|w(t-\rho(t))\|_{L^{2}}^{2}+\frac{L}{\lambda_{1}}\|w(t)\|_{V}^{2}\right)
\end{aligned}
$$

e também,

$$
\begin{aligned}
2 e^{\lambda t} b(w(t & \left.-\tau(t)), w(t), u_{\infty}\right) \\
& \leq 2 e^{\lambda t}\|w(t-\tau(t))\|_{L^{4}}\|\nabla w(t)\|_{L^{2}}\left\|u_{\infty}\right\|_{L^{4}} \\
& \leq 2 e^{\lambda t} C_{1}\|w(t-\tau(t))\|_{V}\|w(t)\|_{V} C_{1}\left\|u_{\infty}\right\|_{V}
\end{aligned}
$$

onde $C_{1}$ é a constante de inclusão de $V$ em $\left(L^{4}(\Omega)\right)^{3}$. Chegamos à estimativa

$$
\begin{aligned}
\frac{d}{d t}\left(e^{\lambda t}\|w(t)\|_{L^{2}}^{2}\right) \leq & e^{\lambda t}\left(\frac{\lambda}{\lambda_{1}}-2 \nu+\frac{L}{\lambda_{1}}\right)\|w(t)\|_{V}^{2}+L e^{\lambda t}\|w(t-\rho(t))\|_{L^{2}}^{2} \\
& +2 e^{\lambda t} C_{1}\|w(t-\tau(t))\|_{V}\|w(t)\|_{V} C_{1}\left\|u_{\infty}\right\|_{V} .
\end{aligned}
$$

Por outro lado, aplicando a equação estacionária

$$
\nu A u_{\infty}+B\left(u_{\infty}, u_{\infty}\right)=f+G\left(u_{\infty}\right)
$$


em $u_{\infty}$, temos

$$
\begin{aligned}
\nu\left\|u_{\infty}\right\|_{V}^{2} & \leq\|f\|_{V^{\prime}}\left\|u_{\infty}\right\|_{V}+L\left\|u_{\infty}\right\|_{L^{2}}\left\|u_{\infty}\right\|_{L^{2}} \\
& \leq\|f\|_{V^{\prime}}\left\|u_{\infty}\right\|_{V}+\frac{L}{\lambda_{1}}\left\|u_{\infty}\right\|_{V}^{2}
\end{aligned}
$$

donde obtemos

$$
\left(\nu-\frac{L}{\lambda_{1}}\right)\left\|u_{\infty}\right\|_{V} \leq\|f\|_{V^{\prime}}
$$

ou ainda,

$$
\left\|u_{\infty}\right\|_{V} \leq \frac{\|f\|_{V^{\prime}}}{\left(\nu-\frac{L}{\lambda_{1}}\right)},
$$

e substituindo esta estimativa obtemos

$$
\begin{aligned}
\frac{d}{d t}\left(e^{\lambda t}\|w(t)\|_{L^{2}}^{2}\right) \leq & e^{\lambda t}\left(\frac{\lambda}{\lambda_{1}}-2 \nu+\frac{L}{\lambda_{1}}\right)\|w(t)\|_{V}^{2}+L e^{\lambda t}\|w(t-\rho(t))\|_{L^{2}}^{2} \\
& +2 e^{\lambda t} C_{1}\|w(t-\tau(t))\|_{V}\|w(t)\|_{V} \frac{C_{1}\|f\|_{V^{\prime}}}{\left(\nu-\frac{L}{\lambda_{1}}\right)} \\
\leq & e^{\lambda t}\left(\frac{\lambda}{\lambda_{1}}-2 \nu+\frac{L}{\lambda_{1}}\right)\|w(t)\|_{V}^{2}+L e^{\lambda t}\|w(t-\rho(t))\|_{L^{2}}^{2} \\
& +e^{\lambda t} C_{1}^{2}\|w(t-\tau(t))\|_{V}^{2}+e^{\lambda t}\left(\frac{C_{1}\|f\|_{V^{\prime}}}{\left(\nu-L \lambda_{1}^{-1}\right)}\right)^{2}\|w(t)\|_{V}^{2} \\
\leq & e^{\lambda t}\left(\frac{\lambda}{\lambda_{1}}-2 \nu+\frac{L}{\lambda_{1}}+\left(\frac{C_{1}\|f\|_{V^{\prime}}}{\left(\nu-L \lambda_{1}^{-1}\right)}\right)^{2}\right)\|w(t)\|_{V}^{2} \\
& +L e^{\lambda t}\|w(t-\rho(t))\|_{L^{2}}^{2}+e^{\lambda t} C_{1}^{2}\|w(t-\tau(t))\|_{V}^{2}
\end{aligned}
$$

Integrando em $t$, temos

$$
\begin{gathered}
e^{\lambda t}\|w(t)\|_{L^{2}}^{2} \\
\leq\|w(0)\|_{L^{2}}^{2}+\left(\frac{\lambda}{\lambda_{1}}-2 \nu+\frac{L}{\lambda_{1}}+\left(\frac{C_{1}\|f\|_{V^{\prime}}}{\left(\nu-L \lambda_{1}^{-1}\right)}\right)^{2}\right) \int_{0}^{t} e^{\lambda s}\|w(s)\|_{V}^{2} d s \\
+L \int_{0}^{t} e^{\lambda s}\|w(s-\rho(s))\|_{L^{2}}^{2} d s+C_{1}^{2} \int_{0}^{t} e^{\lambda s}\|w(s-\tau(s))\|_{V}^{2} d s \\
\leq\|w(0)\|_{L^{2}}^{2}+\left(\frac{\lambda}{\lambda_{1}}-2 \nu+\frac{L}{\lambda_{1}}+\left(\frac{C_{1}\|f\|_{V^{\prime}}}{\left(\nu-L \lambda_{1}^{-1}\right)}\right)^{2}\right) \int_{0}^{t} e^{\lambda s}\|w(s)\|_{V}^{2} d s \\
\quad+\frac{L e^{\lambda h}}{\left(1-\rho^{*}\right)} \int_{-h}^{t} e^{\lambda s}\|w(s)\|_{L^{2}}^{2} d s+\frac{e^{\lambda h} C_{1}^{2}}{\left(1-\tau^{*}\right)} \int_{-h}^{t} e^{\lambda s}\|w(s)\|_{V}^{2} d s \\
\leq\|w(0)\|_{L^{2}}^{2}+\left(\frac{L e^{\lambda h}}{\left(1-\rho^{*}\right) \lambda_{1}}+\frac{C_{1}^{2} e^{\lambda h}}{\left(1-\tau^{*}\right)}\right) \int_{-h}^{0} e^{\lambda s}\|w(s)\|_{V}^{2} d s \\
\quad+\left(\frac{\lambda}{\lambda_{1}}-2 \nu+\frac{L}{\lambda_{1}}+\left(\frac{C_{1}\|f\|_{V^{\prime}}}{\left(\nu-L \lambda_{1}^{-1}\right)}\right)^{2}+\frac{L e^{\lambda h}}{\left(1-\rho^{*}\right) \lambda_{1}}+\frac{C_{1}^{2} e^{\lambda h}}{\left(1-\tau^{*}\right)}\right) \int_{0}^{t} e^{\lambda s}\|w(s)\|_{V}^{2} d s .
\end{gathered}
$$


Em virtude da hipótese (3.14), podemos escolher $\lambda>0$ para que a constante da última integral seja igual a zero, e a desigualdade restante,

$$
\begin{aligned}
e^{\lambda t}\|w(t)\|_{L^{2}}^{2} & \leq\|w(0)\|_{L^{2}}^{2}+\left(\frac{L e^{\lambda h}}{\left(1-\rho^{*}\right) \lambda_{1}}+\frac{C_{1}^{2} e^{\lambda h}}{\left(1-\tau^{*}\right)}\right) \int_{-h}^{0} e^{\lambda s}\|w(s)\|_{V}^{2} d s \\
& \leq\|w(0)\|_{L^{2}}^{2}+\left(\frac{L e^{\lambda h}}{\left(1-\rho^{*}\right) \lambda_{1}}+\frac{C_{1}^{2} e^{\lambda h}}{\left(1-\tau^{*}\right)}\right)\|w(s)\|_{L^{2}(-h, 0 ; V)}^{2}
\end{aligned}
$$

nos leva a

$$
e^{\lambda t}\|w(t)\|_{L^{2}}^{2} \leq C\left(\|w(0)\|_{L^{2}}^{2}+\|w(s)\|_{L^{2}(-h, 0 ; V)}^{2}\right)
$$

e portanto

$$
\left\|u(t)-u_{\infty}\right\|_{L^{2}}^{2} \leq C e^{-\lambda t}\left(\left\|u_{0}-u_{\infty}\right\|_{L^{2}}^{2}+\left\|\phi-u_{\infty}\right\|_{L^{2}(-h, 0 ; V)}^{2}\right),
$$

e esta prova fica terminada.

\section{Apêndice}

Estamos agora interessados em garantir a existência de solução, em algum intervalo $(-h, T]$, para o problema aproximado (3.5). Para isto, estudaremos o problema

$$
\begin{aligned}
\frac{d}{d t} \Gamma_{k}(t) & =\Phi_{1}\left(\Gamma_{k}(t)\right)+\Phi_{2}\left(\Gamma_{k}(t-\tau(t)), \Gamma_{k}(t)\right) \\
+ & \Phi_{3}(f(t))+\Phi_{4}\left(g\left(t, \xi_{t}^{k}\right)\right), \\
\Gamma_{k}(0) & =u_{0}^{k}, \\
\Gamma_{k}(s) & =\phi_{k}(s) \quad \text { para } \quad s \in(-h, 0) .
\end{aligned}
$$

onde a função $\Gamma_{k}(t)=\left(\gamma_{1 k}(t), \gamma_{2 k}(t), \ldots, \gamma_{k k}(t)\right)$ é uma função vetorial em $\mathbb{R}^{k}, u_{0}^{k} \in \mathbb{R}^{k}$ e as funções $\Phi_{i}(i=1,2,3,4)$ e $\xi^{k}$ são também funções vetoriais em $\mathbb{R}^{k}$, que dependem de $\Gamma_{k} \mathrm{e}$ que satisfazem

$$
\Phi_{1}(0)=\Phi_{2}(0,0)=\Phi_{3}(0)=\Phi_{4}(0)=0, \quad \xi^{k}(0)=0,
$$

e as condições de Lipschitz,

$$
\begin{gathered}
\left\|\Phi_{1}\left(\Gamma_{k}(t)\right)-\Phi_{1}\left(\bar{\Gamma}_{k}(t)\right)\right\|_{k} \leq \mathcal{C}_{1}\left\|\Gamma_{k}(t)-\bar{\Gamma}_{k}(t)\right\|_{k}, \\
\left\|\Phi_{2}\left(\Gamma_{k}(t-\tau(t)), \Gamma_{k}(t)\right)-\Phi_{2}\left(\bar{\Gamma}_{k}(t-\tau(t)), \bar{\Gamma}_{k}(t)\right)\right\|_{k} \\
\leq \mathcal{C}_{2}\left\|\Gamma_{k}(t-\tau(t))-\bar{\Gamma}_{k}(t-\tau(t))\right\|_{k}\left\|\Gamma_{k}(t)\right\|_{k}+ \\
+\mathcal{C}_{2}\left\|\bar{\Gamma}_{k}(t-\tau(t))\right\|_{k}\left\|\Gamma_{k}(t)-\bar{\Gamma}_{k}(t)\right\|_{k}, \\
\left\|\Phi_{3}(f(t))\right\|_{k} \leq \mathcal{C}_{3}\|f(t)\|_{V^{\prime}}
\end{gathered}
$$

e

$$
\left\|\Phi_{4}\left(g\left(t, \xi_{t}^{k}\right)\right)-\Phi_{4}\left(g\left(t, \eta_{t}^{k}\right)\right)\right\|_{k} \leq \mathcal{C}_{4}\left\|g\left(t, \xi_{t}^{k}\right)-g\left(t, \eta_{t}^{k}\right)\right\|_{L^{2}}
$$


com

$$
\left\|\xi^{k}\left(\Gamma_{k}(s)\right)-\xi^{k}\left(\bar{\Gamma}_{k}(s)\right)\right\|_{V} \leq \mathcal{C}_{5}\left\|\Gamma_{k}(s)-\bar{\Gamma}_{k}(s)\right\|_{k}
$$

Além disso, a função $g$ satisfaz as hipóteses (H1)-(H5) e $f \in L^{2}\left([0, T], V^{\prime}\right)$.

Na busca por uma solução para a equação (3.20), procuramos uma solução para a equação integral,

$$
\begin{aligned}
\Gamma_{k}(t)=u_{0}^{k}+ & \int_{0}^{t} \Phi_{1}\left(\Gamma_{k}(s)\right) d s+\int_{0}^{t} \Phi_{2}\left(\Gamma_{k}(s-\tau(s)), \Gamma_{k}(s)\right) d s \\
& +\int_{0}^{t} \Phi_{3}(f(s)) d s+\int_{0}^{t} \Phi_{4}\left(g\left(s, \xi_{s}^{k}\right)\right) d s .
\end{aligned}
$$

Teorema 3.4.3. Sejam $u_{0} \in \mathbb{R}^{k}, \phi^{k} \in L^{2}\left(0, T ; \mathbb{R}^{k}\right), g:[0, T] \times C\left([0, T] ; \mathbb{R}^{k}\right) \rightarrow \mathbb{R}^{k}$ satisfazendo as hipóteses (H3) e (H4). Assumindo $\Phi_{1}, \Phi_{2}, \Phi_{3}, \Phi_{4}$ como acima, temos que

(i) existe $t_{*} \in[0, T]$, tal que o problema $(3.26)$,

$$
\begin{aligned}
\Gamma_{k}(t)=u_{0}^{k} & +\int_{0}^{t} \Phi_{1}\left(\Gamma_{k}(s)\right) d s+\int_{0}^{t} \Phi_{2}\left(\Gamma_{k}(s-\tau(s)), \Gamma_{k}(s)\right) d s \\
& \quad+\int_{0}^{t} \Phi_{3}(f(s)) d s+\int_{0}^{t} \Phi_{4}\left(g\left(s, \xi_{s}^{k}\right)\right) d s \quad t \in\left[0, t_{*}\right] \\
\Gamma_{k}(t)=\phi^{k}(t) \quad t \in(-h, 0) &
\end{aligned}
$$

possui uma única solução para todo $t \in\left[0, t_{*}\right]$.

(ii) Suponha que existe $C>0$ tal que, se $t_{*} \in(0, T]$ é tal que existe uma solução $\Gamma_{k}(t)$ para (3.26), então $\sup _{0 \leq t \leq t_{*}}\left\|\Gamma_{k}(t)\right\|_{k} \leq C$. Nestas condições existe uma solução para (3.26) com $t_{*}=T$.

Prova. Tomemos $C>0$ tal que $\left\|u_{0}^{k}\right\|_{k} \leq C$. Sejam

$$
M=C+2
$$

e $t_{*}>0$ satisfazendo

$$
\begin{gathered}
\left(\mathcal{C}_{1} M+\frac{\mathcal{C}_{2} M^{2}}{\left(1-\tau^{*}\right)^{\frac{1}{2}}}+\mathcal{C}_{4} C_{g}^{\frac{1}{2}} \mathcal{C}_{5}^{\frac{1}{2}} M\right) t_{*} \\
+\left(\frac{\mathcal{C}_{2} M}{\left(1-\tau^{*}\right)^{\frac{1}{2}}}\left\|\phi^{k}\right\|_{L^{2}\left(-h, 0 ; \mathbb{R}^{k}\right)}+\frac{\mathcal{C}_{2} M}{\left(1-\tau^{*}\right)^{\frac{1}{2}}} C h^{\frac{1}{2}}\right) t_{*}^{\frac{1}{2}} \\
+\left(\mathcal{C}_{3}\|f\|_{L^{2}\left(0, T ; V^{\prime}\right)}+\mathcal{C}_{4} C_{g}^{\frac{1}{2}} \mathcal{C}_{5}^{\frac{1}{2}}\left\|\phi^{k}\right\|_{L^{2}\left(-h, 0 ; \mathbb{R}^{k}\right)}+\mathcal{C}_{4} C_{g}^{\frac{1}{2}} \mathcal{C}_{5}^{\frac{1}{2}} C h^{\frac{1}{2}}\right) t_{*}^{\frac{1}{2}}<1,
\end{gathered}
$$

e sendo $\frac{T}{t_{*}}$ um inteiro. Definimos

$$
X=\left\{\Gamma \in L^{2}\left(-h, t_{*} ; \mathbb{R}^{k}\right) \cap C^{0}\left(\left[0, t_{*}\right] ; \mathbb{R}^{k}\right) ;\right.
$$




$$
\left.\Gamma=\phi^{k} \quad \text { em } \quad(-h, 0), \quad\|\Gamma(t)\|_{k} \leq M \quad \text { em } \quad\left[0, t_{*}\right]\right\}
$$

Dotemos $X$ da métrica

$$
d\left(\Gamma_{k}, \bar{\Gamma}_{k}\right)=\max _{t \in\left[0, t_{*}\right]}\left\|\Gamma_{k}(t)-\bar{\Gamma}_{k}(t)\right\|_{k}
$$

Claramente $(X, d)$ é um espaço métrico completo e $X \neq \emptyset$. Para cada $\Gamma_{k}$ em $X$, definimos

$$
\mathcal{T}\left(\Gamma_{k}\right)(t)=\left\{\begin{aligned}
\phi^{k}(t) \quad t \in(-h, 0) & \\
u_{0}^{k}+\int_{0}^{t} & \Phi_{1}\left(\Gamma_{k}(s)\right) d s+\int_{0}^{t} \Phi_{2}\left(\Gamma_{k}(s-\tau(s)), \Gamma_{k}(s)\right) d s \\
& \quad+\int_{0}^{t} \Phi_{3}(f(s)) d s+\int_{0}^{t} \Phi_{4}\left(g\left(s, \xi_{s}^{k}\right)\right) d s \quad t \in\left[0, t_{*}\right] .
\end{aligned}\right.
$$

Nestes termos $\mathcal{T}\left(\Gamma_{k}\right) \in L^{2}\left(-h, t_{*} ; \mathbb{R}^{k}\right) \cap C^{0}\left(\left[0, t_{*}\right] ; \mathbb{R}^{k}\right)$ e por definição, $\mathcal{T}\left(\Gamma_{k}\right)=\phi^{k}$ em $(-h, 0)$. Para cada $0 \leq t \leq t_{*}$ temos

$$
\begin{gathered}
\left\|\mathcal{T}\left(\Gamma_{k}\right)(t)\right\|_{k} \leq\left\|u_{0}^{k}\right\|_{k}+\int_{0}^{t}\left\|\Phi_{1}\left(\Gamma_{k}(s)\right)\right\|_{k} d s+\int_{0}^{t}\left\|\Phi_{2}\left(\Gamma_{k}(s-\tau(s)), \Gamma_{k}(s)\right)\right\|_{k} d s \\
\quad+\int_{0}^{t}\left\|\Phi_{3}(f(s))\right\|_{k} d s+\int_{0}^{t}\left\|\Phi_{4}\left(g\left(s, \xi_{s}^{k}\right)\right)\right\|_{k} d s \\
\leq C+\mathcal{C}_{1} \int_{0}^{t}\left\|\Gamma_{k}(s)\right\|_{k} d s+\mathcal{C}_{2} \int_{0}^{t}\left\|\Gamma_{k}(s-\tau(s))\right\|_{k}\left\|\Gamma_{k}(s)\right\|_{k} d s \\
+\mathcal{C}_{3} \int_{0}^{t}\|f(s)\|_{V^{\prime}} d s+\mathcal{C}_{4} \int_{0}^{t}\left\|g\left(s, \xi_{s}^{k}\right)\right\|_{L^{2}} d s \\
\leq C+\mathcal{C}_{1} M t_{*}+\mathcal{C}_{2}\left(\int_{0}^{t}\left\|\Gamma_{k}(s)\right\|_{k}^{2} d s\right)^{\frac{1}{2}}\left(\int_{0}^{t}\left\|\Gamma_{k}(s-\tau(s))\right\|_{k}^{2} d s\right)^{\frac{1}{2}} \\
+\mathcal{C}_{3}\left(\int_{0}^{t} 1 d s\right)^{\frac{1}{2}}\left(\int_{0}^{t}\|f(s)\|_{V^{\prime}}^{2} d s\right)^{\frac{1}{2}}+\mathcal{C}_{4} \int_{0}^{t}\left\|g\left(s, \xi_{s}^{k}\right)\right\|_{L^{2}} d s \\
\leq C+\mathcal{C}_{1} M t_{*}+\mathcal{C}_{2} t_{*}^{\frac{1}{2}} M\left(\int_{0}^{t}\left\|\Gamma_{k}(s-\tau(s))\right\|_{k}^{2} d s\right)^{\frac{1}{2}} \\
+\mathcal{C}_{3} t_{*}^{\frac{1}{2}}\|f\|_{L^{2}\left(0, T ; V^{\prime}\right)}+\mathcal{C}_{4} \int_{0}^{t}\left\|g\left(s, \xi_{s}^{k}\right)\right\|_{L^{2}} d s \\
\leq C+\mathcal{C}_{1} M t_{*}+\frac{\mathcal{C}_{2} t_{*}^{\frac{1}{2}} M}{\left(1-\tau^{*}\right)^{\frac{1}{2}}}\left(\int_{-h}^{t}\left\|\Gamma_{k}(s)\right\|_{k}^{2} d s\right)^{\frac{1}{2}} \\
+\mathcal{C}_{3} t_{*}^{\frac{1}{2}}\|f\|_{L^{2}\left(0, T ; V^{\prime}\right)}+\mathcal{C}_{4} \int_{0}^{t}\left\|g\left(s, \xi_{s}^{k}\right)\right\|_{L^{2}} d s .
\end{gathered}
$$

Agora, como

$$
\begin{aligned}
\left(\int_{-h}^{t}\left\|\Gamma_{k}(s)\right\|_{k}^{2} d s\right)^{\frac{1}{2}} & =\left(\int_{-h}^{0}\left\|\Gamma_{k}(s)\right\|_{k}^{2} d s+\int_{0}^{t}\left\|\Gamma_{k}(s)\right\|_{k}^{2} d s\right)^{\frac{1}{2}} \\
& \leq\left(\int_{-h}^{0}\left\|\phi^{k}(s)\right\|_{k}^{2} d s+M^{2} t_{*}\right)^{\frac{1}{2}} \\
& =\left(\left\|\phi^{k}\right\|_{L^{2}\left(-h, 0 ; \mathbb{R}^{k}\right)}^{2}+M^{2} t_{*}\right)^{\frac{1}{2}}
\end{aligned}
$$




$$
\begin{aligned}
& \leq\left\|\phi^{k}\right\|_{L^{2}\left(-h, 0 ; \mathbb{R}^{k}\right)}+M t_{*}^{\frac{1}{2}} \\
& \leq\left\|\phi^{k}\right\|_{L^{2}\left(-h, 0 ; \mathbb{R}^{k}\right)}+C h^{\frac{1}{2}}+M t_{*}^{\frac{1}{2}}
\end{aligned}
$$

temos

$$
\begin{aligned}
\left\|\mathcal{T}\left(\Gamma_{k}\right)(t)\right\|_{k} \leq C & +\mathcal{C}_{1} M t_{*}+\frac{\mathcal{C}_{2} M t_{*}^{\frac{1}{2}}}{\left(1-\tau^{*}\right)^{\frac{1}{2}}}\left\|\phi^{k}\right\|_{L^{2}\left(-h, 0 ; \mathbb{R}^{k}\right)}+\frac{\mathcal{C}_{2} M C h^{\frac{1}{2}} t_{*}^{\frac{1}{2}}}{\left(1-\tau^{*}\right)^{\frac{1}{2}}} \\
& +\frac{\mathcal{C}_{2} t_{*} M^{2}}{\left(1-\tau^{*}\right)^{\frac{1}{2}}}+\mathcal{C}_{3} t_{*}^{\frac{1}{2}}\|f\|_{L^{2}\left(0, T ; V^{\prime}\right)}+\mathcal{C}_{4} \int_{0}^{t}\left\|g\left(s, \xi_{s}^{k}\right)\right\|_{L^{2}} d s .
\end{aligned}
$$

Vamos agora estimar a integral restante do segundo membro. Usando a desigualdade (H4) temos

$$
\begin{aligned}
\mathcal{C}_{4} \int_{0}^{t}\left\|g\left(s, \xi_{s}^{k}\right)\right\|_{L^{2}} d s & \leq \mathcal{C}_{4}\left(\int_{0}^{t} 1 d s\right)^{\frac{1}{2}}\left(\int_{0}^{t}\left\|g\left(s, \xi_{s}^{k}\right)\right\|_{L^{2}}^{2} d s\right)^{\frac{1}{2}} \\
& \leq \mathcal{C}_{4} t_{*}^{\frac{1}{2}}\left(\int_{0}^{t}\left\|g\left(s, \xi_{s}^{k}\right)\right\|_{L^{2}}^{2} d s\right)^{\frac{1}{2}} \\
& \leq \mathcal{C}_{4} t_{*}^{\frac{1}{2}} C_{g}^{\frac{1}{2}}\left(\int_{-h}^{t}\left\|\xi^{k}(s)\right\|_{V}^{2} d s\right)^{\frac{1}{2}} \\
& \leq \mathcal{C}_{4} t_{*}^{\frac{1}{2}} C_{g}^{\frac{1}{2}}\left(\int_{-h}^{0}\left\|\xi^{k}(s)\right\|_{V}^{2} d s+\int_{0}^{t}\left\|\xi^{k}(s)\right\|_{V}^{2} d s\right)^{\frac{1}{2}} \\
& \leq \mathcal{C}_{4} t_{*}^{\frac{1}{2}} C_{g}^{\frac{1}{2}}\left(\mathcal{C}_{5} \int_{-h}^{0}\left\|\Gamma_{k}(s)\right\|_{k}^{2} d s+\mathcal{C}_{5} \int_{0}^{t}\left\|\Gamma_{k}(s)\right\|_{k}^{2} d s\right)^{\frac{1}{2}} \\
& \leq \mathcal{C}_{4} t_{*}^{\frac{1}{2}} C_{g}^{\frac{1}{2}}\left(\mathcal{C}_{5}\left\|\phi^{k}\right\|_{L^{2}\left(-h, 0 ; \mathbb{R}^{k}\right)}^{2}+\mathcal{C}_{5} M^{2} t_{*}\right)^{\frac{1}{2}} \\
& \leq \mathcal{C}_{4} t_{*}^{\frac{1}{2}} C_{g}^{\frac{1}{2}} \mathcal{C}_{5}^{\frac{1}{2}}\left(\left\|\phi^{k}\right\|_{L^{2}\left(-h, 0 ; \mathbb{R}^{k}\right)}+M t_{*}^{\frac{1}{2}}\right) \\
& \leq \mathcal{C}_{4} t_{*}^{\frac{1}{2}} C_{g}^{\frac{1}{2}} \mathcal{C}_{5}^{\frac{1}{2}}\left(\left\|\phi^{k}\right\|_{L^{2}\left(-h, 0 ; \mathbb{R}^{k}\right)}+C h^{\frac{1}{2}}+M t_{*}^{\frac{1}{2}}\right) \\
& =\mathcal{C}_{4} t_{*}^{\frac{1}{2}} C_{g}^{\frac{1}{2}} \mathcal{C}_{5}^{\frac{1}{2}}\left\|\phi^{k}\right\|_{L^{2}\left(-h, 0 ; \mathbb{R}^{k}\right)}+\mathcal{C}_{4} C h^{\frac{1}{2}} t_{*}^{\frac{1}{2}} C_{g}^{\frac{1}{2}} \mathcal{C}_{5}^{\frac{1}{2}}+\mathcal{C}_{4} C_{g}^{\frac{1}{2}} \mathcal{C}_{5}^{\frac{1}{2}} M t_{*} .
\end{aligned}
$$

Voltando então para a limitação de $\mathcal{T}\left(\Gamma_{k}\right)$, temos

$$
\begin{aligned}
\left\|\mathcal{T}\left(\Gamma_{k}\right)(t)\right\|_{k} \leq C+\mathcal{C}_{1} M t_{*}+\frac{\mathcal{C}_{2} M t_{*}^{\frac{1}{2}}}{\left(1-\tau^{*}\right)^{\frac{1}{2}}}\left\|\phi^{k}\right\|_{L^{2}\left(-h, 0 ; \mathbb{R}^{k}\right)}+\frac{\mathcal{C}_{2} M C h^{\frac{1}{2}} t_{*}^{\frac{1}{2}}}{\left(1-\tau^{*}\right)^{\frac{1}{2}}} \\
+\frac{\mathcal{C}_{2} t_{*} M^{2}}{\left(1-\tau^{*}\right)^{\frac{1}{2}}}+\mathcal{C}_{3} t_{*}^{\frac{1}{2}}\|f\|_{L^{2}\left(0, T ; V^{\prime}\right)} \\
+\mathcal{C}_{4} t_{*}^{\frac{1}{2}} C_{g}^{\frac{1}{2}} \mathcal{C}_{5}^{\frac{1}{2}}\left\|\phi^{k}\right\|_{L^{2}\left(-h, 0 ; \mathbb{R}^{k}\right)}+\mathcal{C}_{4} C h^{\frac{1}{2}} t_{*}^{\frac{1}{2}} C_{g}^{\frac{1}{2}} \mathcal{C}_{5}^{\frac{1}{2}}+\mathcal{C}_{4} C_{g}^{\frac{1}{2}} \mathcal{C}_{5}^{\frac{1}{2}} M t_{*}
\end{aligned}
$$

e pela escolha de $t_{*}$ e de $M$, temos

$$
\left\|\mathcal{T}\left(\Gamma_{k}\right)(t)\right\|_{k} \leq C+1<C+2=M
$$


o que garante que $\mathcal{T}$ leva $X$ em $X$. Mostraremos agora que $\mathcal{T}$ é contração. De fato, se $\Gamma_{k} \mathrm{e}$ $\bar{\Gamma}_{k}$ pertencem a $X$, então

$$
\begin{aligned}
& d\left(\mathcal{T}\left(\Gamma_{k}\right), \mathcal{T}\left(\bar{\Gamma}_{k}\right)\right)=\max _{0 \leq t \leq t_{*}}\left\|\mathcal{T}\left(\Gamma_{k}\right)(t)-\mathcal{T}\left(\bar{\Gamma}_{k}\right)(t)\right\|_{k} \\
& \leq \int_{0}^{t_{*}}\left\|\Phi_{1}\left(\Gamma_{k}(s)\right)-\Phi_{1}\left(\bar{\Gamma}_{k}(s)\right)\right\|_{k} d s \\
& +\int_{0}^{t_{*}}\left\|\Phi_{2}\left(\Gamma_{k}(s-\tau(s)), \Gamma_{k}(s)\right)-\Phi_{2}\left(\bar{\Gamma}_{k}(s-\tau(s)), \bar{\Gamma}_{k}(s)\right)\right\|_{k} d s \\
& +\int_{0}^{t_{*}}\left\|\Phi_{4}\left(g\left(s, \xi_{s}^{k}\right)\right)-\Phi_{4}\left(g\left(s, \eta_{s}^{k}\right)\right)\right\|_{k} d s \\
& \leq \mathcal{C}_{1} \int_{0}^{t_{*}}\left\|\Gamma_{k}(s)-\bar{\Gamma}_{k}(s)\right\|_{k} d s \\
& +\mathcal{C}_{2} \int_{0}^{t_{*}}\left\|\Gamma_{k}(s-\tau(s))-\bar{\Gamma}_{k}(s-\tau(s))\right\|\left\|_{k}\right\| \Gamma_{k}(s) \|_{k} d s \\
& +\mathcal{C}_{2} \int_{0}^{t_{*}}\left\|\Gamma_{k}(s)-\bar{\Gamma}_{k}(s)\right\|_{k}\left\|\bar{\Gamma}_{k}(s-\tau(s))\right\|_{k} d s \\
& +\mathcal{C}_{4} \int_{0}^{t_{*}}\left\|g\left(s, \xi_{s}^{k}\right)-g\left(s, \eta_{s}^{k}\right)\right\|_{L^{2}} d s \\
& \leq \mathcal{C}_{1} \int_{0}^{t_{*}}\left\|\Gamma_{k}(s)-\bar{\Gamma}_{k}(s)\right\|_{k} d s \\
& +\mathcal{C}_{2}\left(\int_{0}^{t}\left\|\Gamma_{k}(s)\right\|_{k}^{2} d s\right)^{\frac{1}{2}}\left(\int_{0}^{t_{*}}\left\|\Gamma_{k}(s-\tau(s))-\bar{\Gamma}_{k}(s-\tau(s))\right\|_{k}^{2} d s\right)^{\frac{1}{2}} \\
& +\mathcal{C}_{2}\left(\int_{0}^{t}\left\|\Gamma_{k}(s)-\bar{\Gamma}_{k}(s)\right\|_{k}^{2} d s\right)^{\frac{1}{2}}\left(\int_{0}^{t_{*}}\left\|\bar{\Gamma}_{k}(s-\tau(s))\right\|_{k}^{2} d s\right)^{\frac{1}{2}} \\
& +\mathcal{C}_{4}\left(\int_{0}^{t} 1 d s\right)^{\frac{1}{2}}\left(\int_{0}^{t_{*}}\left\|g\left(s, \xi_{s}^{k}\right)-g\left(s, \eta_{s}^{k}\right)\right\|_{L^{2}}^{2} d s\right)^{\frac{1}{2}} \\
& \leq \mathcal{C}_{1} t_{*} d\left(\Gamma_{k}, \bar{\Gamma}_{k}\right)+\frac{\mathcal{C}_{2} M t_{*}^{\frac{1}{2}}}{\left(1-\tau^{*}\right)^{\frac{1}{2}}}\left(\int_{0}^{t_{*}}\left\|\Gamma_{k}(s)-\bar{\Gamma}_{k}(s)\right\|_{k}^{2} d s\right)^{\frac{1}{2}} \\
& +\mathcal{C}_{2} t_{*}^{\frac{1}{2}} d\left(\Gamma_{k}, \bar{\Gamma}_{k}\right)\left(\int_{0}^{t_{*}}\left\|\bar{\Gamma}_{k}(s-\tau(s))\right\|_{k}^{2} d s\right)^{\frac{1}{2}} \\
& +\mathcal{C}_{4} C_{g}^{\frac{1}{2}} t_{*}^{\frac{1}{2}}\left(\int_{-h}^{t_{*}}\left\|\xi^{k}(s)-\eta^{k}(s)\right\|_{V}^{2} d s\right)^{\frac{1}{2}} \\
& \leq \mathcal{C}_{1} t_{*} d\left(\Gamma_{k}, \bar{\Gamma}_{k}\right)+\frac{\mathcal{C}_{2} M t_{*}}{\left(1-\tau^{*}\right)^{\frac{1}{2}}} d\left(\Gamma_{k}, \bar{\Gamma}_{k}\right) \\
& +\frac{\mathcal{C}_{2} t_{*}^{\frac{1}{2}}}{\left(1-\tau^{*}\right)^{\frac{1}{2}}} d\left(\Gamma_{k}, \bar{\Gamma}_{k}\right)\left(\int_{-h}^{t_{*}}\left\|\bar{\Gamma}_{k}(s)\right\|_{k}^{2} d s\right)^{\frac{1}{2}} \\
& +\mathcal{C}_{4} C_{g}^{\frac{1}{2}} t_{*}^{\frac{1}{2}} \mathcal{C}_{5}^{\frac{1}{2}}\left(\int_{0}^{t_{*}}\left\|\Gamma_{k}(s)-\bar{\Gamma}_{k}(s)\right\|_{k}^{2} d s\right)^{\frac{1}{2}} \\
& \leq\left(\mathcal{C}_{1} t_{*}+\frac{\mathcal{C}_{2} M t_{*}}{\left(1-\tau^{*}\right)^{\frac{1}{2}}}+\mathcal{C}_{4} C_{g}^{\frac{1}{2}} t_{*} \mathcal{C}_{5}^{\frac{1}{2}}\right) d\left(\Gamma_{k}, \bar{\Gamma}_{k}\right)
\end{aligned}
$$




$$
\begin{gathered}
\quad+\frac{\mathcal{C}_{2} t_{*}^{\frac{1}{2}}}{\left(1-\tau^{*}\right)^{\frac{1}{2}}} d\left(\Gamma_{k}, \bar{\Gamma}_{k}\right)\left(\int_{-h}^{0}\left\|\bar{\Gamma}_{k}(s)\right\|_{k}^{2} d s+\int_{0}^{t_{*}}\left\|\bar{\Gamma}_{k}(s)\right\|_{k}^{2} d s\right)^{\frac{1}{2}} \\
\leq\left(\mathcal{C}_{1} t_{*}+\frac{\mathcal{C}_{2} M t_{*}}{\left(1-\tau^{*}\right)^{\frac{1}{2}}}+\mathcal{C}_{4} C_{g}^{\frac{1}{2}} t_{*} \mathcal{C}_{5}^{\frac{1}{2}}\right) d\left(\Gamma_{k}, \bar{\Gamma}_{k}\right) \\
\quad+\frac{\mathcal{C}_{2} t_{*}^{\frac{1}{2}}}{\left(1-\tau^{*}\right)^{\frac{1}{2}}} d\left(\Gamma_{k}, \bar{\Gamma}_{k}\right)\left(\left\|\phi^{k}\right\|_{L^{2}\left(-h, 0 ; \mathbb{R}^{k}\right)}+M t_{*}^{\frac{1}{2}}\right) \\
=\left(\mathcal{C}_{1} t_{*}+\frac{2 \mathcal{C}_{2} M t_{*}}{\left(1-\tau^{*}\right)^{\frac{1}{2}}}+\mathcal{C}_{4} C_{g}^{\frac{1}{2}} t_{*} \mathcal{C}_{5}^{\frac{1}{2}}+\frac{\mathcal{C}_{2} t_{*}^{\frac{1}{2}}\left\|\phi^{k}\right\|_{L^{2}\left(-h, 0 ; \mathbb{R}^{k}\right)}}{\left(1-\tau^{*}\right)^{\frac{1}{2}}}\right) d\left(\Gamma_{k}, \bar{\Gamma}_{k}\right) .
\end{gathered}
$$

Agora, como $M>2>1$ então devido a escolha de $t_{*}$ temos

$$
\begin{aligned}
K & =\mathcal{C}_{1} t_{*}+\frac{2 \mathcal{C}_{2} M t_{*}}{\left(1-\tau^{*}\right)^{\frac{1}{2}}}+\mathcal{C}_{4} C_{g}^{\frac{1}{2}} t_{*} \mathcal{C}_{5}^{\frac{1}{2}}+\frac{\mathcal{C}_{2} t_{*}^{\frac{1}{2}}\left\|\phi^{k}\right\|_{L^{2}\left(-h, 0 ; \mathbb{R}^{k}\right)}}{\left(1-\tau^{*}\right)^{\frac{1}{2}}} \\
& \leq \mathcal{C}_{1} M t_{*}+\frac{\mathcal{C}_{2} M^{2} t_{*}}{\left(1-\tau^{*}\right)^{\frac{1}{2}}}+\mathcal{C}_{4} C_{g}^{\frac{1}{2}} \mathcal{C}_{5}^{\frac{1}{2}} M t_{*}+\frac{\mathcal{C}_{2} M\left\|\phi^{k}\right\|_{L^{2}\left(-h, 0 ; \mathbb{R}^{k}\right)}}{\left(1-\tau^{*}\right)^{\frac{1}{2}}} t_{*}^{\frac{1}{2}}<1
\end{aligned}
$$

o que garante que $\mathcal{T}$ é uma contração de $X$ em $X$. Segue agora do teorema de ponto fixo para contrações que $\mathcal{T}$ tem um único ponto fixo. Tal ponto fixo é a solução de (3.26), e portanto da equação (3.20) no intervalo desejado. Isto termina a prova de $(i)$.

Para provarmos $(i i)$, seja $C>0$ como na hipótese, isto é, se $t_{*}$ é tal que existe uma solução $\Gamma_{k}(t)$ de $(3.20)$ então

$$
\max _{0 \leq t \leq t_{*}}\left\|\Gamma_{k}(t)\right\|_{k} \leq C
$$

Tomemos $M$ e $t_{*}$ como no item $(i)$, e $\frac{T}{t_{*}}$ sendo um inteiro. Nestes termos, existe uma única $\Gamma_{k} \in L^{2}\left(-h, t_{*} ; \mathbb{R}^{k}\right) \cap C^{0}\left(\left[0, t_{*}\right] ; \mathbb{R}^{k}\right)$ tal que

$$
\begin{aligned}
\Gamma_{k}(t) & =\phi^{k}(t) \quad t \in(-h, 0) \\
\Gamma_{k}(t) & =u_{0}^{k}+\int_{0}^{t} \Phi_{1}\left(\Gamma_{k}(s)\right) d s+\int_{0}^{t} \Phi_{2}\left(\Gamma_{k}(s-\tau(s)), \Gamma_{k}(s)\right) d s \\
& +\int_{0}^{t} \Phi_{3}(f(s)) d s+\int_{0}^{t} \Phi_{4}\left(g\left(s, \xi_{s}^{k}\right)\right) d s .
\end{aligned}
$$

Se $t_{*}<T$, definimos,

$$
\widetilde{\phi}^{k}(s)=\Gamma_{k}\left(s+t_{*}\right) \quad \text { para } \quad s \in(-h, 0)
$$

e consideremos o problema de encontrar $\widetilde{\Gamma}_{k} \in X$ tal que

$$
\begin{aligned}
\widetilde{\Gamma}_{k}(t)= & \widetilde{\phi}^{k}(t) \quad t \in(-h, 0) \\
\widetilde{\Gamma}_{k}(t)= & \Gamma_{k}\left(t_{*}\right)+\int_{0}^{t} \Phi_{1}\left(\widetilde{\Gamma}_{k}(s)\right) d s+\int_{0}^{t} \Phi_{2}\left(\widetilde{\Gamma}_{k}(s-\tau(s)), \widetilde{\Gamma}_{k}(s)\right) d s \\
& \quad+\int_{0}^{t} \Phi_{3}(f(s)) d s+\int_{0}^{t} \Phi_{4}\left(g\left(s, \widetilde{\xi}_{s}^{k}\right)\right) d s \quad t \in\left[0, t_{*}\right] .
\end{aligned}
$$


Notemos que se $\left(t_{*}-h\right) \geq 0$, então

$$
\begin{aligned}
\int_{-h}^{0}\left\|\widetilde{\phi}^{k}(s)\right\|_{k}^{2} d s & =\int_{-h}^{0}\left\|\Gamma_{k}\left(s+t_{*}\right)\right\|_{k}^{2} d s \\
& =\int_{-h+t_{*}}^{t_{*}}\left\|\Gamma_{k}(s)\right\|_{k}^{2} d s \\
& \leq C^{2} \int_{-h+t_{*}}^{t_{*}} d s=C^{2} h \leq\left\|\phi^{k}\right\|_{L^{2}\left(-h, 0 ; \mathbb{R}^{k}\right)}^{2}+h C^{2},
\end{aligned}
$$

e por outro lado, se $\left(t_{*}-h\right)<0$, então

$$
\begin{aligned}
\int_{-h}^{0}\left\|\widetilde{\phi}^{k}(s)\right\|_{k}^{2} d s & =\int_{-h+t_{*}}^{t_{*}}\left\|\Gamma_{k}(s)\right\|_{k}^{2} d s \\
& \leq \int_{-h}^{0}\left\|\phi^{k}\right\|_{k}^{2} d s+\int_{0}^{t_{*}}\left\|\Gamma_{k}(s)\right\|_{k}^{2} d s \\
& \leq\left\|\phi^{k}\right\|_{L^{2}\left(-h, 0 ; \mathbb{R}^{k}\right)}^{2}+t_{*} C^{2} \leq\left\|\phi^{k}\right\|_{L^{2}\left(-h, 0 ; \mathbb{R}^{k}\right)}^{2}+h C^{2},
\end{aligned}
$$

donde $\left\|\widetilde{\phi}^{k}\right\|_{L^{2}\left(-h, 0 ; \mathbb{R}^{k}\right)}^{2} \leq\left\|\phi^{k}\right\|_{L^{2}\left(-h, 0 ; \mathbb{R}^{k}\right)}^{2}+h C^{2}$, e portanto

$$
\left\|\widetilde{\phi}^{k}\right\|_{L^{2}\left(-h, 0 ; \mathbb{R}^{k}\right)} \leq\left\|\phi^{k}\right\|_{L^{2}\left(-h, 0 ; \mathbb{R}^{k}\right)}+h^{\frac{1}{2}} C .
$$

O problema (3.27) tem a mesma estrutura do problema (3.26). As funções $\Phi_{1}, \Phi_{2}, \Phi_{3}$ e $\Phi_{4}$ possuem em (3.27) as mesmas estimativas que em (3.26). Vamos verificar isto. Para $\Phi_{1}$, temos como anteriormente,

$$
\left\|\Phi_{1}\left(\widetilde{\Gamma}_{k}(s)\right)-\Phi_{1}\left(\overline{\widetilde{\Gamma}}_{k}(s)\right)\right\|_{k} \leq \mathcal{C}_{1}\left\|\widetilde{\Gamma}_{k}(s)-\overline{\widetilde{\Gamma}}_{k}(s)\right\|_{k}
$$

donde

$$
\int_{0}^{t}\left\|\Phi_{1}(\widetilde{\Gamma}(s))\right\|_{k} d s \leq \mathcal{C}_{1} \int_{0}^{t}\|\widetilde{\Gamma}(s)\|_{k} d s \leq \mathcal{C}_{1} M t_{*}
$$

Para $\Phi_{2}$ temos

$$
\begin{aligned}
\| \Phi_{2}\left(\widetilde{\Gamma}_{k}(s-\tau(s)), \widetilde{\Gamma}_{k}(s)\right)- & \Phi_{2}\left(\widetilde{\widetilde{\Gamma}}_{k}(s-\tau(s)), \widetilde{\widetilde{\Gamma}}_{k}(s)\right) \|_{k} \\
\leq & \mathcal{C}_{2}\left\|\widetilde{\Gamma}_{k}(s-\tau(s))-\overline{\widetilde{\Gamma}}_{k}(s-\tau(s))\right\|_{k}\left\|\widetilde{\Gamma}_{k}(s)\right\|_{k} \\
& \quad+\mathcal{C}_{2}\left\|\widetilde{\Gamma}_{k}(s)-\overline{\widetilde{\Gamma}}_{k}(s)\right\|_{k}\left\|\overline{\widetilde{\Gamma}}_{k}(s-\tau(s))\right\|_{k}
\end{aligned}
$$

e da mesma forma que antes

$$
\begin{aligned}
\int_{0}^{t} \| \Phi_{2}\left(\widetilde{\Gamma}_{k}(s\right. & \left.-\tau(s)), \widetilde{\Gamma}_{k}(s)\right) \|_{k} d s \\
& \leq \frac{\mathcal{C}_{2} M t_{*}^{\frac{1}{2}}}{\left(1-\tau^{*}\right)^{\frac{1}{2}}}\left\|\widetilde{\phi}^{k}\right\|_{L^{2}\left(-h, 0 ; \mathbb{R}^{k}\right)}+\frac{\mathcal{C}_{2} M^{2} t_{*}}{\left(1-\tau^{*}\right)^{\frac{1}{2}}} \\
& \leq \frac{\mathcal{C}_{2} M t_{*}^{\frac{1}{2}}}{\left(1-\tau^{*}\right)^{\frac{1}{2}}}\left\|\phi^{k}\right\|_{L^{2}\left(-h, 0 ; \mathbb{R}^{k}\right)}+\frac{\mathcal{C}_{2} M t_{*}^{\frac{1}{2}} C h^{\frac{1}{2}}}{\left(1-\tau^{*}\right)^{\frac{1}{2}}}+\frac{\mathcal{C}_{2} M^{2} t_{*}}{\left(1-\tau^{*}\right)^{\frac{1}{2}}}
\end{aligned}
$$


Para $\Phi_{3}$, claramente segue a mesma limitação já que $\Phi_{3}$ não depende de $\widetilde{\Gamma}$, mas sim de $f \in L^{2}\left(0, T ; V^{\prime}\right)$. Finalmente, temos

$$
\left\|\Phi_{4}\left(g\left(s, \widetilde{\xi}_{s}^{k}\right)\right)-\Phi_{4}\left(g\left(s, \widetilde{\eta}_{s}^{k}\right)\right)\right\|_{k} \leq \mathcal{C}_{4}\left\|g\left(s, \widetilde{\xi}_{s}^{k}\right)-g\left(s, \widetilde{\eta}_{s}^{k}\right)\right\|_{L^{2}},
$$

e similarmente,

$$
\begin{aligned}
\int_{0}^{t}\left\|\Phi_{4}\left(g\left(s, \widetilde{\xi}_{s}^{k}\right)\right)\right\|_{k} d s & \leq \mathcal{C}_{4} C_{g}^{\frac{1}{2}} t_{*}^{\frac{1}{2}} \mathcal{C}_{5}^{\frac{1}{2}}\left\|\widetilde{\phi}^{k}\right\|_{L^{2}\left(-h, 0 ; \mathbb{R}^{k}\right)}+\mathcal{C}_{4} C_{g}^{\frac{1}{2}} M t_{*} \mathcal{C}_{5}^{\frac{1}{2}} \\
& \leq \mathcal{C}_{4} C_{g}^{\frac{1}{2}} t_{*}^{\frac{1}{2}} \mathcal{C}_{5}^{\frac{1}{2}}\left\|\phi^{k}\right\|_{L^{2}\left(-h, 0 ; \mathbb{R}^{k}\right)}+\mathcal{C}_{4} C_{g}^{\frac{1}{2}} t_{*}^{\frac{1}{2}} \mathcal{C}_{5}^{\frac{1}{2}} C h^{\frac{1}{2}}+\mathcal{C}_{4} C_{g}^{\frac{1}{2}} M t_{*} \mathcal{C}_{5}^{\frac{1}{2}}
\end{aligned}
$$

Procedendo como na parte $(i)$, podemos garantir a existência de uma única solução $\widetilde{\Gamma}_{k}(t)$, para (3.27), no intervalo $\left[0, t_{*}\right]$. Colocando então

$$
\boldsymbol{\Gamma}_{k}(t)=\left\{\begin{array}{l}
\Gamma_{k}(t) \quad t \in\left(-h, t_{*}\right] \\
\widetilde{\Gamma}_{k}\left(t-t_{*}\right) \quad t \in\left[t_{*}, 2 t_{*}\right]
\end{array}\right.
$$

e temos uma única solução para o problema (3.26) no intervalo $\left(-h, 2 t_{*}\right]$. Repetindo este processo, após um número finito de passos, obteremos uma única solução para (3.26) no intervalo $(-h, T]$. 


\section{Referências}

[1] Bridges, Thomas J. The Hopf bifurcation with symmetry for the Navier-Stokes Equations in $\left(L_{p}(\Omega)\right)^{n}$, with application to Plane Poiseuille Flow. Arch. Rational Mech. Anal. 106 (1989), pp. 335-376.

[2] Caraballo, Tomás \& Real, José. Navier-Stokes equations with delays. Proc. R. Soc. Lond. A 457 (2001), pp. 2441-2453.

[3] Caraballo, Tomás \& Real, José. Asymptotic behavior of two-dimensional NavierStokes equations with delays. Proc. R. Soc. Lond. A 459 (2003), pp. 3181-3194.

[4] Caraballo, Tomás \& Real, José. Attractors for $2 D$ Navier-Stokes models with delay. Journal of differential equations 205 (2004), no. 2, pp. 271-297.

[5] Cattabriga, Lamberto. Su un problema al contorno relativo al sistema di equazioni di Stokes. (Italian) Rend. Sem. Mat. Univ. Padova 311961 308-340.

[6] Constantin, Peter \& Foias, Ciprian. Navier-Stokes equations. University of Chicago Press, Chicago, 1988.

[7] Cheban, David \& Duan, Jinqiao. Almost periodic solutions and global attractors of non-autonomous Navier-Stokes equations. Journal of dynamics and differential equations. Vol. 16, No. 1, January 2004.

[8] Fujita, Hiroshi \& Kato, Tosio. On the Navier-Stokes initial value problem I. Arch. Rational Mech. Anal. 16 (1964) pp. 269-315.

[9] Fujiwara, D. \& Morimoto, H. An $L_{p}$ theorem of the Helmholtz decomposition of vector fields. J. Fa. sci. Univ. Tokyo, Sect. Math. IA 24 (1977), pp. 685-700.

[10] Garrido-Atienza, Maria José \& Marín-Rubio, Pedro. Navier-Stokes equations with delays on unbounded domains. Nonlinear analisys 64 (2006), no. 5, pp. 1100-1118.

[11] Giga, Yoshikazu. Analyticity of the semi-group generated by the Stokes operator in $L_{p}$ spaces. Math. Z., 178 (1981), pp. 297-329. 
[12] Giga, Yoshikazu. Domains of fractional powers of the Stokes operator in $L_{p}$ spaces. Arch. Rational Mech. Anal. 89 (1985), pp. 251-265.

[13] Giga, Yoshikazu \& Miyakawa, Tetsuro. Solutions in $L_{p}$ of the Navier-Stokes initial value problem. Arch. Rational Mech. Anal. 89 (1985), pp. 267-281.

[14] Hino, Y.; Murakami, S.; Naito, T. Functional-differential equations with infinite delay. Lecture Notes in Mathematics 1473, Springer-Verlag, Berlin, 1991.

[15] Hopf, E. Über die Anfangswertaufgabe für die hydrodynamischen Grundgleichungen. Math. Nachr. 4 (1951), pp. 213-231.

[16] Kato, Hisako. Existence of periodic solutions of the Navier-Stokes equations. Journal of mathematical analysis and applications 208 (1997), pp. 141-157.

[17] Kato, Tosio \& Fujita, Hiroshi. On the nonstationary Navier-Stokes system. Rendiconti del Seminario Matematico della Università di Padova, 32 (1962), pp. 243-260.

[18] Leray, J. Sur le mouvement d'un liquide visquex emplissant l'espace. Acta Math. 63, 1934, pp. 193-248.

[19] Lions, Jacques L. Quelques méthodes de résolution des problémes aux limites non linéaires. Paris: Dunod, Gauthier-Villars, 1969.

[20] Liu, W. Asymptotic behavior of solutions of time-delayed Burger's equation. Discrete Continuous Dynamical Systems - B, 2 (2002), 47-56.

[21] Pazy, A. Semigroups of linear operators and applications to Partial Differential Equations. Applied mathematical Sciences, 44. Springer-Verlag, New York-Berlin, 1983.

[22] Planas, Gabriela \& Hernández, Eduardo. Asymptotic behaviour of two-dimensional time-delayed Navier-Stokes equations. Discrete and continuous dynamical systems. 21 (2008) no. 4, pp. 1245-1258.

[23] Rankin III, S. M. Semilinear evolution equations in Banach spaces with application to parabolic partial differential equations. Trans. Amer. Math. Soc. 336 (1993), no. 2, pp. 523-535.

[24] Robinson, James C. Infinite-Dimensional Dynamical Systems: An introduction to dissipative parabolic PDEs and the theory of global attractors. Cambridge texts in applied mathematics. Cambridge University Press. 2001. 
[25] Taniguchi, Takesi. The exponential behavior of Navier-Stokes equations with time delay external force. Discrete and Continuous Dynamical Systems. 12 (2005), no. 5, pp. 997-1018.

[26] Temam, Roger. Navier-Stokes equations and nonlinear functional analysis. CBMSNSF regional conference series in applied mathematics, no. 66, 1995.

[27] Temam, Roger. Navier-Stokes equations: Theory and numerical analysis. Studies in Mathematics and its applications. Volume 2. The Netherlands, 1984. 